\title{
Strongly coupled QFT dynamics via TQFT coupling
}

\author{
Mithat Ünsal \\ Department of Physics, North Carolina State University, \\ Raleigh, NC 2760\%, U.S.A. \\ E-mail: unsal.mithat@gmail.com
}

ABSTRACT: We consider a class of quantum field theories and quantum mechanics, which we couple to $\mathbb{Z}_{N}$ topological QFTs, in order to classify non-perturbative effects in the original theory. The $\mathbb{Z}_{N}$ TQFT structure arises naturally from turning on a classical background field for a $\mathbb{Z}_{N}$ 0- or 1-form global symmetry. In $\mathrm{SU}(N)$ Yang-Mills theory coupled to $\mathbb{Z}_{N}$ TQFT, the non-perturbative expansion parameter is $\exp \left[-S_{I} / N\right]=\exp \left[-8 \pi^{2} / g^{2} N\right]$ both in the semi-classical weak coupling domain and strong coupling domain, corresponding to a fractional topological charge configurations. To classify the non-perturbative effects in original SU $(N)$ theory, we must use $\operatorname{PSU}(N)$ bundle and lift configurations (critical points at infinity) for which there is no obstruction back to $\mathrm{SU}(N)$. These provide a refinement of instanton sums: integer topological charge, but crucially fractional action configurations contribute, providing a TQFT protected generalization of resurgent semi-classical expansion to strong coupling. Monopole-instantons (or fractional instantons) on $T^{3} \times S_{L}^{1}$ can be interpreted as tunneling events in the 't Hooft flux background in the PSU $(N)$ bundle. The construction provides a new perspective to the strong coupling regime of QFTs and resolves a number of old standing issues, especially, fixes the conflicts between the large- $N$ and instanton analysis. We derive the mass gap at $\theta=0$ and gaplessness at $\theta=\pi$ in $\mathbb{C P}^{1}$ model, and mass gap for arbitrary $\theta$ in $\mathbb{C P}^{N-1}, N \geq 3$ on $\mathbb{R}^{2}$.

Keywords: Confinement, Sigma Models, Solitons Monopoles and Instantons, Topological Field Theories

ARXIV EPRINT: 2007.03880 


\section{Contents}

1 Introduction $\mathbf{2}$

1.1 Earlier works on Yang-Mills on $T^{3} \times \mathbb{R}$ : not all QM reductions are the same. 5

2 Quantum mechanical $T_{N}$ and $\left(T_{N} / \mathbb{Z}_{N}\right)_{p}$ models 7

$\begin{array}{lll}2.1 \text { Coupling to } \mathbb{Z}_{N} \text { TQFT background } & 12\end{array}$

$\begin{array}{ll}2.2 \text { Uses of gauging } \mathbb{Z}_{N} & 15\end{array}$

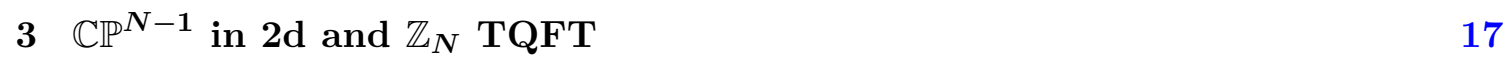

$\begin{array}{ll}3.1 \text { 't Hooft flux using } \frac{(\mathrm{SU}(N) \times \mathrm{U}(1))}{\mathbb{Z}_{N}} & 19\end{array}$

3.2 Fractional instantons in $\mathbb{C P}^{N-1}$ on large $M_{2} \quad 20$

3.3 Flavor twist and 't Hooft flux, and relation to $\mathbb{R} \times S^{1}$

3.4 Interpolation between weak and strong coupling holonomy potentials 25

$3.5 \mathcal{N}=(2,2) \mathbb{C} \mathbb{P}^{N-1}$ model on $\mathbb{R}^{2}$ with soft mass $\quad 27$

4 Continuation of $\mathbb{C P}^{N-1}$ semiclassics to strong coupling 30

4.1 Fateev, Frolov, Schwarz, and Berg, Lüscher, in retrospect 32

4.2 Incorporating $\theta$ and fractional anti-instantons into FFS-BL 35

4.3 Fermionization, Abelian rebosonization and mass gap at arbitrary $N \quad 38$

4.4 Fractional instanton renormalization group (FIRG) 42

4.5 Non-abelian bosonization: mass deformed $\mathrm{SU}(N)_{1}$ WZW 43

4.6 Secrets of Coleman's formula: relation of RG with all orders semi-classics 44

$4.7 \mathbb{C P}^{1}$ at $\theta=\pi \quad 46$

4.8 Why is this working? (Improving Polyakov's optimism with RG) 48

5 What are we summing over in semiclassics in $\mathrm{dYM}$ theory on $\mathbb{R}^{\mathbf{3}} \times S^{\mathbf{1}}$ ? 51

5.1 Mini-space formalism: global constraints in monopole-instanton sums 53

5.2 Vacuum energy density and multi-branch structure 56

6 Coupling Yang-Mills theory to $\mathbb{Z}_{N}$ TQFT $\quad 57$

6.1 $T^{3} \times S_{L}^{1}$ with 't Hooft flux and monopole-instantons $\quad 59$

6.2 Two types of monopole events, Born-Oppenheimer and $T_{N}$ QM 62

6.3 What does $\mathbb{Z}_{N}$ TQFT background has anything to do with monopoles? 63

6.4 Fractionalization in $\mathcal{N}=1 \mathrm{SU}(N)$ theory on $M_{4} \quad 67$

$\begin{array}{lll}7 & \text { Prospects and comments } & 68\end{array}$

A Yang-Mills with flux on $1^{3} \times S_{\beta}^{1}$ lattice vs. $T_{N}$ model $\quad 75$

$\begin{array}{ll}\text { B Aside: how to fix merons in } \mathbb{C P}^{1} \text { ? } & 77\end{array}$ 


\section{Introduction}

The applications of coupling a topological quantum field theory (TQFT) to quantum field theory (QFT) received recent interest in the discussion of mixed anomalies [1-3]. In this context, it is used to extract kinematic constraints imposed by symmetry on a QFT. This is an exact, albeit non-dynamical information about quantum theory.

Here, we would like to explore the implications of coupling a TQFT to QFT in the study of non-perturbative dynamics, a territory that we cannot expect to be exact, in search of reliable approximations. We want to take advantage of robustness of TQFT in parts of the story. One of our physical goals is to understand what controls the strength of nonperturbative effects in an asymptotically free QFT on large $d$-dimensional manifold $M_{d}$, where $M_{d}$ serves as a regularization for $\mathbb{R}^{d}$. More practically, we would like to understand the role of topological defects such as instantons, monopole instantons and fractional instantons in the dynamics much more precisely both in weak coupling semi-classical domain and in strong coupling domain by using the restrictions that follows from TQFT.

Let us denote a theory by $\mathrm{T}$ and its global symmetry by $G$. To be less abstract, we develop three (extremely) parallel stories. Quantum mechanics of a particle on a circle in the presence of a potential with $N$ degenerate minima, that we call $T_{N}$ model for short, $2 d \mathbb{C P}^{N-1}$ model, and $4 d \mathrm{SU}(N)$ Yang-Mills theory. $T_{N}$ quantum mechanics and YangMills theory possess a $\mathbb{Z}_{N}$ global symmetry, 0 -form or 1 -form, respectively. $\mathbb{C P}^{N-1}$ has a $\mathrm{SU}(N) / \mathbb{Z}_{N}$ global symmetry. One can turn on a classical background field for these symmetries (also called background gauging). ${ }^{1}$ In doing so, coupling QFT $T$ to a $\mathbb{Z}_{N}$ TQFT becomes strictly necessary, we call the resulting theory as $\mathrm{T} / \mathbb{Z}_{N}$ theory. ${ }^{2}$ As a result of this procedure, we learn the existence of new non-perturbative effects and scales relevant to the original theory, see figure 1.

A recurring theme can be described simply in quantum mechanics. Consider $T_{N}$ model, with $\mathbb{Z}_{N}$ shift symmetry, figure 2 . The partition function is described as a sum over periodic paths on $S^{1}$, it receives contribution only from integer winding number $W \in \mathbb{Z}$ configurations. Refer to the action of $W=1$ instanton as $S_{I}$. There are clearly fractional instantons $I_{j}$ with winding number $W \in \frac{1}{N} \mathbb{Z}$ and action $S_{I} / N$ in the theory, which contribute at leading order to the spectrum, but forbidden to contribute to partition function, because they are not periodic paths in $S^{1}$. Now, if we couple the theory to $\mathbb{Z}_{N}$ TQFT and gauge $\mathbb{Z}_{N}$, these configurations become closed paths in $S^{1} / \mathbb{Z}_{N}$, but there is an obstruction to lift them to closed paths in original $S^{1}$. Yet, a pair of loops with winding numbers $+1-1=0$

\footnotetext{
${ }^{1}$ We use background gauging for turning on a classical background field for a global symmetry and coupling it in a gauge invariant manner to the theory. Note that with a background field turned on, it is still meaningful to look at correlators that transform under background gauging. These are non-trivial and transform covariantly. There is no path integration at this stage. If we choose to sum over all possible backgrounds, i.e, perform a path integral over the background field by making it dynamical, we refer to it as gauging. In this case, the correlators that transform under gauge transformations are identically zero by Elitzur's theorem [4]. Both of these ideas are used throughout this work.

${ }^{2}$ Strictly speaking, $\mathrm{T} / \mathbb{Z}_{N}$ is appropriate description for QM and Yang-Mills. In $\mathbb{C P}^{N-1}$, it is slight abuse of language. To turn on $\mathrm{SU}(N) / \mathbb{Z}_{N}$ background, we first turn on an $\mathrm{SU}(N)$ background field, and then, couple the theory to a $\mathbb{Z}_{N}$ TQFT. This is what we mean by $\mathrm{T} / \mathbb{Z}_{N}$ model in $\mathbb{C P}^{N-1}$.
} 
Standard classification

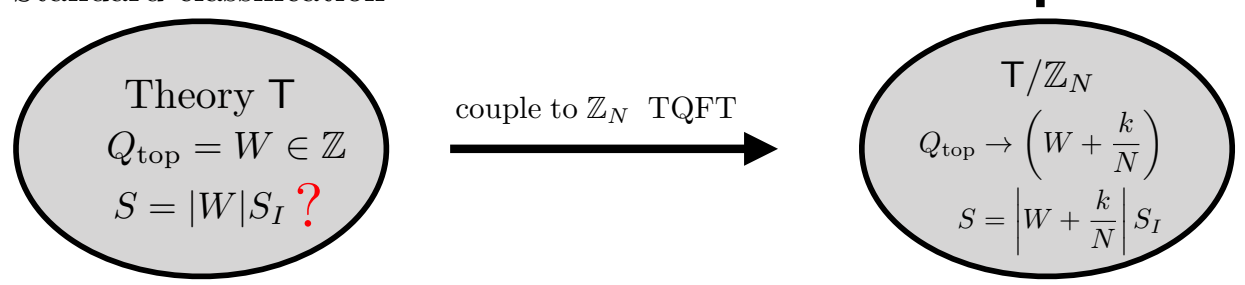

Figure 1. By coupling a QFT to a TQFT, the topological charge and action become fractional. Such configurations do not lift to the partition function of the theory $\mathrm{T}$, but their integer topological charge and fractional action composites do. Even the $W=\frac{1}{N}$ fractional instantons contribute to some observables in $\mathrm{T}$, but not to partition function. We demonstrate that the non-perturbative expansion parameter in theory is $\exp \left[-S_{I} / N\right]$ instead of $\exp \left[-S_{I}\right]$. This statement was already known to be true in semi-classical domain. The TQFT coupling tells us that it is also true in strong coupling domain.

in $S^{1} / \mathbb{Z}_{N}$ lift to $S^{1}$ as $\frac{1}{N}-\frac{1}{N}=0$ and contribute to the partition function with action $2 S_{I} / N$. The precise semi-classical description of this saddle here is critical point at infinity [5] which we explain in QM discussion. So, there are zero winding, and fractional action non-BPS configuration contributing to partition function in $T_{N}$ quantum mechanics.

We formalize this construction as follows in QM and QFT.

- In $d=1,2,4$, we describe a $\mathbb{Z}_{N}$ TQFT and couple it to the theory T: quantum mechanics, $\mathbb{C} \mathbb{P}^{N-1}$ and Yang-Mills, respectively.

- By turning on a classical $\mathbb{Z}_{N}$ background field or by gauging it (summing over all possible backgrounds, corresponding to $\mathrm{T} / \mathbb{Z}_{N}$ ), we find a set of saddles which do not lift to the original theory $\mathrm{T}$. But fractional action non-BPS composites of such objects lift to $\mathrm{T}$ and contribute to partition function.

- We can view the (fixed) classical $\mathbb{Z}_{N}$ TQFT backgrounds as (fixed) 't Hooft fluxes or twisted boundary conditions in $d=2,4[6,7]$.

- In Yang-Mills theory, the non-trivial configurations in the $\mathrm{SU}(N) / \mathbb{Z}_{N}$ bundle do not uplift to $\mathrm{SU}(N)$ bundle, but certain integer topological charge and fractional action configurations do. The same statement is also true in $\mathbb{C} \mathbb{P}^{N-1}$, where $\mathrm{U}(1) / \mathbb{Z}_{N}$ vs. 
$\mathrm{U}(1)$ are appropriate bundles. Both of these statements are independent of strength of coupling, in particular, valid at both weak and strong coupling due to TQFT coupling.

- Since in order to find the saddles in $T_{N}$ model, we need to directly look at either $T_{N}$ with classical $\mathbb{Z}_{N}$ background $\left(A^{(1)}, A^{(0)}\right)$ or equivalently, $\mathbb{Z}_{N}$ twisted boundary conditions or $\left(T_{N} / \mathbb{Z}_{N}\right)_{p}$ models where $\mathbb{Z}_{N}$ is gauged, we claim that in order to find the relevant saddles of $\mathrm{SU}(N)$ gauge theories, we should first consider either $\mathrm{SU}(N)$ with background gauge field $\left(B^{(2)}, B^{(1)}\right)$ for the $\mathbb{Z}_{N}^{[1]} 1$-form symmetry or $\left(\mathrm{SU}(N) / \mathbb{Z}_{N}\right)_{p}$ theory where $\mathbb{Z}_{N}^{[1]}$ is gauged. After finding these configurations, we can patch them up to find the ones that can be lifted to $\mathrm{SU}(N)$ theory. These are fractional action (e.g. $\left.2 S_{I} / N\right)$ configurations that contribute to the partition function of $\mathrm{SU}(N)$ theory.

- These configurations are TQFT-protected, and exist both at weak and strong coupling. In compactified theories, they exist both at $T_{\text {large }}^{d-1} \times S_{\text {small }}^{1}$ as well as $T_{\text {large }}^{d}$ where the latter is a place holder for $\mathbb{R}^{d}$.

- This construction shows that, in $d=2 \mathbb{C P}^{N-1}$ and $d=4$ Yang-Mills theory, the nonperturbative effects are not controlled by the (BPST) instanton factor $\mathrm{e}^{-S_{I}+\mathrm{i} \theta}[8,9]$, but instead by exponentially larger $\mathrm{e}^{-S_{I} / N+\mathrm{i} \theta / N}$ fractional instanton factor, due to TQFT-protection.

- This extends our earlier result in flavor $\Omega_{F}$-twisted $\mathbb{C P}^{N-1}$ model on semi-classical small $\mathbb{R} \times S_{L}^{1}[10,11]$ to large $\mathbb{R} \times S_{L}^{1}$, to the strong coupling domain, by augmenting flavor twisted theory with a $\mathbb{Z}_{N}$ TQFT background (i.e. with 't Hooft twist.) It also extends some results in semi-classical deformed Yang-Mills theory, $\mathcal{N}=1 \mathrm{SYM}$, $\mathrm{QCD}$ (adj) and some other QCD-like theories (see [12-15]) from small $\mathbb{R}^{3} \times S_{L}^{1}$ to large $\mathbb{R}^{3} \times S_{L}^{1}$, to the strong coupling domain by coupling these systems to $\mathbb{Z}_{N}$ TQFTs.

- Based on matching weak coupling semi-classical descriptions, matching of global symmetries and mixed 't Hooft anomalies, and improving an old work of Fateev, Frolov, Schwarz, and Berg, Lüscher $[16,17]$ in crucial ways, we show that low-energy spectrum of the $\mathbb{C P}^{N-1}$ model is described by $N$-flavor massive Schwinger model or its non-abelian bosonization, the mass deformation of $\mathrm{SU}(N)_{1}$ WZW model with an extra scalar.

- The mass parameter in Schwinger model, or mass deformed WZW models, due to matchings of semi-classical descriptions, map to a fractional instanton effect $\mathrm{e}^{-S_{I} / N}$ in $\mathbb{C P}^{N-1}$, which is nothing but the strong scale of the theory. By studying renormalization group for topological defect operators [18] (derived originally to explain the Kosterlitz-Thouless phase transition [19]), we describe the low energy spectrum of the theory.

- We determine the mass gap and low energy spectrum for arbitrary $\theta$ in $\mathbb{C P}^{N-1}$ on $\mathbb{R}^{2}$ via this procedure. For $N \geq 3$, the spectrum is gapped for any $\theta$, and composed of an adjoint and singlet. For the $N=2$ theory, the theory is gapped at $\theta \neq \pi$. We 
show the existence of a gapless triplet at $\theta=\pi$. Our formalism give a prediction for the mass gap in the $\mathbb{C P}^{1}$ model as $m_{\text {gap }}(\theta)=\Lambda\left|\cos \frac{\theta}{2}\right|^{2 / 3}$, which we hope can be tested by other means.

Our construction resolves many puzzles concerning conflicts between large- $N$ vs. instantons, instantons vs. multi-branch structure of vacua, incorrect $\theta$-angle dependence of vacuum energy due to instantons, instantons vs. $\eta^{\prime}$ puzzle, and many others mentioned in books and reviews [20-23]. In retrospect, our current construction proves why recent studies on $\mathbb{R}^{d-1} \times S^{1}$ in the context of adiabatic continuity and resurgent semi-classical analysis $[10,11,14,15]$ capture interesting non-perturbative dynamics of QFT on $\mathbb{R}^{d}$. In the light of our construction, it may be worthwhile to revisit the meron idea of Gross et al. [24-26] on $\mathbb{R}^{d}$. This idea requires some fixing which can easily be done, for the $N=2$ case, see section B. It also needs correct generalization to $N \geq 3$.

\subsection{Earlier works on Yang-Mills on $T^{3} \times \mathbb{R}$ : not all QM reductions are the same.}

We finalize the introduction by describing historical precedents to this work, ${ }^{3}$ especially concerning the different class of constructions in a quantum mechanical reduction on $T^{3} \times \mathbb{R}$. This will also give us an opportunity to highlight where our work resembles or differs from the earlier works.

An exact solution to self-duality equation with topological charge $Q=1 / N$ and action $\frac{8 \pi^{2}}{g^{2} N}$ on $T^{4}$ is first found by 't Hooft [27], and generalized by van Baal [28]. 't Hooft original physical motivation was to find configurations which survive in the large- $N$ limit, and which are exponentially more important than the usual instantons even at finite- $N$. (The usual instantons are suppressed as $e^{-N}$ in the large- $N$ limit, and are irrelevant.) These solutions can be interpreted as configurations in $\mathrm{SU}(N)$ with discrete flux background turned on, or in $\operatorname{PSU}(N)$ bundle. However, these are constant solutions, and the geometry of $T^{4}$ must satisfy a certain aspect ratio to have a $\frac{8 \pi^{2}}{g^{2} N}$ action. Unlike on $\mathbb{R}^{3} \times S^{1}$ where it is easy to find non-trivial (space-time dependent) fractional instanton solutions, the monopole-instantons, where dynamics abelianize at long distances due to adjoint-Higgsing sourced by the vev of gauge holonomy, it proved to be extremely difficult to find non-trivial solutions in systems which do not abelianize at long distance.

Discrete flux background and twisted boundary conditions are used to reduce $\mathcal{N}=1$ $\mathrm{SU}(N)$ SYM to quantum mechanics on $T^{3} \times \mathbb{R}$ and employed to calculate supersymmetric index in Witten's work [29]. The work closest in spirit to our construction, working with non-supersymmetric theories, and aiming to preserve the vacuum structure and fractional instantons in a quantum mechanical limit, are the works of Gonzalez-Arroyo et al. In their construction, dynamics do not abelianize, and they have found by numerical simulations on latticized $T^{3} \times \mathbb{R}$ that time-dependent fractional instanton solutions with action $\frac{8 \pi^{2}}{g^{2} N}$ exist in the presence of 't Hooft flux [30-38]. The analytic form of these solutions is

\footnotetext{
${ }^{3}$ We thank anonymous referee for encouraging us to write this part which clarifies the relation between the present work and earlier discussions of Yang-Mills theory in a quantum mechanical reduction down to small $T^{3} \times \mathbb{R}$.
} 
still an open and important problem. The difference between the works of Witten and Gonzalez-Arroyo, and our work is following. We reduce deformed YM on $\mathbb{R}^{3} \times S_{\beta}^{1}$ (which is adiabatically connected to pure YM on $\mathbb{R}^{4}$ ) down to $T^{3} \times S_{\beta}^{1}$ by using magnetic GNO flux in the co-weight lattice of $\mathrm{SU}(N)$. This is more refined data than discrete 't Hooft flux. Magnetic $N$-ality determines the discrete magnetic flux. The fact that we can turn on $\boldsymbol{\mu}_{1}$ GNO flux and this is degenerate in energy with $\boldsymbol{\mu}_{\mathbf{1}}-\boldsymbol{\alpha}_{\mathbf{1}}, \ldots, \boldsymbol{\mu}_{1}-\sum_{j=1}^{N-1} \boldsymbol{\alpha}_{j}$ (all with discrete magnetic flux one) helps us to identify the tunneling in QM with the monopoleinstantons on $\mathbb{R}^{3} \times S_{\beta}^{1}$, which carry magnetic charges in the extended simple root system of $\mathrm{SU}(N)$. In both construction, there are $N$-classical vacua (sourced by turning on a classical background) and $N$ - fractional instantons with topological charge $Q=1 / N$ and action $\frac{8 \pi^{2}}{g^{2} N}$, an extremely desirable aspect.

A frequently asked question is the relation between Luscher [39] and van Baal's works [40, 41], which employs $T^{3} \times \mathbb{R}$, without incorporating discrete flux, and our present work. ${ }^{4}$ These works would like to benefit from the absence of phase transitions in finite volume. However, unlike our construction, in these compactification, vacuum structure is not persistent. In particular, in $\mathrm{SU}(N)$ theory, there are $N^{3}$ vacua related to perturbative breaking of $\left(\mathbb{Z}_{N}^{[0]}\right)^{3}$ center symmetry to all orders in perturbation theory, instead of $N$ classical vacua that emerge from classical flux insertion. However, 0 -form symmetries cannot break spontaneously in QM unless enforced by a mixed anomaly. ${ }^{5}$ Indeed, there are instantons in the theory leading to a unique vacuum. These instantons between $N^{3}$ perturbative vacua are not classical fractional instantons with action $\frac{8 \pi^{2}}{g^{2} N}$ or instantons with action $\frac{8 \pi^{2}}{g^{2}}$. Rather, these instantons arise from balancing of the classical action with one-loop potential for the gauge holonomy, i.e, they are "quantum instantons", with action of order $O(1 / g)$, i.e, they are not related to fractional instantons that we are talking about, that exist in thermo-dynamic limit on $\mathbb{R}^{3} \times S^{1}$ or the ones that appear in $\operatorname{PSU}(N)$ bundle classification.

Furthermore, if we consider the large- $N$ limit of the Luscher [39] and van Baal's compactification [40, 41], the tunneling amplitude between $N^{3}$ perturbative vacua has an $N$ dependence of the form $e^{-\sqrt{N}}$, and the tunneling becomes forbidden in the $N \rightarrow \infty$ limit. That is, even in finite volume, there is a phase transition in the large- $N$ limit (which is also a thermo-dynamic limit) of their construction, between the small $T^{3} \times \mathbb{R}$ theory and large $T^{3} \times \mathbb{R}$ theory according to $\left(\mathbb{Z}_{N}^{[0]}\right)^{3}$ center-symmetry realization. (This phase transition is actually the reason why Eguchi-Kawai reduction [42] in its original form without discrete flux fails $[43,44]$.) In sharp contrast, in our and Gonzalez-Arroyo's constructions, there are $N$-classical vacua, induced by classical background, and the tunneling between them is a classical fractional instanton effect with action $\frac{8 \pi^{2}}{g^{2} N}$ and does not disappear in the large- $N$ limit. (This is actually the reason why Twisted-Eguchi-Kawai reduction with judiciously chosen discrete flux satisfies large- $N$ volume independence [45].) Very importantly, in our set-up, the fractional instantons induce effects such as $\exp \left[-\frac{8 \pi^{2}}{g^{2} N}+i \frac{\theta}{N}\right]$. One of the most

\footnotetext{
${ }^{4}$ van Baal also have important works on the discrete flux backgrounds and classification of the configurations in $\operatorname{PSU}(N)$ bundle.

${ }^{5}$ In fact, $d-1$ form symmetries cannot break spontaneously in $d$ dimensions unless enforced by a mixed anomaly.
} 
interesting non-perturbative phenomena in infinite volume gauge theory is that observables are $N$-branched functions, where each branch is $2 \pi N$ periodic, yet, the observables are $2 \pi$ periodic, with cusps at $\theta=\pi[46]$. The set of fractional instantons (including critical points at infinity) are compatible with a complete Fourier basis, which can describe such non-trivial observables with multi-branched structure, and this compatibility of saddles with a complete basis for Fourier expansion of observables is far from trivial. Indeed, we have the same intentions with Luscher and van Baal, but thanks to the GNO and discrete flux backgrounds we use, our quantum mechanical constructions have a remembrance of fractional topological configurations on $\mathbb{R}^{3} \times S^{1}$ and the configurations in $\operatorname{PSU}(N)$ bundle even on large- $T^{4}$. Therefore, the constructions with flux are more powerful strategies to understand non-perturbative Yang-Mills dynamics across different length scales.

Finally, perhaps, a point that is not sufficiently understood (or emphasized) in the past, is the fact that the flux backgrounds have a re-interpretation as coupling the QFTs to TQFT (backgrounds) [2]. These theories are globally different, but locally the same. For example, mass spectrum are completely identical on large $T^{4}$. The TQFT coupling instructs us that the existence of fractional topological charge configurations is independent of $T^{4}$ size, and is valid at both weak and strong coupling. This is explored in detail in present work and in a companion paper [47].

\section{Quantum mechanical $T_{N}$ and $\left(T_{N} / \mathbb{Z}_{N}\right)_{p}$ models}

Consider quantum mechanics of a particle on a circle with a potential of the form

$$
V(q)=-\cos (N q), \quad q \sim q+2 \pi
$$

$q$ is physically identified with $q+2 \pi$. The theory has a $\mathbb{Z}_{N}$ global shift symmetry,

$$
\mathbb{Z}_{N}: q \mapsto q+\frac{2 \pi}{N}
$$

The system has $N$-degenerate minima to all orders in perturbation theory. The partition function, $Z(\beta)=\operatorname{tr}[\exp (-\beta H)]$, in the $\beta \rightarrow \infty$ limit, is dominated by the lowest- $N$ states. Within the Born-Oppenheimer approximation, one can forget about higher states in the spectrum, as non-perturbative instanton induced splittings are exponentially smaller than splittings between harmonic oscillator levels. In this limit, the system may be viewed as $N$-site lattice model with nearest neighbor hopping interaction, described by a tight-

binding approximation. For convenience, we call this system $T_{N}$ model. See figure 2. Some semi-classical and anomaly aspects of this model are studied in [3, 48, 49].

This simple system provides a playground for some ideas that we will employ in diverse non-trivial QFTs. Hence, we explore in detail the connection between various ways of looking to this system and interpret them in simple physical terms. The connection between the $\mathbb{Z}_{N}$ twisted boundary conditions, and turning on $\mathbb{Z}_{N}$ background field is well known. These constructions have a precise interpretation as coupling QM to $\mathbb{Z}_{N}$ TQFT, which is fairly useful in QFT. Later, we may also choose to gauge $\mathbb{Z}_{N}$ symmetry. This is equivalent to summing over all possible background fields or twisted boundary conditions, or performing 


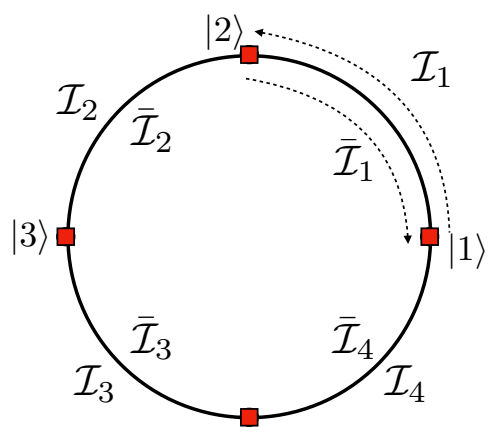

$|4\rangle$

Figure 2. $T_{N}$ model is quantum mechanics with periodic potential $-\cos (N q)$ on a circle with fundamental domain $q \in[0,2 \pi]$. In the Born-Oppenheimer approximation, it is equivalent to $N$ site lattice model. In partition function, we sum only over periodic paths, corresponding to integer winding number topologies, but obviously, there are other saddles with fractional winding number.

a path integral over the TQFT. Gauging, of course, changes the theory, from $T_{N}$ to $T_{N} / \mathbb{Z}_{N}$. In the latter, $q$ and $q+\frac{2 \pi}{N}$ are physically identified. i.e, we end up with the particle on a circle with 1-minimum on the circle. However, there are different versions of this 1-site theory that we call $\left(T_{N} / \mathbb{Z}_{N}\right)_{p}$, depending how we gauge $\mathbb{Z}_{N}$. The $p$ in the $\left(T_{N} / \mathbb{Z}_{N}\right)_{p}$ has three equivalent, but somehow different sounding interpretations, as discrete theta angle $\theta_{p}$, or level $p$ Chern-Simons term that is present in TQFT, or picking Bloch state with quasi-momentum $p$ from the Hilbert space of the original theory, which we describe in detail, as it translates verbatim to QFT.

Basics. The path-integral representation of the partition function is

$$
\begin{aligned}
& Z(\beta)=\operatorname{tr}\left[\mathrm{e}^{-\beta H}\right]=\int_{q(\beta)=q(0)} \mathcal{D} q \exp (-S[q]), \quad \text { where } \\
& S[q]=\frac{1}{g} \int d \tau\left(\frac{1}{2} \dot{q}^{2}+V(q)\right)+\frac{\mathrm{i} \theta}{2 \pi} \int \mathrm{d} q
\end{aligned}
$$

The trace $\operatorname{tr}[\cdot]$ in the operator formalism translates in the path integral formalism to integration over all paths obeying the periodic boundary condition, $q(\beta)=q(0)$. The periodic paths are classified according to the homotopy group of the maps

$$
q(\tau): S_{\beta}^{1} \rightarrow S^{1}, \quad \pi_{1}\left(S^{1}\right)=\mathbb{Z}
$$

which is nothing but winding number (corresponding to topological charge):

$$
W=\frac{1}{2 \pi} \int \mathrm{d} q \in \mathbb{Z}
$$

which is integer valued.

Despite the fact that the partition function is given by summing over integer winding number $W \in \mathbb{Z}$ configurations, it is clear that there are classical solutions with fractional 
topological charge, called fractional instantons, see figure $2 .{ }^{6}$ The configuration with $W=1$ is an instanton, and its action is $S_{W=1} \equiv S_{I}$. This is our yard-stick throughout the problem. The configurations with $W=1 / N$ are referred to as fractional instantons, and their action is $S_{I} / N$. These are tunneling configurations connecting neighboring minima $j \rightarrow j+1$. Clearly, a single fractional instanton $\mathcal{I}_{j}$ cannot contribute to the partition function, as it does not obey the periodic boundary condition in (2.3). Equivalently, these are periodic paths in $S^{1} / \mathbb{Z}_{N}$ which cannot be lifted to $S^{1}$. However, an $\mathcal{I}_{j}, \overline{\mathcal{I}}_{j}$ pair, which has $W=0$, but action $2 S_{I} / N$, must contribute to the partition function since it is a periodic path in $S^{1}$.

Digression on critical points at infinity and resurgent cancellations. The $\mathcal{I}_{j}, \overline{\mathcal{I}}_{j}$ configurations can be viewed as critical points at infinity. Here, we will go over this concept fairly quickly. See [5] for details and [10] for broad review. The fact that the combination of $\mathcal{I}_{j}$ and $\overline{\mathcal{I}}_{j}$ configuration is often called "unstable" in old literature is a red-herring. In semi-classics, the critical point at infinity has a non-Gaussian Lefschetz thimble (steepest descent cycle) that one must integrate over. This contribution maps to second order terms in cluster expansion as

$$
\frac{\beta^{2}}{2 !}\left[\mathcal{I}_{j}\right]\left[\overline{\mathcal{I}}_{j}\right]+\frac{\beta}{1 !}\left[\mathcal{I}_{j} \overline{\mathcal{I}}_{j}\right]_{ \pm}
$$

where the first, maximally extensive term in $\beta$ is dilute (non-interacting) instanton gas contribution and sub-extensive term capture the correlated instanton-anti-instanton event $\left[\mathcal{I}_{a} \overline{\mathcal{I}}_{a}\right]_{ \pm}$which is twofold ambiguous. Remarkable fact that comes from resurgent analysis is that this ambiguity cancels against the ambiguity of lateral Borel resummation of perturbation theory for vacuum energy, $\mathbb{B} E_{0, \pm}$. At second order in semi-classics, we find the cancellation, and

$$
\operatorname{ImB} E_{0, \pm}+\operatorname{Im}\left[\mathcal{I}_{j} \overline{\mathcal{I}}_{j}\right]_{ \pm}=0
$$

The combination is unambiguous and meaningful within resurgent semi-classical analysis framework [10].

Strictly speaking, action $2 S_{I} / N$ and $W=0$ configurations can equivalently be viewed as uncorrelated events $\left[\mathcal{I}_{j}\right]$ and $\left[\overline{\mathcal{I}}_{j}\right]$ and correlated bion events $\left[\mathcal{I}_{j} \overline{\mathcal{I}}_{j}\right]_{ \pm}$, for which its real unambiguous part $\operatorname{Re}\left[\mathcal{I}_{j} \overline{\mathcal{I}}_{j}\right]$ contribute to the partition function, as both correspond to periodic paths in $S^{1} .^{7}$ Similarly, configuration such as an ordered product $\prod_{j=1}^{N} \mathcal{I}_{j}=$ $\mathcal{I}_{1} \mathcal{I}_{2} \ldots \mathcal{I}_{N}$ with integer topological charge $W=1$, and integer action $1 \times S_{I}$ must contribute to the partition function since it is also a periodic path. More generally, generically nonBPS configurations with action $\left(W+\frac{2 \bar{n}}{N}\right) S_{I}$ and topological charge $W \in \mathbb{Z}$ contribute to partition function.

\footnotetext{
${ }^{6}$ These are obvious in quantum mechanics, but not always so obvious in QFT. This is the reason why we lay out a somewhat abstract formalism in QM, which carries over to QFT. Obvious facts in QM will map to non-obvious statements in QFT both at weak and strong coupling on $d$-manifolds, $M_{d}$ including $\mathbb{R}^{d}$ limits.

${ }^{7}$ Throughout the paper, we will not bother much with the resurgent structure, but it is often at the back of our minds. It is important that most of the critical points that contribute to actions in QFTs and QM are "critical points at infinity", and is decomposable to BPS and non-BPS configurations. We encourage the reader to go over [5] if necessary. This background knowledge allows us to take some steps swiftly in the course of this work.
} 
Let us now derive the fractional instanton decomposition of the path integral. Since we already know the energy spectrum, we can take a short cut and use it to derive the fractional instanton sum and see explicitly the paths that contribute at leading semi-classics.

Within the Born-Oppenheimer approximation, we can write a tight-binding Hamiltonian to describe low energy physics (We shifted zero point energy $\hbar \omega / 2$ to zero, and $\xi=K \mathrm{e}^{-S / N}$ is the non-perturbatively small hopping parameter.)

$$
H=-\sum_{j=1}^{N} \xi \mathrm{e}^{i \theta / N}|j+1\rangle\langle j|+\text { h.c. }
$$

The energy spectrum is given by diagonalizing the Hamiltonian:

$$
E_{k}(\theta)=-2 K \mathrm{e}^{-S / N} \cos \frac{\theta+2 \pi k}{N}
$$

The ground state depends on what the theta angle is. The ground state energy is $E_{\mathrm{G} . S .}(\theta)=$ $\min _{k} E_{k}(\theta)$, exhibiting a two-fold degeneracy at odd-integer multiples of $\theta=\pi$.

Let us now turn the partition function into a fractional instanton sum:

$$
\begin{aligned}
Z(\beta, \theta) & =\sum_{k=0}^{N-1} \mathrm{e}^{2 \beta K \mathrm{e}^{-\frac{S}{N}} \cos \frac{\theta+2 \pi k}{N}} \\
& =\sum_{k=0}^{N-1} \sum_{n=0}^{\infty} \sum_{\bar{n}=0}^{\infty} \frac{1}{n !} \frac{1}{\bar{n} !}\left(\beta K \mathrm{e}^{-\frac{S}{N}+\mathrm{i} \frac{\theta+2 \pi k}{N}}\right)^{n}\left(\beta K \mathrm{e}^{-\frac{S}{N}-\mathrm{i} \frac{\theta+2 \pi k}{N}}\right)^{\bar{n}}
\end{aligned}
$$

We can perform the summation over $k$ by using the identity

$$
\sum_{k=0}^{N-1} \mathrm{e}^{\mathrm{i} 2 \pi k(n-\bar{n}) / N}=N \sum_{W \in \mathbb{Z}} \delta_{n-\bar{n}-W N}
$$

converting the sum over $k$, which takes place in the space of representations of $\mathbb{Z}_{N}$ translation symmetry into a sum over $W \in \mathbb{Z}$ which we will interpret as winding number. This gives the path integral in the leading order of dilute gas approximation.

$$
Z(\beta, \theta)=N \sum_{W \in \mathbb{Z}} \sum_{n=0}^{\infty} \sum_{\bar{n}=0}^{\infty} \frac{1}{n !} \frac{1}{\bar{n} !}\left(\beta K \mathrm{e}^{-S / N+\mathrm{i} \theta / N}\right)^{n}\left(\beta K \mathrm{e}^{-S / N-\mathrm{i} \theta / N}\right)^{\bar{n}} \delta_{n-\bar{n}-W N, 0}
$$

where delta function gives a constraint on the fractional instanton sum. Eq. (2.12) can be interpreted as a grand canonical ensemble of fractional instantons. For a term in the sum with $n$ fractional instanton, and $\bar{n}$ fractional anti-instantons, the periodic orbits correspond to:

$$
n-\bar{n}-W N=0, \quad \text { i.e., } n-\bar{n}=0 \bmod N
$$

These configurations which contribute to $Z(\beta, \theta)$ comes with fugacity

$$
\mathrm{e}^{-\frac{S_{I}}{N}(n+\bar{n})} \mathrm{e}^{\mathrm{i} \frac{\theta}{N}(n-\bar{n})}=\mathrm{e}^{-\left(W+\frac{2 \bar{n}}{N}\right) S_{I}} \mathrm{e}^{\mathrm{i} W \theta}
$$

i.e, they possess fractional action, but integer topological charge. 


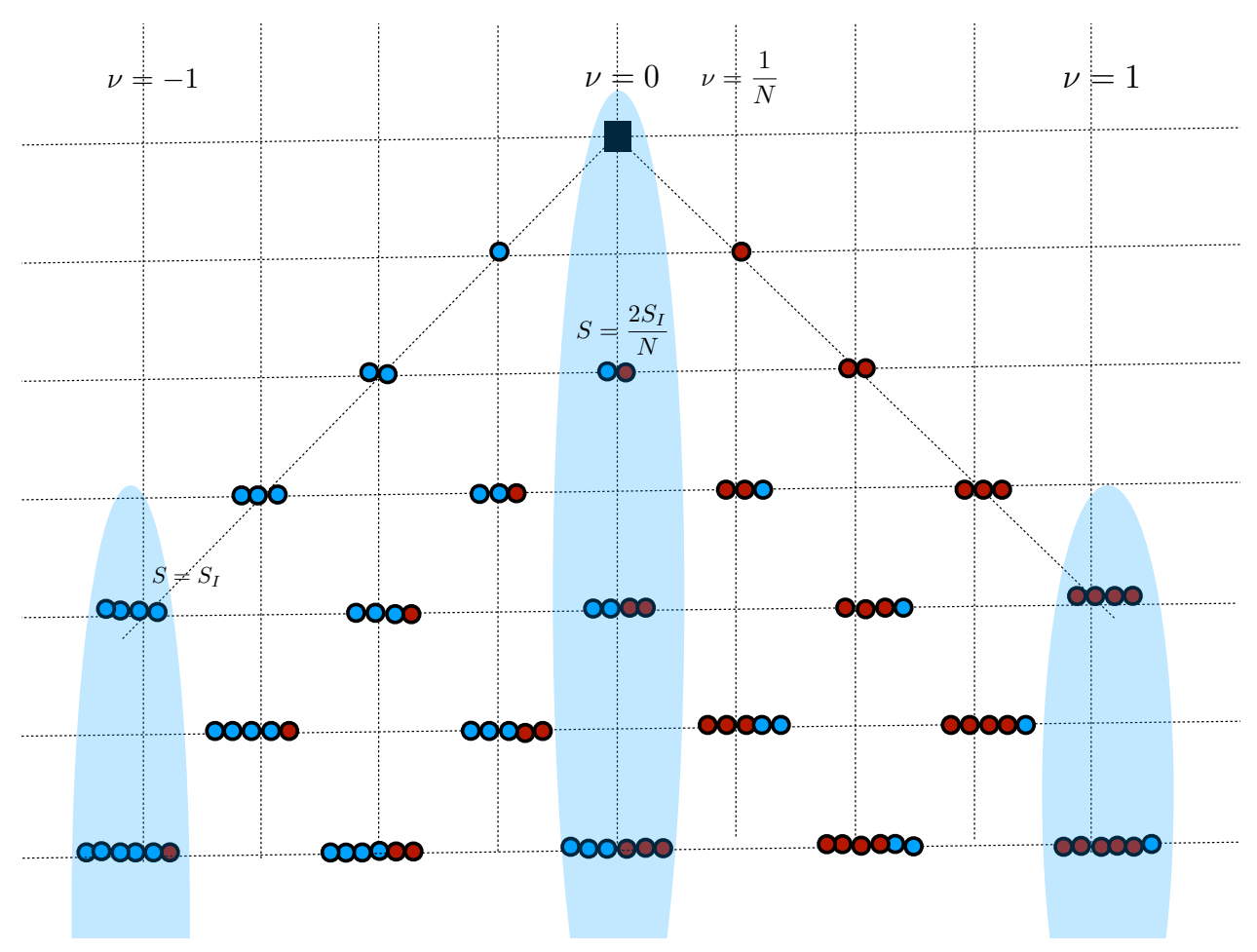

Figure 3. Only integer winding (topological charge) configurations corresponding to periodic paths contribute to partition function. To detect fractional instantons in QM is visually trivial (See figure 1). Formally, it requires either twisted boundary condition by using $\mathbb{Z}_{N}$ shift symmetry, or coupling to $\mathbb{Z}_{N}$ topological gauge theory. The latter more abstract formalism is more useful in gauge theories in four-dimensions, to understand the true nature of saddles contributing to YangMills theory. Despite the fact that $W=\frac{1}{N}, S=S_{I} / N$ fractional topological charge and fractional configurations are not contributing to partition function, $W=0, S=2 S_{I} / N$ fractional action configurations do. This is responsible for the emergence of multi-branch structure as a function of theta angle. This construction tells us that trying to understand the saddles in $\mathrm{SU}(N)$ gauge theory by working directly with $\mathrm{SU}(N)$ bundle is not correct. It neglects most important contributions.

The simple observation, that saddles with fractional action, but integer topological charge contributes to the path integral (see figure 3), is at the heart of all reliable semiclassical analysis of gauge theories, and sigma models, such as $\mathcal{N}=1 \mathrm{SYM}$, Yang-Mills with double-trace deformations, and $\mathbb{C} P^{N-1}$ models, and is responsible for the multi-branched structure of the vacuum energy. This structure naturally arise in the context of resurgence and sometimes called graded resurgence triangle [11, 50].

In (2.12), $K$ is the instanton prefactor that is not important for this particular discussion, and we will ignore it throughout the paper. $\beta$ is there due to position zero mode of a single fractional instanton. $\frac{\beta^{n}}{n !}$ arises due to the integration over the positions of $n$ fractional instantons, see e.g. [20]. The overall factor $N$ is due to $N$ distinct classical minima in the $q \in[0,2 \pi)$ fundamental domain. 
The constrained sum can be simplified further. Solving the constraint,

$$
\begin{aligned}
Z(\beta, \theta) & =N \sum_{W \in \mathbb{Z}}[\sum_{\bar{n}=0}^{\infty} \underbrace{\frac{\left(\beta K \mathrm{e}^{-\frac{S_{I}}{N}}\right)^{\bar{n}}}{\bar{n} !}}_{\bar{n} \text {-fractional anti-inst. }} \underbrace{\frac{\left(\beta K \mathrm{e}^{-\frac{S_{I}}{N}}\right)^{\bar{n}+W N}}{(\bar{n}+W N) !}}_{(\bar{n}+N) \text {-fractional inst. }}] \mathrm{e}^{\mathrm{i} W \theta} \\
& =N \sum_{W \in \mathbb{Z}}\left[I_{N W}\left(2 \beta K \mathrm{e}^{-\frac{S_{I}}{N}}\right)\right] \mathrm{e}^{\mathrm{i} W \theta}
\end{aligned}
$$

where $I_{N W}$ is the modified Bessel function of order $N W$. We learn that the series expansion of the modified Bessel function is actually an instanton sum. Since $Z(\theta+2 \pi)=Z(\theta),(2.15)$ is nothing but the Fourier series expansion of the partition function:

$$
Z(\theta)=\sum_{W \in \mathbb{Z}} Z_{W} \mathrm{e}^{\mathrm{i} W \theta}, \quad Z_{W}=N I_{N W}\left(2 \beta K \mathrm{e}^{-\frac{S_{I}}{N}}\right)
$$

Despite the fact that only periodic orbits with the theta angle dependence $e^{\mathrm{i} W \theta}$ contribute, the non-trivial theta-angle dependence which leads to multi-branched structure and $\theta$ dependence in the observables in the form $\frac{\theta+2 \pi k}{N}$ naturally arises! This fact will also help us in gauge theories on $\mathbb{R}^{4}$ and $\mathbb{R}^{3} \times S^{1}$.

\subsection{Coupling to $\mathbb{Z}_{N}$ TQFT background}

The quantum mechanical model we are studying has a $\mathbb{Z}_{N}$ global 0-form shift symmetry. First, we describe coupling it to a $\mathbb{Z}_{N}$ background gauge field. This stage will help us to reveal the existence of fractional instantons that actually exist in the $T_{N}$ model, in a more abstract way. But this somehow abstract formalism help us greatly in the QFT applications. We can later choose to sum over all possible backgrounds, which is equivalent to gauging $\mathbb{Z}_{N}$ completely, reducing the model to $T_{N} / \mathbb{Z}_{N}$, which is a 1 -site model. In our discussion, we aim to make the details fairly explicit and conceptual. This will provide important insights that we will use in QFT on $d$-manifolds $M_{d}$.

Coupling QM to TQFT. We first couple our quantum mechanical model to a classical $\mathbb{Z}_{N}$ topological gauge theory background $[1,2]$. The quantum topological theory is given by the partition function

$$
Z_{\text {top }, p}=\int \mathcal{D} A^{(1)} \mathcal{D} A^{(0)} \mathcal{D} F^{(0)} \mathrm{e}^{\mathrm{i} \int F^{(0)} \wedge\left(N A^{(1)}-\mathrm{d} A^{(0)}\right)+\mathrm{i} p \int A^{(1)}}
$$

Here, $F^{(0)}$ is a Lagrange multiplier zero-form field that we integrate over, and $\operatorname{ip} \int A^{(1)}$ term is Chern-Simons term in $1 d$, and $p$ must be an integer for the theory to be gauge invariant. $^{8}$

Integrating $F^{(0)}$, we end up with a pair, $\left(A^{(1)}, A^{(0)}\right)$ which obeys

$$
N A^{(1)}=\mathrm{d} A^{(0)}, \quad N \int A^{(1)}=\int \mathrm{d} A^{(0)}=2 \pi \mathbb{Z}
$$

\footnotetext{
${ }^{8}$ This term will provide different possibilities in gauging. It will turn out to be related to discrete theta angle, which in turn, determines which Bloch state is picked from the lowest $N$ state after gauging. For now, $p$ term is not very important and can be set to zero if desired.
} 
and is properly quantized. The path integration of TQFT is fairly trivial, and yields:

$$
\begin{aligned}
Z_{\mathrm{top}, p} & =\int \mathcal{D} A^{(1)} \mathcal{D} A^{(0)} \delta\left(\left(N A^{(1)}-\mathrm{d} A^{(0)}\right) \mathrm{e}^{\mathrm{i} p \int A^{(1)}}\right. \\
& =\frac{1}{N} \int \mathcal{D} A^{(0)} \mathrm{e}^{\mathrm{i} \frac{p}{N} \int d A^{(0)}} \\
& =\frac{1}{N} \sum_{k=0}^{N-1} \mathrm{e}^{\mathrm{i} \frac{2 \pi p k}{N}}=\delta_{p=0 \bmod N}
\end{aligned}
$$

$\left(A^{(1)}, A^{(0)}\right)$ pair describe a $\mathbb{Z}_{N}$ gauge field that can be turned on in quantum mechanical $T_{N}$ model to probe saddles, in particular, to probe the fractional instantons.

The 0 -form gauge invariance of the action (2.17) is given by

$$
A^{(1)} \mapsto A^{(1)}+\mathrm{d} \lambda^{(0)}, \quad A^{(0)} \mapsto A^{(0)}+N \lambda^{(0)}, \quad F^{(0)} \mapsto F^{(0)}
$$

To couple a classical $\mathbb{Z}_{N}$ background field to the $q$-field, we declare

$$
q \mapsto q-\lambda^{(0)},
$$

Gauge invariant combinations of our quantum field $a$ and classical fields $\left(A^{(1)}, A^{(0)}\right)$ are:

$$
N q+A^{(0)}, \quad d q+A^{(1)}=\left(\dot{q}+A_{\tau}\right) \mathrm{d} \tau
$$

Hence, the partition function in the classical $\left(A^{(1)}, A^{(0)}\right)$ background can be found by promoting $d q$ and $q$ to these gauge invariant combinations:

$$
\begin{aligned}
Z\left[\left(A^{(1)}, A^{(0)}\right), p\right]= & \int \mathcal{D} F^{(0)} \int_{q(\beta)=q(0)} \mathcal{D} q \mathrm{e}^{\mathrm{i} \int F^{(0)} \wedge\left(N A^{(1)}-\mathrm{d} A^{(0)}\right)+\mathrm{i} p \int A^{(1)}} \\
& \times \exp \left(-\frac{1}{g} \int d \tau\left(\frac{1}{2}\left(\dot{q}+A_{\tau}\right)^{2}-\cos \left(N q+A^{(0)}\right)\right)+\frac{\mathrm{i} \theta}{2 \pi} \int\left(\mathrm{d} q+A^{(1)}\right)\right)
\end{aligned}
$$

Note that at this stage, we do not perform a path integral over $\left(A^{(1)}, A^{(0)}\right)$. These are truly classical fields that we turn on according to what we want to do. Moreover, the path integral $\int \mathcal{D} q$ is over periodic paths.

Equivalence of symmetry twisted boundary condition to coupling QM to TQFT. The $\mathbb{Z}_{N}$ background gauge field is equivalent to the twisted boundary condition in path integral. Let us show this explicitly. Let $U$ denote the translation operator for $\mathbb{Z}_{N}$ shift symmetry in the $T_{N}$ model. Then, the sum over transition amplitude in quantum mechanics for sites $\ell$ units apart is given by:

$$
Z_{\ell}=\operatorname{tr}\left[\mathrm{e}^{-\beta H} \mathrm{U}^{\ell}\right]=\sum_{j=1}^{N}\left\langle j+\ell\left|\mathrm{e}^{-\beta H}\right| j\right\rangle=\int_{y(\beta)=y(0)+\frac{2 \pi}{N} \ell} \mathcal{D} y \exp (-S[y]),
$$

where $y(\tau)$ are paths obeying twisted boundary condition. Now, let us undo the twisted boundary condition in favor of a periodic boundary condition, and insert its effects into the action. Define

$$
q(\tau)=y(\tau)-\frac{2 \pi \ell}{N \beta} \tau, \quad \text { hence } q(\beta)=q(0) \bmod 2 \pi .
$$


Plugging this into original action $S[y]$, we find

$$
S[q, \ell]=\frac{1}{g} \int d \tau\left[\frac{1}{2}\left(\dot{q}+\frac{2 \pi \ell}{N \beta}\right)^{2}-\cos \left(N q+\frac{2 \pi \ell}{\beta} \tau\right)\right]+\frac{\mathrm{i} \theta}{2 \pi} \int\left(\mathrm{d} q+\frac{2 \pi \ell}{N \beta} \mathrm{d} \tau\right)
$$

which agrees exactly with (2.23), with the identification:

$$
A^{(1)}=\frac{2 \pi \ell}{N \beta} \mathrm{d} \tau, \quad A^{(0)}=\frac{2 \pi \ell}{\beta} \tau
$$

Happily, these obey the conditions for a background $\mathbb{Z}_{N}$ gauge field (2.18).

So, the twisted boundary conditions in (2.24) are traded for a background field, and the partition function can equivalently be written as:

$$
Z_{\ell}=\int_{q(\beta)=q(0)} \mathcal{D} q \exp (-S[q, \ell]),
$$

Note the explicit time dependence in the action (2.26). This is not a fluke, it is in fact exactly as it should. This is the classical background that we are dealing with.

One final remark concerns instanton equations in the $\mathbb{Z}_{N}$ background field. Using Bogomolny factorization with action (2.23), we find

$$
\left(\mathrm{d} q+A^{(1)}\right)= \pm\left[2 V\left(N q+A^{(0)}\right)\right]^{1 / 2}, \quad q(\beta)=q(0) \bmod 2 \pi .
$$

which is nothing but

$$
\dot{y}= \pm[2 V(N y)]^{1 / 2}, \quad y(\beta)=y(0)+\frac{2 \pi}{N} \bmod 2 \pi .
$$

in the $y$ parametrization. So, to find the fractional instanton solution, we can work with either parametrization. Either request periodic path solutions with background fields or paths solving (2.30) and satisfying twisted boundary conditions. These two simply related interpretation will help us to figure out a non-trivial solution in $\operatorname{PSU}(N)$ bundle in gauge theories on $\mathbb{R}^{3} \times S^{1}$.

In the formulation where $q(\tau)$ is a periodic path in the $\left(A^{(1)}, A^{(0)}\right)$ background $(2.23)$, the topological term is

$$
\left.W=\frac{1}{2 \pi} \int\left(\mathrm{d} q+A^{(1)}\right)\right)=\underbrace{\frac{1}{2 \pi} \int \mathrm{d} q}_{\in \mathbb{Z}}+\underbrace{\frac{1}{2 \pi N} \int \mathrm{d} A^{(0)}}_{\in \frac{1}{N} \mathbb{Z}} \in \frac{1}{N} \mathbb{Z}
$$

where the first term is integer valued as it is over periodic paths, and the winding number is fractional due to background. In the formulation where $y$ is a path satisfying twisted boundary condition (2.24),

$$
W=\frac{1}{2 \pi} \int \mathrm{d} y \in \frac{1}{N} \mathbb{Z}
$$

This is now fractional due to the twisted boundary condition of $y(\tau)$ field. The action corresponding to these fractional winding configurations are also fractional $S=\frac{1}{N} S_{I}$ due to BPS bound. 
Revealing fractional instantons formally. The existence of fractional instantons with fractional winding number is obvious from figure 2. Expressing the partition function $Z\left[\left(A^{(1)}, A^{(0)}\right)\right] \equiv Z_{\ell}(\beta, \theta)$ as an fractional instanton sum.

$$
\begin{aligned}
Z_{\ell}(\beta, \theta) & =\operatorname{tr}\left[\mathrm{e}^{-\beta H} \mathrm{U}^{\ell}\right] \\
& =\sum_{k=0}^{N-1} \mathrm{e}^{\mathrm{i} \frac{2 \pi \ell k}{N}} \mathrm{e}^{\xi \cos \frac{\theta+2 \pi k}{N}} \\
& =\left[N \sum_{W \in \mathbb{Z}} \sum_{\bar{n}=0}^{\infty} \frac{\xi^{2 \bar{n}+\ell+W N}}{(\bar{n}+\ell+W N) ! \bar{n} !} \mathrm{e}^{\mathrm{i} W \theta}\right] \mathrm{e}^{\mathrm{i} \frac{\ell}{N} \theta} \\
& =\left[N \sum_{W \in \mathbb{Z}} I_{N W+\ell}(2 \xi) \mathrm{e}^{\mathrm{i} W \theta}\right] \mathrm{e}^{\mathrm{i} \frac{\ell}{N} \theta}
\end{aligned}
$$

The second line immediately emerges from our knowledge of energy spectrum of the theory (2.9) and the fact that eigenstates of the Hamiltonian are just Bloch states, and $[\mathrm{U}, H]=0$. To obtain the third line, we can expand the exponentials and sum over $k$ explicitly. Not surprisingly, this is equivalent to the dilute gas of fractional instantons in the path integral picture. The fourth line is a nice mathematical identity which tells us that series expansion of all modified Bessel functions are actually instanton sums. The sum is over $\bar{n}$, and configurations that contribute are actually $\bar{n}$ fractional anti-instantons, and $(\bar{n}+\ell+W N)$ fractional instantons. The winding number of such a configuration is $W+\frac{\ell}{N}$, and the sum has an overall $\mathrm{e}^{\mathrm{i} \frac{\ell}{N} \theta}$ dependence that arise from the fractional part of winding number. These fractional instantons contribute to transition amplitudes in quantum mechanics.

Note that by turning on $\left(A^{(1)}, A^{(0)}\right) \equiv \ell$ background, we are not changing the theory or its Hilbert space, which, within Born-Oppenheimer approximation is just $N$ dimensional, that we denote as $\mathcal{H}_{N}$. In simple words, we are summing over just transition amplitudes in quantum mechanics for states which are $\ell$ units apart. The construction merely reveals the topological configurations which play important role in the full theory.

Probably, one of the counter-intuitive sounding aspects of this construction (yet widely familiar from the symmetric double-well potential, see [51]) is following. We keep repeating that single fractional instanton $\mathcal{I}_{j}$ is not contributing to the partition function, as it is not a periodic path. Yet, the energy gap in the theory is sourced by single fractional instanton effect. The reason is clear. Single fractional instanton gives rise to the transition matrix element between nearest neighbor sites, which in turn determine the splittings in the spectrum of the theory. Say, at $\theta=0$, the gap in the spectrum is given by

$$
E_{\text {gap }}=E_{k=1}-E_{k=0}=-2 K \mathrm{e}^{-S_{I} / N}\left(\cos \frac{2 \pi}{N}-1\right),
$$

a single fractional instanton effect, similar to the gap in the symmetric double-well.

\subsection{Uses of gauging $\mathbb{Z}_{N}$}

Discrete theta angle $\theta_{p}=$ level $p$ Chern-Simons $=$ picking Bloch state with momentum $\boldsymbol{p}$. Now, we can gauge $\mathbb{Z}_{N}$ symmetry. Physically, gauging declares that 
instead of $q$ being physically equivalent to $q+2 \pi$, it is identified with $q+\frac{2 \pi}{N}$. With this procedure, the target-space circle size is effectively reduced to $\frac{2 \pi}{N}$. In other words, $N$-site model is reduced to 1 -site, and out of $N$ states in the lowest band, only one state survives. Gauging, unlike turning a background as described above, changes the Hilbert space, by diluting it. It reduces $\mathcal{H}_{N}$ down to one state Hilbert space $\mathcal{H}_{1}$.

Gauging $\mathbb{Z}_{N}$ symmetry amounts to summing over $\ell, \sum_{\ell=0}^{N-1} Z_{\ell}$. However, this is not the only choice in summation. In fact, the sum over $Z_{\ell}(\beta)$ can be done in $N$-different ways, corresponding to $N$ discrete $\theta$ angles, $\theta_{p}=\frac{2 \pi p}{N}$. This $\theta_{p}$ is nothing but the $1 \mathrm{~d}$ Chern-Simons term in the original topological gauge theory. As a result,

$$
\begin{aligned}
Z_{\left(T_{N} / \mathbb{Z}_{N}\right)_{p}} & =\int \mathcal{D} A^{(1)} \mathcal{D} A^{(0)} Z\left[\left(A^{(1)}, A^{(0)}\right), p\right] \delta\left(N A^{(1)}-\mathrm{d} A^{(0)}\right) \\
& \equiv \frac{1}{N} \sum_{\ell=0}^{N-1} \mathrm{e}^{-\mathrm{i} \frac{2 \pi \ell p}{N}} Z_{\ell}
\end{aligned}
$$

As already stated, as a result of gauging, the $N$-site model reduce to 1 -site model, and Hilbert space gets diluted by a factor of $N$. Which state survives gauging is dictated by the choice of $\theta_{p}$, or equivalently, Chern-Simons level $p$. Using (2.33)

$$
\begin{aligned}
Z_{\left(T_{N} / \mathbb{Z}_{N}\right)_{p}} & =\frac{1}{N} \sum_{\ell=0}^{N-1} \mathrm{e}^{-\mathrm{i} \frac{2 \pi \ell p}{N}} Z_{\ell}(\beta) \\
& =\frac{1}{N} \sum_{\ell=0}^{N-1} \mathrm{e}^{-\mathrm{i} \frac{2 \pi \ell p}{N}} \sum_{k=0}^{N-1} \mathrm{e}^{\mathrm{i} \frac{2 \pi \ell k}{N}} \mathrm{e}^{\xi \cos \frac{\theta+2 \pi k}{N}} \\
& =\sum_{k=0}^{N-1} \delta_{p k} \mathrm{e}^{\xi \cos \frac{\theta+2 \pi k}{N}} \\
& =\mathrm{e}^{\xi \cos \frac{\theta+2 \pi p}{N}}
\end{aligned}
$$

Only the Bloch state with momentum $\frac{2 \pi p}{N}$ in the original Brillouin zone of the $N$-site model survives in the Born-Oppenheimer approximation.

If we relax Born-Oppenheimer approximation and consider the whole Hilbert space, then, the gauging procedure will pick just one-state with Bloch momentum $p$ from each band. In this sense, the whole Hilbert space gets diluted by a factor of $N$.

Finally, one can formalize the connection between the topological configurations in the $\left(T_{N} / \mathbb{Z}_{N}\right)_{p}$ model, the $T_{N}$ model with background $\left(A^{(1)}, A^{(0)}\right) \equiv \ell$ or the original theory without background fields. This is one of the crux of the matter that is most important in gauge theory where things are a bit more involved. The object that we would identify as instanton with winding number 1 and action $S_{0}$ in the $\left(T_{N} / \mathbb{Z}_{N}\right)$ model is the fractional instanton with winding number $\frac{1}{N}$ and action $S_{0}=\frac{S_{I}}{N}$ of the $T_{N}$ model. Therefore, $\left(T_{N} / \mathbb{Z}_{N}\right)_{p}$ theory has non-perturbative data that is relevant to the original $T_{N}$ theory.

Let us make this a bit more precise. When we gauge $\mathbb{Z}_{N}$, and sum over all $\left(A^{(1)}, A^{(0)}\right)$, (instead of just turning on a fixed classical $\mathbb{Z}_{N}$ background), the distinction between isolated minima and fractional instantons $\mathcal{I}_{j}$ disappear. We can in fact call

$$
\mathcal{I}_{j} \mapsto \mathcal{I}_{1}, \forall j \quad \text { by gauging } \mathbb{Z}_{N}
$$


Then, we can make an obstruction vs. admission list:

- Instantons of the form $\left[\mathcal{I}_{1}\right]^{k}, k \neq 0 \bmod N$, which are closed paths in $S^{1} / \mathbb{Z}_{N}$ cannot be lifted to $S^{1}$.

- Instanton anti-instanton pairs of the form $\left[\mathcal{I}_{1}\right]^{k}\left[\overline{\mathcal{I}}_{1}\right]^{k}$ are closed paths in $S^{1} / \mathbb{Z}_{N}$, and they can be lifted to $S^{1}$ with $W=0$. These configurations possess fractional quantized action in the original (ungauged) formulation.

- Instantons in multiples of $N\left[\mathcal{I}_{1}\right]^{N q}, q \in \mathbb{Z}$ can also be lifted to $S^{1}$, with $W=q$. These configurations possess integer quantized action.

These facts are by no means surprising, in fact, rather simple. But this leads us to the following intriguing situation.

- Since in order to find the saddles in $T_{N}$ model, we need to directly look at either

- $T_{N}$ with classical $\mathbb{Z}_{N}$ background $\left(A^{(1)}, A^{(0)}\right)$ or equivalently, $\mathbb{Z}_{N}$ twisted boundary conditions

- $\left(T_{N} / \mathbb{Z}_{N}\right)_{p}$ models where $\mathbb{Z}_{N}$ is gauged

we claim that in order to find the relevant saddles of $\mathrm{SU}(N)$ gauge theories, we should first consider either $\mathrm{SU}(N)$ with background gauge field $\left(B^{(2)}, B^{(1)}\right)$ for the $\mathbb{Z}_{N}^{[1]} 1$ form symmetry or $\left(\mathrm{SU}(N) / \mathbb{Z}_{N}\right)_{p}$ theory where $\mathbb{Z}_{N}^{[1]}$ is gauged. ${ }^{9}$ After finding these configurations, we can patch them up to find the ones that can be lifted to $\mathrm{SU}(N)$ theory. These are fractional action (e.g. $2 S_{I} / N$ ) configurations that contribute to the partition function of $\mathrm{SU}(N)$ theory.

\section{$3 \quad \mathbb{C P}^{N-1}$ in $2 \mathrm{~d}$ and $\mathbb{Z}_{N}$ TQFT}

The $\mathbb{C} \mathbb{P}^{N-1}$ model may be expressed as a U(1) gauge theory with an $N$-component elementary field $z_{i}(x)$, obeying the constraint $\sum_{i=1}^{N}\left|z_{i}(x)\right|^{2}=1$. The theory is invariant under the action of a $\mathrm{SU}(N)$ rotation on the $z_{i}(x)$ field. However, $\mathrm{SU}(N)$ does not act faithfully, since $z_{i}(x)$ is not a gauge invariant operator. The global symmetry that acts faithfully on states in Hilbert space is

$$
G=\mathrm{SU}(N) / \mathbb{Z}_{N}=\operatorname{PSU}(N)
$$

In other words, the $\mathbb{Z}_{N}$ center of the $\mathrm{SU}(N)$ is also part of the $\mathrm{U}(1)$ gauge structure, and should not be counted in the global symmetry. All gauge invariant local operators of the $\mathbb{C P}^{N-1}$ model are in $\operatorname{PSU}(N)$ representations.

We would like to turn on an $\mathrm{SU}(N) / \mathbb{Z}_{N}$ background. To do this, we first introduce a background $\mathrm{SU}(N)$ one-form gauge field $A^{(1)}[3,52]$. Further, to gauge the $\mathbb{Z}_{N}$ part,

\footnotetext{
${ }^{9}$ This is one reason we parallel the notation of quantum mechanics with gauge theory, and emphasize the correspondence: $T_{N} \Leftrightarrow \mathrm{SU}(N)$ and $\left(T_{N} / \mathbb{Z}_{N}\right)_{p} \Leftrightarrow\left(\mathrm{SU}(N) / \mathbb{Z}_{N}\right)_{p}$. The latter systems has non-perturbative data that is relevant to the original systems, where it is interpreted as fractional.
} 
we turn on a pair of $\mathrm{U}(1)$ 2-form and 1-form gauge fields $\left(B^{(2)}, B^{(1)}\right)$, and a Lagrange multiplier $F^{(0)}$ :

$$
Z_{\mathrm{top}, p}=\int \mathcal{D} B^{(2)} \mathcal{D} B^{(1)} \mathcal{D} F^{(0)} \mathrm{e}^{\mathrm{i} \int F^{(0)} \wedge\left(N B^{(2)}-\mathrm{d} B^{(1)}\right)+\mathrm{i} p \int B^{(2)}}
$$

Therefore,

$$
N B^{(2)}=\mathrm{d} B^{(1)}, \quad N \int B^{(2)}=\int \mathrm{d} B^{(1)}=2 \pi \mathbb{Z}
$$

and also promote the $\mathrm{SU}(N)$ background gauge field $A^{(1)}$ to a $\mathrm{U}(N)$ background gauge field $\tilde{A}^{(1)}$ :

$$
\tilde{A}^{(1)}=A^{(1)}+\frac{\mathbb{1}}{N} B^{(1)} \quad \text { (locally) }
$$

The covariant derivative in the $\tilde{A}^{(1)}$ background takes the form

$$
D\left(a, \widetilde{A}^{(1)}\right) z \equiv \mathrm{d} z-\mathrm{i} a z+\mathrm{i} \widetilde{A}^{(1)} z
$$

where $a$ is the original dynamical $\mathrm{U}(1)$ gauge field and $\tilde{A}^{(1)}$ is classical. This couples the $\mathbb{C P}^{N-1}$ model to an $\mathrm{SU}(N) / \mathbb{Z}_{N}$ background field, and the theory now has a 1 -form (background) gauge invariance

$$
B^{(2)} \mapsto B^{(2)}+\mathrm{d} \lambda^{(1)}, \quad B^{(1)} \mapsto B^{(1)}+N \lambda^{(1)}, \quad \widetilde{A}^{(1)} \mapsto \widetilde{A}^{(1)}+\lambda^{(1)}, a \mapsto a+\lambda^{(1)} .
$$

which couples the dynamical field with the classical background. The gauge invariant combination of dynamical and classical fields are given by

$$
\left(a \mathbb{1}-\tilde{A}^{(1)}\right), \quad\left(\mathrm{d} a-B^{(2)}\right)
$$

Therefore, the action of the theory minimally coupled to the background field is given by

$$
S_{\mathrm{bgrd}}=\frac{2}{g^{2}} \int\left[D\left(a, \widetilde{A}^{(1)}\right) z^{\dagger} \wedge * D\left(a, \widetilde{A}^{(1)}\right) z\right]-\mathrm{i} \frac{\theta}{2 \pi} \int\left[\mathrm{d} a-B^{(2)}\right]
$$

BPS instantons can be found by using Bogomolnyi factorization:

$$
D\left(a, \widetilde{A}^{(1)}\right) z= \pm \mathrm{i} * D\left(a, \widetilde{A}^{(1)}\right) z
$$

As a result of classical PSU $(N)$ background, the topological charge and action are no longer quantized in integer units, just like our simple QM problem. Rather, we have, as a perfect counterpart of (2.31),

$$
Q=\frac{1}{2 \pi} \int\left(\mathrm{d} a-B^{(2)}\right)=\underbrace{\int \frac{\mathrm{d} a}{2 \pi}}_{\in \mathbb{Z}}+\underbrace{\frac{1}{N} \int \frac{\mathrm{d} B^{(1)}}{2 \pi}}_{\in \frac{1}{N} \mathbb{Z}} \in \frac{1}{N} \mathbb{Z}
$$

The first term is integer valued because $a$ is in $\mathrm{U}(1)$ bundle. The second term arise from proper quantization of $\frac{\mathrm{d} B^{(1)}}{2 \pi}$. Due to BPS nature of these configurations, their action is fractional $S=\frac{S_{I}}{N}=\frac{4 \pi}{g^{2} N}$. 
Despite the fact that these saddles do not contribute to the partition function of the $\mathbb{C P}^{N-1}$ model without background fields, certain pairs of such configurations, such as

$$
\left(\mathcal{I}_{a}, \overline{\mathcal{I}}_{a}\right) \quad \text { for which } Q=\frac{1}{N}-\frac{1}{N}=0, \quad S=\left(\frac{1}{N}+\frac{1}{N}\right) S_{I}=\frac{2}{N} S_{I}
$$

do. There is no obstruction for them to contribute. Similarly,

$$
\prod_{a=1}^{N} \mathcal{I}_{a} \quad \text { for which } Q=N \times \frac{1}{N}=1 \in \mathbb{Z}, \quad S=N \times \frac{1}{N} S_{I}=S_{I}
$$

also contributes.

More technically, $Q=\frac{1}{N}$ configurations live in a $\operatorname{PSU}(N)$ bundle which cannot be uplifted to an $\mathrm{SU}(N)$ bundle. Yet, composite configurations such as $\left(\mathcal{I}_{a}, \overline{\mathcal{I}}_{a}\right)$ pair and $\prod_{a=1}^{N} \mathcal{I}_{a}$ cary integer topological charge and can be lifted to an $\mathrm{SU}(N)$ bundle.

This argument proves the existence of non-BPS fractional action configurations in the vacuum of the $\mathbb{C P}^{N-1}$ model on arbitrarily large- $T^{2}$, whose non-perturbative significance is controlled by

$$
S_{a}=\frac{S_{\mathcal{I}}}{N}=\frac{4 \pi}{g^{2} N}, \quad \mathcal{I}_{a, 2 d} \sim \mathrm{e}^{-\frac{4 \pi}{g^{2}(\mu) N}+i \frac{\theta}{N}}, \quad \Lambda=\mu \mathcal{I}_{a, 2 d}
$$

where $\Lambda$ is the strong scale of the theory.

The main point is following: even when we consider $\mathbb{C P}^{N-1}$ sigma model without any insertion of $B^{(2)}$ flux, we should still consider the field space in the $\mathrm{U}(1) / \mathbb{Z}_{N}$ bundle, where both topological charge and action are fractional. Then, we should sum over configurations that can be lifted to $\mathrm{U}(1)$ bundle. This is a well-defined mathematical prescription. This, inevitably, leads us to the statement that the non-perturbative expansion parameter in the theory is $\mathrm{e}^{-S_{I} / N}=\mathrm{e}^{-\frac{4 \pi}{g^{2}(\mu) N}}$ and it is exponentially more important than BPST instanton:

$$
\mathrm{e}^{-\frac{S_{I}}{N}} \gg \mathrm{e}^{-2 \frac{S_{I}}{N}} \gg \mathrm{e}^{-3 \frac{S_{I}}{N}} \gg \ldots \gg \underbrace{\mathrm{e}^{-S_{I}}}_{\text {instanton }}
$$

Note that $\gg$ indicates here exponential hierarchies. In particular, at large- $N$ limit, $2 \mathrm{~d}$ instantons scale as $\mathrm{e}^{-\frac{4 \pi}{g^{2}(\mu)}} \sim \mathrm{e}^{-O\left(N^{1}\right)}$ and is completely suppressed, while these configurations persist as $\mathrm{e}^{-\frac{4 \pi}{g^{2}(\mu) N}} \sim \mathrm{e}^{-O\left(N^{0}\right)}$.

\section{1 't Hooft flux using $\frac{(\mathrm{SU}(N) \times \mathrm{U}(1))}{\mathbb{Z}_{N}}$}

In $\mathbb{C P}^{N-1}$ model, by just using U(1) gauge structure, it is impossible to impose 't Hooft's twisted boundary conditions. If we turn on an $\mathrm{SU}(N)$ background field, this also allows us to turn on a non-trivial 't Hooft flux corresponding to twisted boundary conditions, associated with

$$
\frac{\mathrm{SU}(N) \times \mathrm{U}(1)}{\mathbb{Z}_{N}}
$$


Let $\Omega_{\mu}, \omega_{\mu}$ denote the transition functions for the non-abelian and abelian parts. $\Omega_{1}\left(x_{2}\right)$ is the transition function between $\left(x_{1}+L_{1}, x_{2}\right) \sim\left(x_{1}, x_{2}\right) . \Omega_{\mu}$ function is independent of $x_{\mu}$, but depends on other coordinate. For $\mathbb{C P}^{N-1}$ fields, we impose

$$
\begin{aligned}
& z\left(x_{1}+L_{1}, x_{2}\right)=\Omega_{1}\left(x_{2}\right) z\left(x_{1}, x_{2}\right) \omega_{1}^{-1}\left(x_{2}\right), \\
& z\left(x_{1}, x_{2}+L_{2}\right)=\Omega_{2}\left(x_{1}\right) z\left(x_{1}, x_{2}\right) \omega_{2}^{-1}\left(x_{1}\right)
\end{aligned}
$$

where $\omega_{1}\left(x_{2}\right) \equiv \mathrm{e}^{\mathrm{i} \alpha_{1}\left(x_{2}\right)}$ is pure phase. We can connect $z\left(x_{1}+L_{1}, x_{2}+L_{2}\right)$ with $z\left(x_{1}, x_{2}\right)$ in two different ways. For consistency at the corners, the transition functions must satisfy

$$
\begin{aligned}
& \Omega_{1}\left(L_{2}\right) \Omega_{2}(0)=\Omega_{2}\left(L_{1}\right) \Omega_{1}(0) \mathrm{e}^{\mathrm{i} \frac{2 \pi \ell}{N}} \\
& \omega_{1}\left(L_{2}\right) \omega_{2}(0)=\omega_{2}\left(L_{1}\right) \omega_{1}(0) \mathrm{e}^{\mathrm{i} \frac{2 \pi \ell}{N}}
\end{aligned}
$$

corresponding to $\ell=0,1, \ldots, N-1$-units of 't Hooft flux. This is the gauge invariant data in (3.16). The gauge covariance of the flux condition tells us that under a gauge transformation, e.g., $z\left(0, x_{2}\right) \rightarrow g\left(0, x_{2}\right) z\left(0, x_{2}\right) h^{-1}\left(0, x_{2}\right)$, the transition matrices transform as $\Omega_{1}\left(x_{2}\right) \rightarrow g\left(L_{1}, x_{2}\right) \Omega_{1}\left(x_{2}\right) g^{-1}\left(0, x_{2}\right)$ etc. So, there is some gauge freedom in the choice of transition matrices.

As an example, we can satisfy relations (3.17) with the choices $\Omega_{1}\left(x_{2}\right)=\mathrm{e}^{\mathrm{i} \frac{2 \pi T \ell}{N} \frac{x_{2}}{L_{2}}}$, $\Omega_{2}=1, \omega_{1}\left(x_{2}\right)=\mathrm{e}^{\mathrm{i} \frac{2 \pi \ell}{N} \frac{x_{2}}{L_{2}}}, \omega_{2}=1$ where $T=\operatorname{diag}(1, \ldots, 1,-(N-1))$. As a result,

$$
\begin{aligned}
& \Omega_{1}\left(L_{2}\right)=\Omega_{1}(0) \mathrm{e}^{\mathrm{i} \frac{2 \pi \ell}{N}} \\
& \omega_{1}\left(L_{2}\right)=\omega_{1}(0) \mathrm{e}^{\mathrm{i} \frac{2 \pi \ell}{N}}
\end{aligned}
$$

In the $\mathrm{U}(1)$ gauge theory, only $\ell=0$ is allowed. If we turn on just $\mathrm{SU}(N)$ background without turning on a background $\mathbb{Z}_{N}$ gauge field, $\ell$ is still zero. (This does not mean it is uninteresting, see section 3.3.) However, turning on a $\operatorname{PSU}(N)$ background field, we can absorb the aperiodicity of the transition function of one part into the other.

\subsection{Fractional instantons in $\mathbb{C P}^{N-1}$ on large $M_{2}$}

Below, we review the standard textbook construction of instantons and demonstrate how it is modified once a $\operatorname{PSU}(N)$ background is turned on.

Instantons in $2 \mathrm{~d}$ are topologically non-trivial configurations with integer winding number. To see this, note that finite action demands $D_{\mu} z=0$ as $|x| \rightarrow \infty$, a covariantly constant configuration.

$$
z_{i}(x)=n_{i} e^{i \alpha(x)}, n_{i} \bar{n}_{i}=1
$$

where $n_{i}$ is a constant vector with unit norm. Therefore, in the perturbative vacuum,

$$
a=\mathrm{d} \alpha
$$

which are pure gauge configurations. Let $\phi$ parametrize $S_{\infty}^{1}$ boundary of $\mathbb{R}^{2}$. Pure gauge configurations are of the form $\alpha(\phi)=W \phi, W \in \mathbb{Z}$. This gives a mapping from the boundary 
of the physical space to the disjoint classes of gauge transformations, which correspond to homotopy classes $\pi_{1}\left(S^{1}\right)=\mathbb{Z}$. This is the instanton number or topological charge

$$
Q=\frac{1}{2 \pi} \int \mathrm{d} a=\frac{1}{2 \pi} \oint a=\frac{1}{2 \pi} \oint \mathrm{d} \alpha \in \mathbb{Z} .
$$

Assume now we turn on a $\operatorname{PSU}(N)$ background field. Locally, the $\mathrm{U}(N)$ gauge field can be written as:

$$
\mathcal{A}^{(1)}=" \tilde{A}^{(1)}+a \mathbb{1}^{\prime \prime} \quad \text { locally }
$$

where the gauge connection $\mathcal{A}^{(1)}$ is in $\mathrm{U}(N) \cong(\mathrm{U}(1) \times \mathrm{SU}(N)) / \mathbb{Z}_{N}$.

Example. The simplest example of a $\mathrm{U}(N)$ field of this form is following. Consider $F_{12}=$ $\frac{2 \pi \ell_{12}}{N L_{1} L_{2}} T$, with $T=\operatorname{diag}(1,1, \ldots, 1,-(N-1))$ is a Cartan generator, and $f_{12}=-\frac{2 \pi \ell_{12}}{N L_{1} L_{2}} \mathbb{1}$. Hence, $F_{\mathcal{A}^{(1)}}=-\frac{2 \pi \ell_{12}}{L_{1} L_{2}} \operatorname{diag}(0,0, \ldots, 0,1)$ is a $\mathrm{U}(N)$ gauge field strength which cannot be decomposed into $\mathrm{SU}(N)$ and $\mathrm{U}(1)$ parts, but can be decomposed to $\operatorname{PSU}(N)$ and $\mathrm{U}(1) / \mathbb{Z}_{N}$ parts. The corresponding gauge fields are (setting $\left.A_{1}=0, a_{1}=0\right), A_{2}\left(x_{1}\right)=\frac{2 \pi \ell_{12}}{N L_{1} L_{2}} T x_{1}, a_{2}\left(x_{1}\right)=-\frac{2 \pi \ell_{12}}{N L_{1} L_{2}} \mathbb{1} x_{1}$ and the transition functions are given by $\Omega_{1}\left(x_{2}\right)=\mathrm{e}^{-\mathrm{i} \frac{2 \pi \ell_{12}}{N L_{2}} T x_{2}}, \omega_{1}\left(x_{2}\right)=\mathrm{e}^{-\mathrm{i} \frac{2 \pi \ell_{1}}{N L_{2}} \mathbb{1} x_{2}}, \Omega_{2}=1, \omega_{2}=1$. Hence, the transition functions obey (3.17), corresponding to the insertion of $\ell_{12}$ units of 't Hooft flux.

Now, the topological term can be written as

$$
\frac{1}{2 \pi N} \int \operatorname{tr}\left[F_{\mathcal{A}^{(1)}}\right]
$$

This reduces to $(3.22)$ if $\tilde{A}^{(1)}$ is in $\operatorname{SU}(N)$. But if $\tilde{A}^{(1)}$ is a connection in a $\operatorname{PSU}(N)$ bundle which cannot be lifted to an $\mathrm{SU}(N)$ bundle, the topological charge in (3.24) is no longer quantized in integer units, rather it can assume fractional values, multiples of $1 / N$. In this case, the dynamical field $a$ is not a simple $\mathrm{U}(1)$ gauge field, but a $\mathrm{U}(1) / \mathbb{Z}_{N}$ field.

Let us make this abstract argument concrete. For $\mathrm{U}(N)$ gauge structure, the covariantly constant configurations as $|x| \rightarrow \infty$ takes the form:

$$
z_{i}(|x| \rightarrow \infty)=e^{i T(\phi)} e^{i \beta(\phi)} n_{i}
$$

instead of (3.20). We choose

$$
T(\phi)+\beta(\phi)=\frac{\phi}{N}\left(\begin{array}{cccc}
-1 & & & \\
& -1 & & \\
& & \ddots & \\
& & & (N-1)
\end{array}\right)+\frac{\phi}{N} \mathbb{1}=\left(\begin{array}{cccc}
0 & & \\
& 0 & \\
& & \ddots & \\
& & & \phi
\end{array}\right)
$$

such that $e^{i T(\phi)} e^{i \beta(\phi)}$ is a proper $\mathrm{U}(N)$ gauge transformation. However, $\mathrm{e}^{\mathrm{i} T(\phi)}$ does not live in an $\mathrm{SU}(n)$ bundle, rather it is in the PSU $(n)$ bundle. Similarly, $e^{i \beta(\phi)}$ can no longer be characterized by a proper $\mathrm{U}(1)$ gauge transformation, instead lives in $\mathrm{U}(1) / \mathbb{Z}_{N}$.

$$
\begin{aligned}
& \mathrm{e}^{\mathrm{i} T(\phi+2 \pi)}=\mathrm{e}^{\mathrm{i} T(\phi)} \mathrm{e}^{-\mathrm{i} \frac{2 \pi}{N}} \\
& \mathrm{e}^{\mathrm{i} \beta(\phi+2 \pi)}=\mathrm{e}^{\mathrm{i} \beta(\phi)} \mathrm{e}^{+\mathrm{i} \frac{2 \pi}{N}}
\end{aligned}
$$


This is an example of $\tilde{A}^{(1)}$ being a connection in $\operatorname{PSU}(n)$ bundle which cannot be lifted to $\mathrm{SU}(n)$ bundle.

As a result, the dynamical gauge connection $a$ can no longer be characterized by a $\mathrm{U}(1)$ gauge field, and $\int d a$ is not a multiple of $2 \pi$. Instead, the dynamical $a$ and background $\widetilde{A}^{(1)}$ are now intertwined in an inseparable way. Denoting $g(\phi)=\mathrm{e}^{i T(\phi)} \mathrm{e}^{i \beta(\phi)}=$ $\operatorname{Diag}\left(1, \ldots, 1, \mathrm{e}^{\mathrm{i} N \beta(\phi)}\right)$, the topological charge (3.24) can now be written as

$$
\frac{1}{N} \frac{1}{2 \pi} \int \operatorname{tr}\left[-i g^{-1} \mathrm{~d} g\right]=\frac{1}{2 \pi} \int \mathrm{d} \beta=\frac{1}{N} \mathbb{Z}
$$

and it is quantized in units of $\frac{1}{N}$, same as (3.10). Note that this is the counterpart of (2.32) in quantum mechanics. It is clear that $Q=\frac{1}{N}$ is in a $\mathrm{U}(1) / \mathbb{Z}_{N}$ bundle, and cannot be lifted to an $\mathrm{U}(1)$ bundle. Yet, composite configurations such as $\left(\mathcal{I}_{a}, \overline{\mathcal{I}}_{a}\right)$ pair and $\prod_{a=1}^{N} \mathcal{I}_{a}$ can be lifted to $\mathrm{U}(1)$ bundle. As in our quantum mechanics example, these composites are the non-trivial fractional action configurations contributing to the partition function of original $\mathbb{C P}^{N-1}$ even in the absence of $\operatorname{PSU}(N)$ background.

\subsection{Flavor twist and 't Hooft flux, and relation to $\mathbb{R} \times S^{1}$}

In a joint work with Dunne [11], we proved that if $\mathbb{C P}^{N-1}$ model is compactified on a cylinder with an $\mathrm{SU}(N)$ flavor twisted boundary condition, and if the $\mathrm{SU}(N)$ background is symmetric under $\mathbb{Z}_{N}$ shifts up to permutations, i.e,

$$
\begin{aligned}
\tilde{z}\left(x_{1}+L_{1}, x_{2}\right) & =\Omega_{F} \tilde{z}\left(x_{1}, x_{2}\right), \\
\Omega_{F} \sim \operatorname{Diag}\left(\mathrm{e}^{\mathrm{i} 2 \pi \mu_{1}} \ldots, \mathrm{e}^{\mathrm{i} 2 \pi \mu_{N}}\right) & =\operatorname{Diag}\left(1, \mathrm{e}^{\mathrm{i} \frac{2 \pi}{N}}, \ldots, \mathrm{e}^{\mathrm{i} \frac{2 \pi(N-1)}{N}}\right)
\end{aligned}
$$

then there exists instantons with fractional topological charge and action,

$$
\left.W=\frac{1}{N}, \quad S=\frac{1}{N} \frac{4 \pi}{g^{2}} \quad \text { (weak coupling, no } B^{(2)} \text { flux }\right)
$$

in the semi-classical domain on $\mathbb{R} \times S_{L}^{1}$. See also [53-57]. Fractional instantons are also described in earlier work on $\mathbb{C P}^{N-1}$ in [58-62].

It is conjectured that this regime is continuously connected to the theory at large $\mathbb{R} \times S_{L}^{1}$ and $\mathbb{R}^{2}$. The rationale behind this is following. The above construction, in the operator formalism translates to

$$
Z_{\Omega_{F}}=\operatorname{tr}\left[\mathrm{e}^{-L_{1} H} \widehat{\Omega}_{F}\right]
$$

where $\widehat{\Omega}_{F}=\prod_{k=1}^{N} \mathrm{e}^{\mathrm{i} \frac{2 \pi k}{N} \widehat{Q}_{k}}=\mathrm{e}^{\mathrm{i} \sum_{k=1}^{N} \frac{2 \pi k}{N} \widehat{Q}_{k}}$ where $\widehat{Q}_{k}$ is the number operator associated with $z_{k}$ quanta. The Hilbert space of $\mathbb{C P}^{N-1}$ model fills in representation of $\operatorname{PSU}(N)$, singlet, adjoint etc. For example, $\mathrm{e}^{-L_{1} H} \widehat{\Omega}_{F}\left|\left(\bar{z}_{i} z^{j}\right)\right\rangle=\mathrm{e}^{-L_{1} M_{\text {adj }}} \mathrm{e}^{\mathrm{i}(i-j) \frac{2 \pi}{N}}\left|\left(\bar{z}_{i} z^{j}\right)\right\rangle$ and the state sum of these states leads to $(-1) \mathrm{e}^{-L_{1} M_{\text {adj }}}$ instead of $\left(N^{2}-1\right) \mathrm{e}^{-L_{1} M_{\text {adj }}}$ that would occur in thermal state sum. There is also a singlet $\lim _{x \rightarrow y} \bar{z}_{i}(x) \mathrm{e}^{\mathrm{i} \int_{x}^{y} a} z^{i}(y)$ which is almost degenerate with the adjoint. (The difference is $O(1 / N)[63]$ ). As a result, there are extremely powerful spectral cancellation in the Hilbert space $\mathcal{H}$ of $\mathbb{C P}^{N-1}$. In particular, quite remarkably,

$$
\lim _{N \rightarrow \infty} Z_{\Omega_{F}}(L)=\mathrm{e}^{-L E_{\text {ground }}}
$$


which means no state apart from the ground state contributes to the twisted partition function. This is the idea of quantum distillation, which tells us that the $Z_{\Omega_{F}}(L)$ must be an analytic function of $L$ [64], similar to cancellation that takes place in index calculations in supersymmetric theories [29]. Note that the cancellation in the index calculation is a result of supersymmetry, in our case, the theory is bosonic and we engineered the spectral cancellation by using its global symmetry. The fact that this is possible is a remarkable aspect of the theory.

Let us now see if we can learn something deeper from this construction concerning strongly coupled QFT, and non-perturbative configurations that contribute to path integrals. Since anomalies are independent of the strength of the coupling, we can try to take advantage of that. The first hint in this direction is that a mixed anomaly between $\operatorname{PSU}(N)$ and $C$ on $\mathbb{R}^{2}$ survives upon compactification on $\mathbb{R} \times S^{1}$ [52] if and only if $\Omega_{F^{-}}$ twisted boundary conditions (3.29) are used, and appropriate $B^{(2)}=A_{1 \mathrm{~d}}^{(1)} \wedge L^{-1} \mathrm{~d} x_{2}$ background is turned on.

Now, in the presence of $B^{(2)}$ on a general $\mathbf{T}^{2}$, we showed that fractional topological charge and fractional action configurations exist. Importantly, this statement is not restricted to only $S_{\text {large }}^{1} \times S_{\text {small }}^{1}$, but also valid at for $S_{\text {large }}^{1} \times S_{\text {large }}^{1}$, where the long distance theory is necessarily strongly coupled.

To connect weak and strong coupling regimes, we reconsider $B^{(2)}$ flux along with $\Omega_{F}$ background. We use

$$
\begin{aligned}
& \tilde{z}\left(x_{1}+L_{1}, x_{2}\right)=\Omega_{1}^{F}\left(x_{2}\right) \tilde{z}\left(x_{1}, x_{2}\right) \omega_{1}^{-1}\left(x_{2}\right), \\
& \tilde{z}\left(x_{1}, x_{2}+L_{2}\right)=\Omega_{2}\left(x_{1}\right) \tilde{z}\left(x_{1}, x_{2}\right) \omega_{2}^{-1}\left(x_{1}\right)
\end{aligned}
$$

where we embedded both $B^{(2)}$ flux and $\Omega_{F}$ background into transition matrix:

$$
\begin{array}{rlrl}
\Omega_{1}^{F}\left(x_{2}\right) & =\mathrm{e}^{\mathrm{i} \frac{2 \pi T \ell}{N} \frac{x_{2}}{L_{2}}} \Omega_{F}, & & \Omega_{2}\left(x_{1}\right)=1, \\
\omega_{1}\left(x_{2}\right) & =\mathrm{e}^{\mathrm{i} \frac{2 \pi \ell}{N} \frac{x_{2}}{L_{2}}}, & \omega_{2}\left(x_{1}\right)=1
\end{array}
$$

This construction, in the operator formalism, amounts to

$$
Z_{\Omega_{F}, \ell}=\operatorname{tr}\left[\mathrm{e}^{-L_{1} H} \widehat{\Omega}_{F} \widehat{\mathrm{U}}^{\ell}\right]
$$

where we separated $\widehat{\Omega}_{F}$, and $\widehat{U}^{\ell}$ for clarity. In the path integral formalism, we can express it either as twisted boundary conditions, or coupling to a background $\mathbb{Z}_{N}$ TQFT:

$$
\begin{aligned}
Z_{\Omega_{F}, \ell}= & \left.\int_{\tilde{z}\left(L_{1}, x_{2}\right)=\Omega_{1}^{F}\left(x_{2}\right) \tilde{z}\left(0, x_{2}\right) \omega_{1}^{-1}\left(x_{2}\right)} \mathcal{D} \tilde{z} \exp -\left(\frac{2}{g^{2}} \int\left[D(a) \tilde{z}^{\dagger} \wedge * D(a)\right) \tilde{z}\right]-\mathrm{i} \frac{\theta}{2 \pi} \int[\mathrm{d} a]\right) \\
= & \int \mathcal{D} F^{(0)} \int_{z(\beta)=z(0)} \mathcal{D} z \mathrm{e}^{\mathrm{i} \int F^{(0)} \wedge\left(N B^{(2)}-\mathrm{d} B^{(1)}\right)} \\
& \times \exp -\left(\frac{2}{g^{2}} \int\left[D\left(a, \widetilde{A}^{(1)}\right) z^{\dagger} \wedge * D\left(a, \widetilde{A}^{(1)}\right) z\right]-\mathrm{i} \frac{\theta}{2 \pi} \int\left[\mathrm{d} a-B^{(2)}\right]\right)
\end{aligned}
$$

where $\ell$ is 't Hooft flux, and we set discrete theta angle to $p=0$. 
The topological charge in $\mathbb{C P}^{N-1}$ model with $\Omega_{F}$ twist is fractionally quantized $W=$ $\frac{1}{N} \mathbb{Z}$ for two, at first sight, seemingly different reasons. These are

$$
\begin{aligned}
Q=\frac{1}{2 \pi} \int_{\mathrm{tbc}} d a & =\left.\frac{\mu_{i+1}-\mu_{i}}{2 \pi}\right|_{\mu_{i}=\frac{2 \pi}{N}(i-1)}=\frac{1}{N} \\
& =\left.\frac{1}{N} \ell\right|_{\ell=1}=\frac{1}{N}
\end{aligned}
$$

The first equality is the property of $\mathbb{Z}_{N}$ symmetric (up to permutation) background. It does not need the insertion of 't Hooft flux, but without that insertion, it seems to be deformable from $\frac{1}{N} \mathbb{Z}$ topological charge. In what sense, is it robust then? The point is, if we wish to preserve a mixed anomaly that exists on $\mathbb{R}^{2}$ between $\operatorname{PSU}(N)$ and $\mathrm{C}$, this particular background is the unique one which achieves that [52]. The boundary condition (3.29) remains invariant under the intertwined combination of a center transformation along with $\mathbb{Z}_{N}$ cyclic permutation of fields, $z_{i} \rightarrow z_{i+1}[65]$.

The second equality is telling us that in the 't Hooft flux background, topological charge is $\frac{1}{N}$ quantized, regardless of what the value of $\Omega_{F}$ is. Both of these statements are independent of coupling, and also persist at strongly coupled regime on arbitrary size $T^{2}$. Next, we exhibit that this is the case in two examples, one is large- $N$ limit of $\mathbb{C} \mathbb{P}^{N-1}$ and the other is supersymmetric version.

Reminder of tunnelings in $\mathbb{C P}^{N-1}$ model. Here, we would like to detail few more information about tunneling events in $\mathbb{C P}^{N-1}$ model with $\Omega_{F}$ twisted background on small $\mathbb{R}^{1} \times S^{1}$ that we will use later. Ref. [11] also has an obvious reinterpretation on $\mathbb{R}^{2}=\mathbb{R}^{+} \times S^{1}$ with $\Omega_{F}$ and $B^{(2)}$ flux background. In these backgrounds, the classical minima of the model are

$$
z(|x| \rightarrow \infty)=\boldsymbol{e}_{a}, \quad \boldsymbol{e}_{a}=(\ldots, 0, \underbrace{1}_{a^{\text {th }}}, 0, \ldots), \quad a=1, \ldots, N
$$

Let $\tau$ denotes coordinate on $S_{L}^{1}$ where we take $L \rightarrow \infty$. Then, the minimal tunneling events correspond to

$$
\Delta z=z(\tau=L)-z(\tau=0)=\boldsymbol{e}_{a}-\boldsymbol{e}_{a+1}=\boldsymbol{\alpha}_{a}, \quad a=1, \ldots, N
$$

where $\boldsymbol{e}_{N+1} \equiv \boldsymbol{e}_{1}$ and $\boldsymbol{\alpha}_{N}=-\sum_{a=1}^{N-1} \boldsymbol{\alpha}_{a}$ is the affine root of $\mathrm{SU}(N)$ Lie algebra. These fractional instanton events $\mathcal{I}_{a}$ have topological charge $W=1 / N$ and action $S=\frac{S_{I}}{N}=\frac{4 \pi}{g^{2} N}$ and are the semi-classical ingredients of our analysis. The fugacity associated with these events are

$$
\mathcal{I}_{a} \sim \mathrm{e}^{-\frac{4 \pi}{g^{2}(\mu) N}+\mathrm{i} \frac{\theta}{N}}=\Lambda \mu^{-1}
$$

These are minimal tunneling events associated with $\boldsymbol{\alpha}_{a}$. More generally, the set of tunneling events obeying BPS-type equation are $N^{2}-N$ types, and associated with charges

$$
\boldsymbol{\alpha}_{a b}=\boldsymbol{e}_{a}-\boldsymbol{e}_{b}
$$


with topological charge and action

$$
W=(a-b) / N, \quad S=|a-b| \frac{S_{I}}{N}
$$

The usual instanton in $2 \mathrm{~d}[8]$ corresponds to $\mathcal{I}=\prod_{a=1}^{N} \mathcal{I}_{a}$ with charge $\boldsymbol{\alpha}_{1}+\ldots+\boldsymbol{\alpha}_{N}=0$ configurations. We can also construct infinite towers of the fractional instantons with charge $\boldsymbol{\alpha}_{a}$ by merging it with $2 \mathrm{~d}$ instantons, $\mathcal{I}_{a}(\mathcal{I})^{q}, q \in \mathbb{Z}$.

As we emphasized, there are also important bion configurations, minimal versions of which are $\left[\mathcal{I}_{a} \overline{\mathcal{I}}_{a+1}\right]$. These have "Coulomb" charges $\boldsymbol{\alpha}_{a}-\boldsymbol{\alpha}_{a+1}$, topological charge $W=0$, and action $S=2 S_{I} / N$. There are also neutral bions $\left[\mathcal{I}_{a} \overline{\mathcal{I}}_{a}\right]_{ \pm}$which have zero "Coulomb" and topological charge, and $S=2 S_{I} / N$ action.

\subsection{Interpolation between weak and strong coupling holonomy potentials}

Small $\boldsymbol{L}$ holonomy potential. In the $\mathbb{C P}^{N-1}$ model, the gauge field $a$ does not have a kinetic term in the UV-Lagrangian, and is not an independent degree of freedom. It is related to the dynamical fields $z\left(x_{1}, x_{2}\right)$ through equations of motions,

$$
a=\mathrm{i} \bar{z} \mathrm{~d} z
$$

In the small $L \Lambda \lesssim 1$ regime, we can write down the potential for the holonomy field $\mathrm{e}^{\mathrm{i} \oint_{S^{1}}{ }^{a}}$, both with and without the insertion of $\Omega_{F}$. They exhibit a striking difference:

$$
\begin{aligned}
V_{\text {thermal }}(a) & =-\frac{2 N}{\pi L^{2}} \sum_{n=1}^{\infty} \frac{1}{n^{2}} \cos (a L n) \sim-\frac{2 N}{\pi L^{2}} \cos (a L) \\
V_{\Omega_{\mathrm{F}}}(a) & =-\frac{1}{\pi L^{2}} \sum_{n=1}^{\infty} \frac{1}{n^{2}}\left(\operatorname{tr}\left(\Omega_{F}^{n}\right) \mathrm{e}^{\mathrm{i} a L n}+\text { c.c. }\right) \\
& =-\frac{2}{\pi L^{2}} \frac{1}{N} \sum_{n=1}^{\infty} \frac{1}{n^{2}} \cos (N a L n) \sim-\frac{2}{\pi L^{2}} \frac{1}{N} \cos (N a L)
\end{aligned}
$$

where $a L$ is periodic by $2 \pi$. In thermal case, there is a unique minimum in the fundamental domain, and a potential barrier as $a \rightarrow a+2 \pi$ corresponding precisely to tunneling events with $W=1$.

In the presence of the $\Omega_{F}$ twist, we obtain a potential $\sim \cos (N L a)$ just like our $T_{N}$ quantum mechanics, which has $N$-minima at $L a=\frac{2 \pi}{N} j, j=0,1, \ldots, N-1$. The interpolation between these configurations correspond to $W=1 / N$ fractional instanton events.

It is important to note that, since $a$ is not an independent degree of freedom, these minima are some configurations of $z$-field, and tunneling is between $z$ field configurations, which translates to $a$ through (3.43). The profiles of these fields are shown in figure 4 borrowed from [11].

Note that the $\Omega_{F}$ twist is an $\mathrm{SU}(N)$ background. We can also turn an $\mathrm{SU}(N) / \mathbb{Z}_{N}$ background by coupling to $B^{(2)}=A_{1 \mathrm{~d}}^{(1)} \wedge L^{-1} \mathrm{~d} x_{2}$. Then $\ell$ units of $B^{(2)}$ flux amounts to considering $\operatorname{tr}\left[\mathrm{e}^{-L_{1} H} \widehat{\Omega}_{F} \mathrm{U}^{\ell}\right]$ which strictly enforces that a transition between $j$ and $j+\ell$ takes place with topological charge $W=\frac{\ell}{N}$ and action $S=\frac{\ell}{N} \frac{4 \pi}{g^{2}}$. 

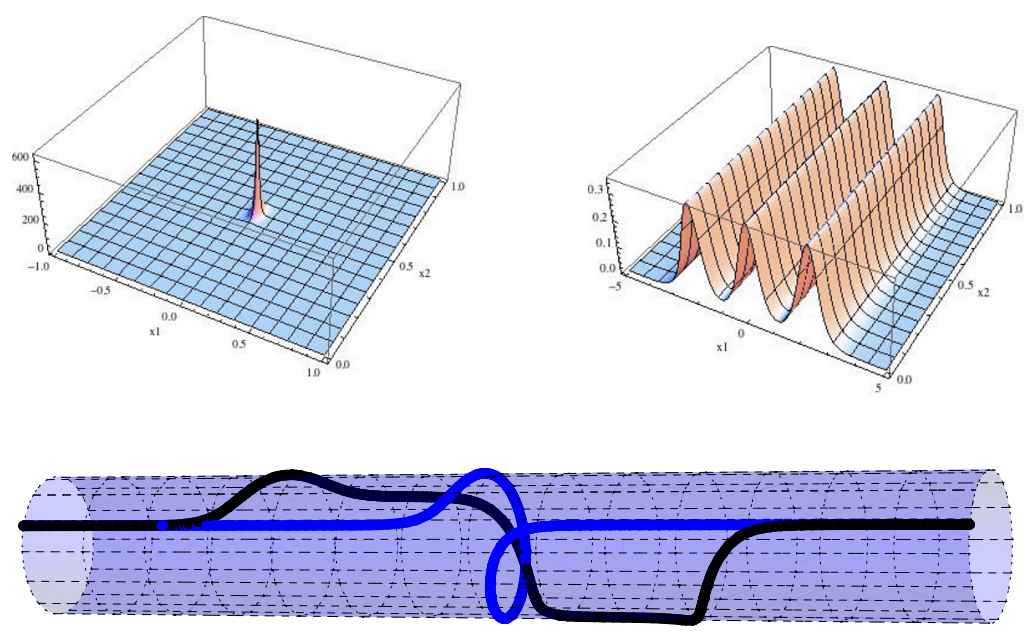

Figure 4. When the moduli parameter of instanton is smaller than the scale set by the $\Omega_{F}$ background, instanton looks like a single lump (first figure), and when the moduli parameter is large, it fractionates to $\mathrm{N}$-pieces (second figure). Corresponding gauge holonomies for small and large instantons are also depicted, demonstrating fractionalization. For these solutions, 't Hooft flux is set to zero. TQFT argument tells us that even when the instanton does not physically split to parts, it can still be viewed as a composite of $N W=1 / N$ fractional instantons. Figure borrowed from [11].

Large $\boldsymbol{L}$ holonomy potential. Now, let us consider large- $L$ limit, where the asymptotically free $\mathbb{C P}^{N-1}$ coupling $g^{2}(\mu)$ necessarily becomes large at distances $|x| \geq \Lambda^{-1}$. A useful handle in this regime is to consider large- $N$ limit. At large- $N$, the theory is solvable [66]. It can be shown to acquire a mass gap dynamically,

$$
M=\Lambda=\mu e^{-4 \pi / N g^{2}(\mu)}
$$

which very much looks like a fractional instanton effect. But the large- $N$ solution is very shy about giving us any insights concerning the microscopic origins of mass.

The saddle of the large- $N$ solution on $\mathbb{R}^{2}$ is $a=0, \lambda=M^{2}$, where $\lambda$ is Lagrange multiplier that appears in the action as $\int \lambda(\bar{z} z-1)$ imposing the constraint $|z|^{2}=1$. A remarkable fact about $\mathbb{C P}^{N-1}$ model is large- $N$ volume independence in the $\Omega_{F}$ twisted background. The large- $N$ saddle point equation on $\mathbb{R}^{2}$ may be derived precisely at any size $\mathbb{R} \times S^{1}$ if and only if one also takes the background $\Omega_{F}[11,67]$.

At large- $N$, we may say that $z$ particles become massive. Ref. [63] showed that these particles generate a kinetic term for the gauge field at one-loop level on $\mathbb{R}^{2}$. The effective Lagrangian is

$$
L_{\mathrm{eff}}=\frac{1}{e^{2}}(\mathrm{~d} a)^{2}+|D z|^{2}+M^{2}|z|^{2}
$$

where $e^{2}=\frac{48 \pi M^{2}}{N}$, and the effective theory is just $N$ - component scalar QED. Consider this theory on $\mathbb{R} \times S_{L}^{1}$. At low temperature $(L \rightarrow \infty)$ for thermal theory, and $\Omega_{F}$-twisted 
(non-thermal) theory, we obtain the holonomy potentials as

$$
\begin{aligned}
V_{\text {thermal }}(a, M) & =-\frac{2 N}{\pi L^{2}} \sum_{n=1}^{\infty} \frac{f_{n}}{n^{2}} \cos (a L n) \approx-\frac{2 N}{L^{2}}\left(\frac{M L}{2 \pi}\right)^{1 / 2} \mathrm{e}^{-M L} \cos (a L) \\
V_{\Omega_{\mathrm{F}}}(a, M) & =-\frac{1}{\pi L^{2}} \sum_{n=1}^{\infty} \frac{f_{n}}{n^{2}}\left(\operatorname{tr}\left(\Omega_{F}^{n}\right) \mathrm{e}^{\mathrm{i} a L n}+\text { c.c. }\right) \\
& =-\frac{2}{\pi L^{2}} \frac{1}{N} \sum_{n=1}^{\infty} \frac{f_{N n}}{n^{2}} \cos (N a L n) \approx-\frac{2}{N L^{2}}\left(\frac{M L N}{2 \pi}\right)^{1 / 2} \mathrm{e}^{-M L N} \cos (N a L)
\end{aligned}
$$

where $f_{n}=(n L M) K_{1}(L M n)$ where $K_{1}$ is the modified Bessel function of the first kind, and in the simplified form, we used asymptotic behavior $K_{1}(z) \sim \sqrt{\frac{\pi}{2 z}} e^{-z}, z \rightarrow \infty$.

The untwisted case is derived by Affleck, who showed the existence of a zero temperature $(L=\infty)$ phase transition, as can be seen by the non-commutativity of $L \rightarrow \infty$, $N \rightarrow \infty$ limits. In the $\Omega_{F}$-twisted case, Sulejmanpasic showed the commutativity of the two limits [67]. In the $\Omega_{F}$ twisted case, we again end up with our $T_{N}$ model, with $N$ inequivalent minima, and instantons again split up to fractional instantons with winding number $W=1 / N$.

The whole discussion in the $L \Lambda \lesssim 1$ domain and $L M N \gg 1$ domains are extremely similar. Note that if we interpret the instanton in $V_{\text {thermal }}(a)$ in (3.47) as quantum instantons in the effective theory according to Affleck with $S \sim N \frac{1}{M L} e^{-M L / 2}$, we should view the instantons in the $V_{\Omega_{\mathrm{F}}}(a)$ as fractional quantum instantons with action $S \sim \frac{1}{M L} \mathrm{e}^{-M L N}$. However, neither detail is actually too important for our purpose. The $a$-field in this description is to a certain extend a place holder, because $e^{2} \sim \frac{M^{2}}{N}$ and electric coupling tend to zero in the large-N limit. Therefore, $a$ is actually a mnemonic of what is going on in the world of $z$-field through the relation (3.43).

The main point is, on a spacetime manifold $T^{2}$, viewed as finite-volume regularization of $\mathbb{R}^{2},(3.31)$ tells us that there are configurations with fractional winding number $\frac{1}{N} \mathbb{Z}$ and action $S=\frac{4 \pi}{g^{2} N}$, for which $a$ lives in the $\mathrm{U}(1) / \mathbb{Z}_{N}$ bundle. To see the existence of these configurations without the insertion of $\left(B^{(2)}, B^{(1)}\right)$ flux, by just using the $\mathrm{SU}(N)$ twist is also possible, and already shown in [11] in semi-classical domain. However, $\left(B^{(2)}, B^{(1)}\right)$ flux tells us that these configurations are there both at weak or strong coupling with certainty. This is just a statment concerning field configuration topology. As we have shown in $T_{N}$ quantum mechanics, we can use these configurations to built fractional action and integer winding configurations which live in the $\mathrm{U}(1)$ bundle. Next, we show that a description based on this idea is actually taking place in 2 d softly broken $\mathcal{N}=(2,2) \mathbb{C P}^{N-1}$ model.

\section{5 $\mathcal{N}=(2,2) \mathbb{C P}^{N-1}$ model on $\mathbb{R}^{2}$ with soft mass}

Consider the $\mathcal{N}=(2,2)$ supersymmetric $\mathbb{C P}^{N-1}$ model. This model has a $\mathbb{Z}_{2 N}$ chiral symmetry which is broken dynamically to $\mathbb{Z}_{2}$ by the formation of fermion-bilinear condensate.

$$
\left\langle k\left|\psi_{-} \psi_{+}\right| k\right\rangle=N \Lambda e^{i \frac{2 \pi k}{N}}, \quad k=0,1, \ldots, N-1
$$

leading to $N$ isolated vacua, consistent with the index $I_{W}=\operatorname{tr}\left((-1)^{F}\right)=N[29]$. 
Consider adding a soft mass term to the Lagrangian, $m \psi_{-} \psi_{+}+$h.c.. In this case, the $k^{\text {th }}$ vacuum energy density is modified into

$$
\mathcal{E}_{k}=-m\left\langle\psi_{-} \psi_{+}\right\rangle_{k}+\text { c.c. }=-2 m N \Lambda \cos \frac{\theta+2 \pi k}{N}
$$

at leading order in $m$. Here $\Lambda=\mu \mathrm{e}^{-S_{I} / N}=\mu \mathrm{e}^{-\frac{4 \pi}{g^{2}(\mu) N}}$ is the renormalization group invariant strong scale of the theory (at one-loop order), and $\mu$ is the (Pauli-Villars) renormalization scale. The graded partition function for the mass deformed theory is

$$
Z(m, \theta)=\operatorname{tr}\left[(-1)^{F} \mathrm{e}^{-L H}\right]
$$

and on a 2-manifold, it is modified as

$$
Z(m, \theta)=\sum_{k=0}^{N-1} \mathrm{e}^{2 m N \Lambda V_{2} \cos \frac{\theta+2 \pi k}{N}}
$$

where $V_{2}$ is the volume of the 2-manifold and $m$ is small. (Assume $V_{2}$ is large compared to strong length scale.) Based on our quantum mechanical example, we can rewrite the partition function as

$$
\begin{aligned}
& Z(m, \theta)=N \sum_{W \in \mathbb{Z}} \sum_{n=0}^{\infty} \sum_{\bar{n}=0}^{\infty} \frac{1}{n !} \frac{1}{\bar{n} !}\left(m N \mu V_{2} \mathrm{e}^{-\frac{S_{I}}{N}+\mathrm{i} \frac{\theta}{N}}\right)^{n}\left(m N \mu V_{2} \mathrm{e}^{-\frac{S_{I}}{N}-\mathrm{i} \frac{\theta}{N}}\right)^{\bar{n}} \delta_{n-\bar{n}-W N, 0} \\
& =N \sum_{W \in \mathbb{Z}}\left[\sum_{\bar{n}=0}^{\infty} \frac{\left(m N \mu V_{2} \mathrm{e}^{-\frac{S_{I}}{N}}\right)^{2 \bar{n}+W N}}{\bar{n} !(\bar{n}+W N) !}\right] \mathrm{e}^{\mathrm{i} W \theta} \\
& =N \sum_{W \in \mathbb{Z}}\left[I_{N W}\left(2 m N \mu V_{2} \mathrm{e}^{-\frac{S_{I}}{N}}\right)\right] \mathrm{e}^{\mathrm{i} W \theta} \quad \text { (strong coupling, no } B^{(2)} \text { flux) }
\end{aligned}
$$

The interpretation of this formula in the light of our discussion of $\mathbb{C P}^{N-1}$ model is following.

- Terms in the sum are sourced by the solution of self-duality equation in the $\mathbb{C P}^{N-1}$ theory with $\operatorname{PSU}(N)$ background. However, these cannot directly contribute to the partition function as they live in a part of $\operatorname{PSU}(N)$ bundle that cannot be lifted to $\mathrm{SU}(N)$ bundle. Happily, the constraint tells us that they do not.

- The constraint $\delta_{n-\bar{n}-W N, 0}$ guarantees that non-BPS configurations in the $\operatorname{PSU}(N)$ bundle that can be uplifted to $\mathrm{SU}(N)$ bundle contribute to the sum. The sum consistently reduces to a sum over integer topological charge configurations $W \in \mathbb{Z}$, but there are fractional action configurations $S=\frac{2}{N} S_{I}$ contributing to it.

- In the massless theory, the chiral symmetry is $\mathbb{Z}_{2 N}$. In the $\operatorname{PSU}(N)$ background, each of these objects carries 2 zero modes. This is not inconsistent with ABJ anomaly thanks to the constraint $\delta_{n-\bar{n}-W N, 0}$. The 2 zero modes is also a natural reflection of mixed anomaly between $\operatorname{PSU}(N)$ and $\mathbb{Z}_{2 N}$. If we were to gauge $\operatorname{PSU}(N)$, we would indeed end up with 2 zero modes with each of these configurations. These zero modes are lifted by the insertion of mass term $m$. 
- The solutions in the $\operatorname{PSU}(N)$ background have 2 bosonic zero modes, and altogether, they should have $2 N$ bosonic zero modes as a $2 d$ BPST instanton. $V_{2}$ may be viewed as the volume of the bosonic moduli space. $\mu$ is Pauli-Villars regularization scale. It appears with the power $\mu^{n_{b}-\frac{n_{f}}{2}}=\mu^{1}$ where $n_{b}=2, n_{f}=2$ are the numbers of bosonic and fermionic moduli.

- The factor $N$ in the argument and order of Bessel function arises because there are $N$ different types of fractional instantons in the $\operatorname{PSU}(N)$ background. Note that at $m=0$, supersymmetric point, modified Bessel obeys $I_{j}(0)=\delta_{j, 0}$ and $Z(m=0, \theta)=$ $N$ which is just supersymmetric index [29]. At $m=0$, the only sector contributing to graded partition function is the zero topological charge sector.

Again, we reach to the same conclusion in $\mathcal{N}=(2,2) \mathbb{C P}^{N-1}$, as in the bosonic model. Even when we consider $\mathbb{C P}^{N-1}$ model without $B^{(2)}$ flux, we should still consider the field space in the $\mathrm{U}(1) / \mathbb{Z}_{N}$ bundle, where both topological charge and action are fractional, and sum up over configurations that can be lifted to $\mathrm{U}(1)$ bundle. This tells us that the non-perturbative expansion parameter in the theory is $\mathrm{e}^{-S_{I} / N}$ which is exponentially more important than $2 \mathrm{~d}$ instantons [8].

Topological susceptibility and how to rescue naive instanton analysis. Despite the fact that (3.52) is a sum over $W \in \mathbb{Z}$ configurations, it leads to topological susceptibility that is in qualitative agreement with the purely bosonic theory

$$
\chi_{\text {top. }}^{\text {soft }}=\left.\frac{\partial^{2} \mathcal{E}}{\partial \theta^{2}}\right|_{\theta=0}=\frac{2 m \Lambda}{N} \quad \text { vs. } \quad \chi_{\text {top. }}^{\text {bos. }}=\frac{3 \Lambda^{2}}{\pi N}
$$

The result in bosonic theory is based on large- $N$ solution (see [68], and [23] for a pedagogical description) and lattice simulations [69]. We expect, once, $m \geq \Lambda, \chi_{\text {top. }}^{\text {soft }}$ to saturate to $\chi_{\text {top. }}^{\text {bos. }}$. The important things is both are of order $1 / N$ as they should, as opposed to $\mathrm{e}^{-O(N)}$ as predicted by naive instanton analysis.

In the standard instanton analysis in $2 \mathrm{~d}$ that we learn from textbooks, one is instructed to sum over all integer topological charge $W \in \mathbb{Z}$ configurations with action $S=|W| S_{I}[20,22]$ :

$$
\begin{aligned}
Z_{\text {ins. gas }}(\theta) & =\sum_{n=0}^{\infty} \sum_{\bar{n}=0}^{\infty} \frac{1}{n !} \frac{1}{\bar{n} !}\left(V_{2} K \mathrm{e}^{-S_{I}+\mathrm{i} \theta}\right)^{n}\left(V_{2} K \mathrm{e}^{-S_{I}-\mathrm{i} \theta}\right)^{\bar{n}} \\
& =\sum_{W \in Z}\left[\sum_{\bar{n}=0}^{\infty} \frac{1}{(\bar{n}+W) !} \frac{1}{\bar{n} !}\left(V_{2} K \mathrm{e}^{-S_{I}}\right)^{2 \bar{n}+W}\right] \mathrm{e}^{\mathrm{i} W \theta} \\
& =\sum_{W \in Z} I_{W}\left(V_{2} K \mathrm{e}^{-S_{I}}\right) \mathrm{e}^{\mathrm{i} W \theta} \\
& =\mathrm{e}^{V_{2} K \mathrm{e}^{-S_{I}} \cos \theta}
\end{aligned}
$$

leading to vacuum energy density and topological susceptibility

$$
\mathcal{E}(\theta) \sim-K \mathrm{e}^{-S_{I}} \cos \theta \sim-K \mathrm{e}^{-O(N)} \cos \theta, \quad \chi_{\text {top. }}^{\text {naive }} \sim \mathrm{e}^{-O(N)}
$$

which are in clear contradiction with the correct result (3.53). 
In principle, in both (3.54) and (3.52), we are summing over only $W \in \mathbb{Z}$ configurations. What went so wrong in the naive instanton analysis? Of course, the answer is clear. In (3.52), we used the configurations with fractional action and topological charge, but imposed a global constraint on topological charge which restricts the sum to $W \in \mathbb{Z}$. This is equivalent to the statement that we used saddles in the $\mathrm{U}(1) / \mathbb{Z}_{N}$ bundle thanks to TQFT coupling and then, lifted the configurations that obey (3.52) constraint to the $\mathrm{U}(1)$ theory. In this way, we obtain the $\theta$ angle dependence and topological susceptibility correctly. This settles the issue raised in [68] concerning the role of fractional instantons in determination of topological susceptibility.

\section{Continuation of $\mathbb{C P}^{N-1}$ semiclassics to strong coupling}

The discussion of this section is the heart of our idea of connecting weakly coupled calculable domain to strongly coupled domain. Hence, it is important to summarize what we did so far for $\mathbb{C P}^{N-1}$, and state what we will do next.

1) We showed the existence of smooth field configurations with fractional $W=1 / N$ topological charge in the presence of background gauge fields for global symmetries. Their existence follows from the usual Bogomolny argument showing they have minimum action proportional to their (fractional) topological charge.

2) The backgrounds in which you see this fractionalization are topological in the sense that they are related to background gauge fields for discrete $\mathbb{Z}_{N}$ symmetries. One can also gauge the discrete symmetry in question by summing over their background fields weighted by a topological phase called discrete theta angle which effectively projects out all but one fractional topological charge sector, and so gives the same information.

3) There are then configurations which are approximately the sum of many widelyseparated fractional instantons (ie, governed by "saddle points at infinity") in the semiclassical domain [5]. Below, we will present the strong coupling realization of this concept.

4) These configurations can then contribute to the path integral with fractional-instanton action even if background and boundary conditions only allow integer topological charge.

5) It is a matter of dynamics whether these fractional-instanton-action configurations do or do not contribute in an important way to the path integral. But analytic continuation from weak coupling semiclassical expansion (familiar from resurgent transseries expansion [11]) suggests that at strong coupling there might be an effective description in terms of fractional-instanton local fields (ie, fields which create local excitations with the quantum numbers of the fractional-instanton bosonic and fermionic zero modes). This is familiar from Polyakov model [66, 70] and deformed Yang-Mills theory on $\mathbb{R}^{3} \times S^{1}[12,14]$ where dual theory is constructed based on monopole-instantons. However, although this 
information is extremely crucial for us, this is not the approach we will pursue. ${ }^{10}$ Hopefully we will do something more powerful.

6) In refs. [16, 17], Fateev, Frolov, Schwarz, and Berg, Lüscher, considered multiinstantons in $\mathbb{C P}^{1}$ on $\mathbb{R}^{2}$, and parametrized the moduli space of $n$-instanton, which is $4 n$ dimensional, as $2 n$ complex coordinates. Gross uses this parametrization earlier in [25] to show that the interaction between the instanton and anti-instanton is a dipole-dipole interaction in $2 d$, where each dipole is as if it is composed of vortex-anti-vortex pair. There is no explicit physical splitting in this parametrization, (let us call it X-parametrization for brevity where $\mathrm{X}$ is short-hand for position moduli in terms of fractional vortex instantons) for example, a single instanton is still a single lump and not two lumps! The magical thing of this parametrization is that the determinant of the fluctuation operator can be calculated exactly, and muti-instanton gas behaves as if it is a Coulomb gas of vortices interacting each other depending on their charges and position moduli. We will give a review of these works, and close its shortcomings. Of course, one of our main motivation is the consistency between fractional quantization of action and topological charge induced by TQFT coupling, fractional quantization that emerges in weak coupling resurgent expansion and the analysis of determinant of the fluctuation operator in X-parametrization admitting an interpretation as if the theory on $\mathbb{R}^{2}$ can be described in terms of these fractional defects. Our goal is to make this correspondence precise.

7) Inspired from the analysis of refs. [16, 17], consistency in weak coupling semi-classical limits and resurgent expansions, matching of the global symmetries and mixed 't Hooft anomalies, and by the consistency (stability) under RG flow of these descriptions, we propose

- massive $N$-flavor Schwinger $=$ Abelian bosonization $=$ mass-deformed $\mathrm{SU}(N)_{1} \mathrm{WZW}$ models as low-energy description of asymptotically free $\mathbb{C P}^{N-1}$ model on $\mathbb{R}^{2}$.

Remarks. The fractional vortex instantons in all cases are classified with the roots of $\mathrm{SU}(N)$ algebra. In semi-classical limits in each case, the elementary tunneling events are given by:

$$
\begin{aligned}
\Delta_{\text {affine }}^{(1)} & =\left\{\boldsymbol{\alpha}_{1}, \ldots, \boldsymbol{\alpha}_{N}\right\} \\
\boldsymbol{\alpha}_{a} & \in \Gamma_{r} \text { of } \mathrm{SU}(N), \quad \mathcal{I}=\prod_{a=1}^{N} \widetilde{V}_{a}
\end{aligned}
$$

where $\boldsymbol{\alpha}_{N}=-\sum_{a=1}^{N-1} \boldsymbol{\alpha}_{a}$ is the affine root. We can in principle write a dilute gas based on these charges, and also include their correlated events such as $\left[\widetilde{V}_{a} \widetilde{V}_{b}\right]$ or $\left[\widetilde{V}_{a} \widetilde{V}_{b}\right]$ etc, but instead we do something more powerful. We write theories for which all orders nonperturbative expansions are the same, according to our understanding of resurgence. We can call the operators $\widetilde{V}_{a}$ associated with tunneling the dual vertex operators.

\footnotetext{
${ }^{10}$ In particular, weak coupling semi-classical expansions is always hierarchical. On the other hand, strong coupling descriptions are egalitarian. We will comment on this, in retrospect, after our construction is over.
} 
The action of the abelian bosonization of Schwinger model will be written in terms of vertex operators (not dual vertex operators), $V_{a}$, associated with the $\boldsymbol{\nu}_{a} \in \Gamma_{w}$ weights of $\mathrm{SU}(N)$ Lie algebra. As asserted, tunnelings in the abelian bosonized description are associated with the $\boldsymbol{\alpha}_{a} \in \Gamma_{r}$. In particular, abelian bosonization, its non-abelian version, and massive Schwinger model are not effective theories describing proliferations of fractional vortex instantons. The fractioal vortex instantons in these theories are same as the one in $\mathbb{C P}^{N-1}$ model in weak coupling limit.

There is a necessary matching of scales between different theories. For example, the non-perturbative expansion parameter in $\mathbb{C P}^{N-1}$ is $\mathrm{e}^{-S_{I} / N}$, which controls the fugacity of vortex instantons with charges $\boldsymbol{\alpha}_{a} \in \Gamma_{r}$ in semi-classical domain. This maps to mass of the fermion in the Schwinger model, $m_{\psi} \sim \mathrm{e}^{-S_{I} / N}$, which in turn becomes mass of the rebosonized field in bosonization construction, or mass deformation of the WZW model.

The above description is self-consistent, because the mass gap cuts off the IR divergence in the fractional-instanton size modulus, so justifies being analytically connected to a dilute fractional-instanton gas (ie, semiclassical) picture. The renormalization group analysis is independent of $\theta$-angle. In particular, it gives a prediction of gaplessness for $\mathbb{C P}^{1}$ at $\theta=\pi$ as we show in detail. As a result, gap, gaplessness and the $\theta$-angle dependence of mass gap that we find in our formalism are reliable qualitative predictions.

\subsection{Fateev, Frolov, Schwarz, and Berg, Lüscher, in retrospect}

In 1979 , refs. $[16,17]$ came up with a very clever construction in the $\mathbb{C P}^{1}$ model, though as they point out themselves, their method has some issues. (See Polyakov's textbook for an assessment [66].) Our construction partially justifies their work on rigorous grounds, and improves it. We feel it is extremely important to understand this discussion in order to perform a similar analysis in gauge theories on $\mathbb{R}^{4}$. So, below, we will explain and improve the ideas of refs. $[16,17,66]$ and bring our own perspective.

Let us outline refs. $[16,17]$ from our perspective. They consider a $q$-instanton configurations in $\mathbb{C P}^{1}$ model. For $q=1$, an instanton on $\mathbb{R}^{2}$ has 4 bosonic zero modes, which are usually parametrized as 2 position moduli for the center of instanton $a^{\mathrm{c}}$, one size moduli $\rho$, and a $\mathrm{U}(1)$ angular moduli $\phi$. The $q$ multi-instanton solution in $\mathbb{C P}^{1}$ model can be written in terms of a holomorphic function

$$
w(\mathbf{z})=\prod_{i=1}^{q} \frac{\mathbf{z}-a_{i}^{1}}{\mathbf{z}-a_{i}^{2}}
$$

where

$$
\left\{a_{1}^{1}, \ldots, a_{q}^{1}\right\}, \quad\left\{a_{1}^{2}, \ldots, a_{q}^{2}\right\}
$$

are just $4 q$ complex moduli. We will refer to this as X-parametrization, for short. It turns out that this is a very useful parametrization for the combination of the following two reasons. reasons that will become clear. 
1) Despite the fact that, what one may think at first sight, the exact solution for instanton $(q=1)$ does not correspond to physical splitting of an instanton into two distinct lumps. If one inspects the topological charge density (integrand in (4.4)) for the $q=1$ solution, it is clear that this is a one-lump configuration:

$$
Q=\frac{1}{\pi} \int d^{2} x \frac{\left|a^{1}-a^{2}\right|^{2}}{\left(\mid \mathrm{z}-\frac{1}{2}\left(\left.\left(a^{1}+a^{2}\right)\right|^{2}+\left|a^{1}-a^{2}\right|^{2}\right)^{2}\right.}=\frac{1}{\pi} \int d^{2} x \frac{\rho^{2}}{\left(\left|\mathrm{z}-a^{\mathrm{c}}\right|^{2}+\rho^{2}\right)^{2}}=1
$$

centered at $a^{\mathrm{c}}$, with size moduli $\rho$, and not two lumps located at $a^{1}$ and $a^{2}$ :

$$
a^{\mathrm{c}}=\frac{1}{2}\left(a^{1}+a^{2}\right), \quad a^{\mathrm{r}}=\rho \mathrm{e}^{\mathrm{i} \phi}=\left(a^{1}-a^{2}\right)
$$

Compare this with the configurations found on $\mathbb{R} \times S^{1}$ in the $\Omega_{F}$ background. There, when the size moduli $\rho$ is smaller than the length scale set by $\Omega_{F}$, there is a single smooth lump. When the size moduli $\rho$ is larger than the scale set by $\Omega_{F}$, the instanton fractionates to $N$ distinct smooth lumps. See figure 4 and ref. [11].

2) Despite the fact that $q=1$ configuration in (4.2) is a single lump, when the quantum fluctuation determinant around multi-instanton configuration is computed exactly at oneloop order, it admits an elegant interpretation in terms of a Coulomb gas in $2 \mathrm{~d}$, as if each instanton is a dipole of two fractional vortex instantons. The partition function (counting only instantons) can be written as $[16,17,66]$ :

$$
\begin{aligned}
Z_{\text {inst }}= & \sum_{q=0}^{\infty} \frac{\left(K \mathrm{e}^{-S_{I} / 2}\right)^{2 q}}{(q !)^{2}} \int \prod_{j=1}^{q} d^{2} a_{j}^{1} d^{2} a_{j}^{2} \\
& \times \exp \left[\sum_{i<j} \log \left|a_{i}^{1}-a_{j}^{1}\right|^{2}+\sum_{i<j} \log \left|a_{i}^{2}-a_{j}^{2}\right|^{2}-\sum_{i, j} \log \left|a_{i}^{1}-a_{j}^{2}\right|^{2}\right]
\end{aligned}
$$

This suggests the following picture: it is as if we are supposed to think of instantons $\mathcal{I}$ as the composites of fractional vortex instantons on $\mathbb{R}^{2}, V^{1}$, and $V^{2}$. In some sense, the formalism presented in refs. [16, 17] can be viewed as "fractionalization without fractionalization". One can formally write $\mathcal{I} \sim V^{1} V^{2}$, where we assign $V^{1}$ Coulomb charge +1 , and $V^{2}$ charge -1 . We will learn that $V^{2}$ cannot be interpreted as anti-vortex, because if we incorporate theta angle, both $V^{1}$ and $V^{2}$ acquires phases $\mathrm{e}^{\mathrm{i} \frac{\theta}{2}} .{ }^{11}$ There are actually $\bar{V}^{1}$ and $\bar{V}^{2}$ in the

\footnotetext{
${ }^{11}$ The sum which takes only instantons into account (without anti-instantons) is actually meaningful here, but not always meaningful. For example, in 3d Polyakov model, it would not be meaningful because one cannot impose global charge neutrality with only charge +1 monopoles. (Coulomb system with only + charges is not stable.) Here, one can guarantee global charge neutrality thanks to the fact that there are two types of fractional vortex instantons with opposite electric charges, and one can have charge neutrality without taking anti-instantons into account. On $\mathbb{R}^{3} \times S^{1}$ deformed Yang-Mills theory (unlike Polyakov model on $\mathbb{R}^{3}$ ), taking only monopole-instantons into account (without anti-monopoles) is also meaningful for the same reason. There are two kinds of monopoles with charges +1 and -1 , and one does not need antimonopoles to have global charge neutrality. In fact, the $\mathrm{SU}(N)$ theory with only monopoles is integrable. It maps to complex affine Toda theory, which happens to have a real spectrum $($ at $\theta=0)$ and can be viewed as a Hirota bilinear form. See [14] on these subtle issues. Once, one includes both monopoles and anti-monopoles, one obtains (5.5) that we discuss in the next section which is no longer integrable.
} 
game, that are not included at this stage, but easy to incorporate (see below.) So, the system behaves as a Coulomb gas with \pm charges, with fugacities $e^{-S_{I} / 2}$ for fractional vortex-instantons. The Coulomb systems in $2 \mathrm{~d}$ have two phases, a gapless molecular phase and gapped plasma phase. $\mathbb{C P}^{1}$ (at $\theta=0$ ) happens to be in the gapped plasma phase. The fugacity of the fractional instantons emerges as the inverse correlation length of the system, $\mathrm{e}^{-S_{I} / 2} \sim \Lambda$, the strong scale of the theory. Now, interestingly, despite the fact that these arise from smooth instanton configurations with X-parametrization, the final form of the action coincide with the one obtained by Gross in [25] obtained by using merons and intuition.

The instanton partition function $Z_{\text {inst }}$ can be reproduced by using a free massless Dirac field $\psi=\left(\begin{array}{l}\psi_{L} \\ \psi_{R}\end{array}\right)$, and bosonization formulas in $2 d$ (see section 32 of [51])

$$
\sigma_{+}(x)=\bar{\psi}_{L} \psi_{R}(x), \quad \sigma_{-}(x)=\bar{\psi}_{R} \psi_{L}(x), \quad \bar{\psi} \psi=\sigma_{+}+\sigma_{-}
$$

and observing that

$$
\begin{aligned}
& \left\langle\sigma_{+}\left(a_{1}^{1}\right) \ldots \sigma_{+}\left(a_{q}^{1}\right) \sigma_{-}\left(a_{1}^{2}\right) \ldots \sigma_{-}\left(a_{p}^{2}\right)\right\rangle \\
& =\prod_{i<j}\left|a_{i}^{1}-a_{j}^{1}\right|^{2}\left|a_{i}^{2}-a_{j}^{2}\right|^{2} \prod_{i, j}\left|a_{i}^{1}-a_{j}^{2}\right|^{-2} \delta_{p q} \equiv \mathrm{e}^{-V_{\text {Coulomb }}} \delta_{p q} \\
& =\delta_{p q} \exp \left[\sum_{i<j} \log \left|a_{i}^{1}-a_{j}^{1}\right|^{2}+\sum_{i<j} \log \left|a_{i}^{2}-a_{j}^{2}\right|^{2}-\sum_{i, j} \log \left|a_{i}^{1}-a_{j}^{2}\right|^{2}\right]
\end{aligned}
$$

where $\langle\cdot\rangle$ is evaluated by using massless Dirac action. The $\delta_{p q}$ is due to conservation of chirality. As a result, the instanton partition function (4.6) can be written as

$$
Z_{\text {inst }}=\int \mathcal{D} \bar{\psi} \mathcal{D} \psi \quad \exp \left[-\int\left(\bar{\psi} \mathrm{i} \gamma_{\mu} \partial_{\mu} \psi+K \mathrm{e}^{-\frac{S_{I}}{2}} \bar{\psi} \psi\right)\right]
$$

Despite the fact that the inclusion of anti-instantons is presented in refs. $[16,17,66]$ as a conceptual difficulty, it is actually a relatively simple matter now, especially after understanding the role of fractional instanton-antiinstantons in resurgence [11], and we describe their inclusion below. There are few issues with the above formula.

- The reliability (or at least self-consistency) of semi-classical expansion.

- The fermionic theory (at least naively) has a global U(1) symmetry that does not match with the $\mathrm{SU}(2) / \mathbb{Z}_{2}$ symmetry of the original $\mathbb{C P}^{1}$ theory. ${ }^{12}$ Effective field theories must respect the global symmetries of microscopic theories. On the other hand, there is actually hope that this point may be fixable, because abelian bosonization (as opposed to non-abelian bosonization) makes some symmetries extremely nonobvious [71]. There is clearly something to be figured out.

- If we incorporate $\theta$, it will not produce $\theta$ angle physics correctly. But this is obvious, since we did not include anti-instantons.

\footnotetext{
${ }^{12}$ Thanks to Aleksey Cherman for discussions about this point.
} 
What FFS-BL did not know and resurgence at help. The coupling a $\Omega_{F}$-twist and $\mathbb{Z}_{N}$ TQFT makes it manifest that the topological defects with fractional charge $W=1 / N$ and action $S_{I} / N$ exist both in the weak coupling semi-classical domain on $\mathbb{R} \times S^{1}$ and on arbitrary $T^{2}$ at strong coupling. Furthermore, unlike the past times [16, 17], we no longer feel uncomfortable about (fractional or not) instanton anti-instanton pairs because resurgence theory tells us what their effects are, and how they are incorporated correctly and cancel ambiguities in perturbation theory [11]. In particular, when we do semi-classics, we are forced (this is not a choice) to consider the complexification of field space. In the old days, it was thought that the instanton-antiinstanton configurations could not be distinguished from perturbative vacuum, and the quasi-zero mode integration between a fractional instanton-antiinstanton would be ill-defined. Now, we know two facts. Fractional instanton antiinstanton configurations are critical points at infinity (see around (2.6) for a reminder), and in order to calculate their contribution to path integral, we need to do path intergal over the Lefschetz thimble, which is meaningful $[5,11]$. Lefschetz thimble integration at second order in semi-classic gives two types of contributions, uncorrelated and correlated pairs $\frac{\beta^{2}}{2 !}\left[\mathcal{I}_{a}\right]\left[\overline{\mathcal{I}}_{a}\right]+\beta\left[\mathcal{I}_{a} \overline{\mathcal{I}}_{a}\right]_{ \pm}$. In other words, thimble integration is a sophisticated version of cluster expansion, which gives the effect of both uncorrelated pairs as well as correlated pairs. The effect of correlated pair is two-fold ambiguous $\operatorname{Im}\left[\mathcal{I}_{a} \overline{\mathcal{I}}_{a}\right]_{ \pm} \sim \pm \mathrm{e}^{-2 S_{I} / N}$. But there is another ambiguity in the theory that comes upon lateral (left/right) Borel resummation of perturbation theory, which we denote as $\mathbb{B}_{ \pm}$. Remarkable fact that comes from resurgent analysis of $\mathbb{C} \mathbb{P}^{N-1}$ is that

$$
\operatorname{ImB} \mathbb{B}_{ \pm}+\operatorname{Im}\left[\mathcal{I}_{a} \overline{\mathcal{I}}_{a}\right]_{ \pm}=0
$$

i.e., the imaginary ambiguities cancel [11]. This ambiguity cancellation is at order $\mathrm{e}^{-2 S_{I} / N} \sim \Lambda^{2}$ and is the semi-classical realization of 't Hooft's famous IR renormalon puzzle [72] (see also [73]) in $\mathbb{C P}^{N-1}$ model. It should be noted in the analysis below, we do not use resurgence, in fact, we do not even use second order correlated pairs in semi-classics except one occasion where it is strictly necessary to determine the IR-dynamics in $\mathbb{C P}^{1}$ at $\theta=\pi$ around (4.52). But the advantage that resurgence provides is clarity it provides in knowing what various non-BPS configurations are, and what the corresponding operators and their physical effects are, and we do not need to need all thanks to renormalization group. Therefore, in the light of these progress in resurgent semi-classical analysis, we feel encouraged to reconsider the FFS-BL analysis $[16,17]$ on $\mathbb{R}^{2}$, and improve it.

\subsection{Incorporating $\theta$ and fractional anti-instantons into FFS-BL}

Based on (4.6), we view the instanton on $\mathbb{R}^{2}$ as a composite of the two fractional (vortex) instantons. But recall that the topological charge density or action density (4.4) are not a two lump configuration. They can be smoothly be split to two-lumps either using $\Omega_{F}$ twist background, or in the background of $B^{(2)} \mathbb{Z}_{2}$-flux TQFT coupling. Both of these backgrounds introduce a scale and if the moduli parameter becomes larger than a certain scale set by the background, we see fractionalization explicitly. The remarkable fact about the $[16,17]$ analysis is that it reveals something deep about the inner structure of $2 \mathrm{~d}$ instanton via a parametrization and exact computation of the fluctuation determinant. 
Due to its extreme importance in the story, we will also incorporate $\theta$ angle dependence from now on. First, note that incorporating $\theta$ angle in $[16,17]$ modifies $(4.9)$ as $\mathrm{e}^{-\frac{S_{I}}{2}} \rightarrow$ $\mathrm{e}^{-\frac{S_{I}}{2}+\mathrm{i} \frac{\theta}{2}}$. Expanding the exponentials, we can perform the path integral by using (4.8) which tells us that when the number of $\sigma_{+}$and $\sigma_{-}$insertions are not equal, the integration vanishes due to excess chirality. As a result, the fractional vortex instanton sum (4.6) is modified into $\sum_{q=0}^{\infty}(\ldots) \mathrm{e}^{\mathrm{i} q \theta}$, where $q=0,1,2, \ldots$ which indicates that the sum is only over non-negative integer winding instantons (without anti-instantons). The inclusion of $\theta$ modifies the action of refs. $[16,17]$ into

$$
S=\bar{\psi} \mathrm{i} \gamma_{\mu} \partial_{\mu} \psi+K \mathrm{e}^{-\frac{S_{I}}{2}} \mathrm{e}^{\mathrm{i} \frac{\theta}{2}} \bar{\psi} \psi
$$

which looks a bit awkward, because $\mathrm{e}^{\mathrm{i} \frac{\theta}{2}}$ appears in front of the Dirac mass term.

If we were to incorporate our sum based on proliferation of $V_{1}, \bar{V}_{1}$, (instead of $V_{1}, V_{2}$ pair), the effective action would be replaced by $\bar{\psi} \mathrm{i} \gamma_{\mu} \partial_{\mu} \psi+\mathrm{e}^{\mathrm{i} \frac{\theta}{2}} \bar{\psi}_{\mathrm{L}} \psi_{\mathrm{R}}+\mathrm{e}^{-\mathrm{i} \frac{\theta}{2}} \bar{\psi}_{\mathrm{R}} \psi_{\mathrm{L}}$. This can only describe topological charge $Q=0$ sector of the theory.

Let us know take into account both instantons and anti-instantons. We view $\mathcal{I} \sim V_{1} V_{2}$ where $V_{a}$ stands for fractional vortex instanton:

$$
\begin{aligned}
& \mathcal{I} \sim V_{1} V_{2}, \quad V_{1} \sim \mathrm{e}^{-\frac{S_{I}}{2}+\mathrm{i} \frac{\theta}{2}} \sigma_{+}, \quad V_{2} \sim \mathrm{e}^{-\frac{S_{I}}{2}+\mathrm{i} \frac{\theta}{2}} \sigma_{-} \\
& \overline{\mathcal{I}} \sim \bar{V}_{1} \bar{V}_{2}, \quad \bar{V}_{1} \sim \mathrm{e}^{-\frac{S_{I}}{2}-\mathrm{i} \frac{\theta}{2}} \sigma_{-}, \quad \bar{V}_{2} \sim \mathrm{e}^{-\frac{S_{I}}{2}-\mathrm{i} \frac{\theta}{2}} \sigma_{+}
\end{aligned}
$$

The topological and Coulomb charges of these configurations are:

$$
\begin{array}{ll}
V_{1}:\left(+\frac{1}{2},+1\right), & V_{2}:\left(+\frac{1}{2},-1\right) \\
\bar{V}_{1}:\left(-\frac{1}{2},-1\right), & \bar{V}_{2}:\left(-\frac{1}{2},+1\right)
\end{array}
$$

Note that Coulomb charges of $V_{1}$ and $\bar{V}_{2}$ are same, but their topological charge differ in sign. They interact with each other according to Coulomb's law in $2 d$. The proliferations of these events on $\mathbb{R}^{2}$ produce a modified mass term: $V_{1}+V_{2}+\bar{V}_{1}+\bar{V}_{2}$. As a result, we obtain the generalization of (4.11),

$$
Z_{\text {inst } / \text { anti-inst }}=\int \mathcal{D} \bar{\psi} \mathcal{D} \psi \quad \exp \left[-\int\left(\bar{\psi} \mathrm{i} \gamma_{\mu} \partial_{\mu} \psi+2 K \mathrm{e}^{-\frac{S_{I}}{2}} \cos \frac{\theta}{2}(\bar{\psi} \psi)\right)\right]
$$

which is now invariant under $\theta \rightarrow-\theta$. This path integration does reproduce the proliferation of both vortices and anti-vortices with the correct $\theta$ angle dependence, and incorporate all $W \in \mathbb{Z}$ sectors. However, it is not only a sum over configurations of the form $\mathcal{I}^{n}, \overline{\mathcal{I}}^{n}$, it also include contribution of configurations such as $V_{1} \bar{V}_{1}$ and $V_{2} \bar{V}_{2}$. 
Let us now investigate the configurations contributing to the full partition function carefully.

$$
\begin{aligned}
Z= & \int \mathcal{D} \bar{\psi} \mathcal{D} \psi \mathrm{e}^{-\int\left(\bar{\psi} \mathrm{i} \gamma_{\mu} \partial_{\mu} \psi+\lambda\left(\mathrm{e}^{\mathrm{i}} \frac{\theta}{2} \bar{\psi}_{L} \psi_{R}+\mathrm{e}^{\mathrm{i} \frac{\theta}{2}} \bar{\psi}_{R} \psi_{L}+\mathrm{e}^{-\mathrm{i} \frac{\theta}{2}} \bar{\psi}_{R} \psi_{L}+\mathrm{e}^{-\mathrm{i} \frac{\theta}{2}} \bar{\psi}_{L} \psi_{R}\right)\right)} \\
= & \int \mathcal{D} \bar{\psi} \mathcal{D} \psi \mathrm{e}^{-\int\left(\bar{\psi} \mathrm{i} \gamma_{\mu} \partial_{\mu} \psi\right)} \\
& \times \sum_{n_{1}=0}^{\infty} \frac{\left(\mathrm{e}^{\mathrm{i} \frac{\theta}{2}} \lambda\right)^{n_{1}}}{n_{1} !} \int\left(\prod_{j=1}^{n_{1}} d^{2} a_{j}^{1}\right) \sigma_{+}\left(a_{1}^{1}\right) \ldots \sigma_{+}\left(a_{n_{1}}^{1}\right) \\
& \times \sum_{n_{2}=0}^{\infty} \frac{\left(\mathrm{e}^{\mathrm{i} \frac{\theta}{2}} \lambda\right)^{n_{2}}}{n_{2} !} \int\left(\prod_{j=1}^{n_{2}} d^{2} a_{j}^{2}\right) \sigma_{-}\left(a_{1}^{2}\right) \ldots \sigma_{-}\left(a_{n_{2}}^{2}\right) \\
& \times \sum_{\bar{n}_{1}=0}^{\infty} \frac{\left(\mathrm{e}^{-\mathrm{i} \frac{\theta}{2}} \lambda\right)^{\bar{n}_{1}}}{\bar{n}_{1} !}\left(\prod_{j=1}^{\bar{n}_{1}} d^{2} b_{j}^{1}\right) \sigma_{-}\left(b_{1}^{1}\right) \ldots \sigma_{-}\left(b_{\bar{n}_{1}}^{1}\right) \\
& \times \sum_{\bar{n}_{2}=0}^{\infty} \frac{\left(\mathrm{e}^{-\mathrm{i} \frac{\theta}{2}} \lambda\right)^{\bar{n}_{2}}}{\bar{n}_{2} !}\left(\prod_{j=1}^{\bar{n}_{2}} d^{2} b_{j}^{2}\right) \sigma_{+}\left(b_{1}^{2}\right) \ldots \sigma_{+}\left(b_{\bar{n}_{2}}^{2}\right)
\end{aligned}
$$

A typical term in the sum has $\left(n_{1}, n_{2}, \bar{n}_{1}, \bar{n}_{2}\right)$ many $\left(V_{1}, V_{2}, \bar{V}_{1}, \bar{V}_{2}\right)$ insertions, respectively. In (4.15), the number of $\sigma_{+}$insertions is $n_{1}+\bar{n}_{2}$ and the number of $\sigma_{-}$insertions is $n_{2}+\bar{n}_{1}$. Therefore, using (4.8), we observe that the sum is non-zero if and only if

$$
n_{1}-\bar{n}_{1}=n_{2}-\bar{n}_{2}=W \in \mathbb{Z}
$$

constraint is obeyed. We can solve this one constraint to turn four sums into triple sum one of which can be identified as a topological charge $W \in \mathbb{Z}$ :

$$
\begin{aligned}
Z= & \sum_{W \in \mathbb{Z}}\left[\sum_{\bar{n}_{1}=0}^{\infty} \sum_{\bar{n}_{2}=0}^{\infty} \frac{\lambda^{2 \bar{n}_{1}+2 \bar{n}_{2}+2 W}}{\left(\bar{n}_{1}+W\right) ! \bar{n}_{1} !\left(\bar{n}_{2}+W\right) ! \bar{n}_{2} !}\right. \\
& \left.\times \int \prod_{j=1}^{n_{1}} d^{2} a_{j}^{1} \prod_{j=1}^{n_{2}} d^{2} a_{j}^{2} \prod_{j=1}^{\bar{n}_{1}} d^{2} b_{j}^{1} \prod_{j=1}^{\bar{n}_{2}} d^{2} b_{j}^{2} \mathrm{e}^{-S_{\text {int }}}\right]_{n_{a}=\bar{n}_{a}+W} \mathrm{e}^{i W \theta}
\end{aligned}
$$

where the interaction action is:

$$
S_{\mathrm{int}}=4 \pi \sum_{i=1}^{2} q_{i} \cdot q_{j}\left[\sum_{k=1}^{n_{i}} \sum_{l=1}^{n_{j}} G\left(a_{k}^{i}-a_{l}^{j}\right)+\sum_{k=1}^{\bar{n}_{i}} \sum_{l=1}^{\bar{n}_{j}} G\left(b_{k}^{i}-b_{l}^{j}\right)-2 \sum_{k=1}^{n_{i}} \sum_{l=1}^{\bar{n}_{j}} G\left(a_{k}^{i}-b_{l}^{j}\right)\right]_{n_{i}=\bar{n}_{i}+W}
$$

where $G(a)=-\frac{1}{4 \pi} \log |a|^{2}$ is $2 \mathrm{~d}$ Green's function.

Now, if we take (4.14) seriously, assuming the semi-classics that leads to it is reliable (we will question this later), it tells us that the $\mathbb{C P}^{1}$ theory on $\mathbb{R}^{2}$ develops a finite correlation length for $\theta \neq 0$, a mass gap, of the form:

$$
m_{\text {gap }}(\theta) \underbrace{=}_{?} \Lambda\left|\cos \frac{\theta}{2}\right|
$$


This is, of course, a beautiful result at first sight, compliant with all of our educated guesses, a non-perturbative mass gap at $\theta \neq \pi$ and gaplessness at $\theta=\pi$, and a wonderful confirmation of the formalism described in refs. [16, 17], and [66]. But this formula (not its consequences) (4.19) is actually not quite correct, because the formalism we set-up to this point, has some flaws.

First, let us describe why we got an "correct looking" result. The partition function has the form $Z=\sum_{W \in \mathbb{Z}} Z_{W} \mathrm{e}^{i W \theta}$ where $Z_{W}$ is the partition function of a fixed $W \in \mathbb{Z}$ sector. But contributing configurations is richer than just naive instanton sums. Clearly, there are configurations of the form $V_{1} \bar{V}_{1}$ with action $\mathrm{e}^{-2 \times \frac{S_{I}}{N}}$ which happens to be just instanton action for $N=2$, but for general $N$, fractional action configurations. At any rate, even for $N=2$, this configuration is distinct from $2 \mathrm{~d}$ instanton [8], which is $V_{1} V_{2}$. The fact that configurations such as $V_{1}$ here, and $\bar{V}_{1}$ there contribute to path integral is responsible for the appearance of $\cos \frac{\theta}{2}$ in the effective action. With the usual instantons, we could never get such a factor. This is a good part of the truth.

Polyakov, in his book [66], following a proposal of ref. [74], argues that to incorporate anti-instantons, one should include a second Dirac fermion and couple the two in a special way. This is by no means necessary. The Coulomb charge of $V_{1}$ is same as the Coulomb charge of $\bar{V}_{2}$. At $\theta=0$, there is no distinction between the corresponding defects and the corresponding operators. Only when we turn on $\theta$, we see their difference due to topology $V_{1} \sim \mathrm{e}^{-\frac{S_{I}}{2}+\mathrm{i} \frac{\theta}{2}} \sigma_{+}, \bar{V}_{2} \sim \mathrm{e}^{-\frac{S_{I}}{2}-\mathrm{i} \frac{\theta}{2}} \sigma_{+}$, but this still does not demand introduction of a second fermion, since the Coulomb charges are same. Hence, to produce the correct sum over vortex instantons and anti-instantons, (4.14) is perfectly sufficient as we showed.

A possible issue with (4.9) (or even (4.14)) is that, at least naively, it does not look consistent with the global symmetry of the original $\mathbb{C P}^{1}$ model, which is $\mathrm{SU}(2) / \mathbb{Z}_{2}$. Rather, it has an obvious $\mathrm{U}(1)$ symmetry. But effective field theories must respect the global symmetries of microscopic theories, and there is no negotiation room there. However, similar to abelian bosonization, sometimes symmetries may be obscured. Still, we want to make global symmetry manifest.

Furthermore, the summation over fractional vortex instantons $V_{a}, \bar{V}_{a}$ is a good approximation if there exists a weak coupling semi-classical domain of the theory, similar to small $S^{1} \times \mathbb{R}$ as in [11]. But here, we are in the strongly coupled domain of the $\mathbb{C} P^{1}$. Is it possible in some way to take into account correlated events such as $\left[V_{a} \bar{V}_{1}\right]_{ \pm},\left[V_{a} \bar{V}_{b}\right],\left[V_{1} V_{1} V_{1}\right], \ldots$ etc. Note that this is not a quantitative, but qualitative issue. For example, does the gaplessness (4.19) persist once higher order effects are taken into account? Or can the theta dependence include terms of the form $\cos \frac{3 \theta}{2}$, or include fractional powers such as $\left(\cos \frac{\theta}{2}\right)^{1 / 3}$ ? If one can achieve this, there is a hope to go beyond semi-classical ideas via such construction.

\subsection{Fermionization, Abelian rebosonization and mass gap at arbitrary $N$}

We are largely inspired from the fermionic description of the fractional vortex instanton gas $[16,17]$ in $\mathbb{C P}^{1}$ model. However, this construction comes with a number of deficits, a number of which we already fixed. However, probably the most important issue is that 
original $\mathbb{C P}^{1}$ has a $\mathrm{SU}(2) / \mathbb{Z}_{2}$ symmetry, which is not manifest in (4.9) (or (4.14)). In more general $\mathbb{C} \mathbb{P}^{N-1}$ case, this symmetry is $\mathrm{SU}(N) / \mathbb{Z}_{N}$, and it is important to capture the mixed anomaly structure of these theories correctly. Therefore, we introduce $N$ massive fermions and gauge over-all U(1) in the general case. Therefore, we land on the $N$-flavor Schwinger model with an $\mathrm{SU}(N)$ invariant mass term.

$$
S=\frac{1}{2 e^{2}} \int_{M_{2}}|\mathrm{~d} a|^{2}+\frac{\mathrm{i} \theta}{2 \pi} \int_{M_{2}} \mathrm{~d} a+\int_{M_{2}} \mathrm{~d}^{2} x \overline{\boldsymbol{\psi}} \gamma^{\mu}\left(\partial_{\mu}+\mathrm{i} a_{\mu}\right) \boldsymbol{\psi}+m \overline{\boldsymbol{\psi}} \boldsymbol{\psi}
$$

The global symmetry, mixed anomalies and global inconsistencies, weak coupling semiclassical description of the $N$-flavor Schwinger model match exactly with the $\mathbb{C} P^{N-1}$ model. Furthermore, 2-flavor Schwinger model provides the correct fix for the result of refs. [16, 17] on $\mathbb{R}^{2}$ based on the $\mathrm{X}$-parametrization of moduli space. Because of these matching, it is natural that the low energy theory for the $\mathbb{C P}^{N-1}$ model is described by massive Schwinger model, or equivalently, its abelian bosonization or its non-abelian bosonization given by mass deformed $\mathrm{SU}(N)_{1} \mathrm{WZW}$ model with an extra scalar. The global symmetry in all cases is $G=\mathrm{SU}(N) / \mathbb{Z}_{N}$ These theories also have charge conjugation symmetry C at $\theta=0, \pi$. The mixed anomaly and global inconsistency are exactly the same in these theories [64, 75].

We will now show that, upon bosonization, the tunneling events in this theory are described by the same set as in $\mathbb{C P}^{N-1}$ model, by (3.39) and (3.41). Later, when we describe semi-classics, we will observe that the mass of the fermion $m$ and the coupling $e^{2}$ in Schwinger model are controlled by strong scale $\Lambda=\mu \mathrm{e}^{-\frac{4 \pi}{g^{2}(\mu) N}}$ of the $\mathbb{C P}^{N-1}$ model. In particular, $m \sim \Lambda$ and $e^{2}=\frac{48 \pi \Lambda^{2}}{N}$.

First, let us review Coleman's celebrated result, by following his footsteps in [71], with some minor remarks sprinkled here and there. For $N$-Fermi fields, $N$-Bose fields are introduced. The bosonization dictionary is:

$$
\begin{aligned}
\bar{\psi}_{a} \gamma^{\mu} \psi_{a} & \leftrightarrow \pi^{-1 / 2} \epsilon_{\mu \nu} \partial_{\nu} \phi_{a} \\
\bar{\psi}_{a} \psi_{a} & \leftrightarrow-c m N_{m} \cos \left(\sqrt{4 \pi} \phi_{a}\right)
\end{aligned}
$$

where $N_{m}$ denotes normal ordering with respect to mass $m$, and $c$ is numerical constant. There are two useful identities proven in [76] and used in [71]. $N_{m}\left[\Pi^{2}+\left(\partial_{1} \phi\right)^{2}\right]=N_{\mu}\left[\Pi^{2}+\right.$ $\left(\partial_{1} \phi\right)^{2}+$ constant] (just an additive shift to ground state energy, which we can be ignorant about in this work), and more importantly

$$
N_{m} \cos (\beta \phi)=\left(\frac{\mu}{m}\right)^{\beta^{2} / 4 \pi} N_{\mu} \cos (\beta \phi)
$$

This relation encodes multiplicative remormalization of the $\cos (\beta \phi)$ operator, to account for the change of the normal ordering scale.

To obtain the bosonized action, we use the dictionary and integrate out the gauge field.

$$
L=\frac{1}{2} \sum_{a=1}^{N}\left(\mathrm{~d} \phi_{a}\right)^{2}-c m^{2} \sum_{a=1}^{N} \cos \left(\sqrt{4 \pi} \phi_{a}\right)+\frac{e^{2}}{2 \pi} \min _{k}\left(\sum_{a=1}^{N} \phi_{a}-\frac{1}{\sqrt{4 \pi}}(\theta+2 \pi k)\right)^{2}
$$

This is same as Coleman's original [71] for $N=2$, except that we took into account the global aspects of gauge field in integrating it out, hence, $\min _{k}$ structure in the potential. As 
Coleman instructs us, the $\mathrm{SU}(N)$ invariance of the theory is obscured in this presentation, but not lost. If we wanted to keep $\mathrm{SU}(N)$ invariance manifest throughout the discussion, we have to use non-abelian bosonization and mass perturbation of the WZW action. Because of its utility, we will return to this perspective as well.

Define the following transformation:

$$
\begin{aligned}
\widetilde{\phi} & =\frac{1}{\sqrt{N}} \sum_{a=1}^{N} \phi_{a} \equiv \frac{1}{\sqrt{N}} \boldsymbol{e}_{0} \cdot \boldsymbol{\phi} \\
\widetilde{\phi}_{a} & =\phi_{a}-\frac{1}{N} \sum_{a=1}^{N} \phi_{a}=\boldsymbol{\nu}_{a} \cdot \boldsymbol{\phi} \quad a=1, \ldots, N \quad \sum_{a=1}^{N} \widetilde{\phi}_{a}=0
\end{aligned}
$$

where now $N$-fields $\widetilde{\phi}_{a}$ obeys a constraint, hence, representing $N-1$ fields, we can write the kinetic term as $\sum_{a=1}^{N}\left(\mathrm{~d} \widetilde{\phi}_{a}\right)^{2}+\frac{1}{2}(\mathrm{~d} \widetilde{\phi})^{2}=\frac{1}{2} \sum_{a=1}^{N}\left(\mathrm{~d} \phi_{a}\right)^{2}$. As a result, the lagrangian can be written as

$$
\begin{aligned}
L= & \frac{1}{2}(\mathrm{~d} \widetilde{\phi})^{2}+\frac{1}{2} \frac{N e^{2}}{\pi} \min _{k}\left(\widetilde{\phi}-\sqrt{\frac{1}{4 \pi N}}(\theta+2 \pi k)\right)^{2}+ \\
& +\frac{1}{2} \sum_{a=1}^{N}\left(\mathrm{~d} \widetilde{\phi}_{a}\right)^{2}-c m^{2} \sum_{a=1}^{N} \cos \left(\sqrt{\frac{4 \pi}{N}} \widetilde{\phi}+\sqrt{4 \pi} \widetilde{\phi}_{a}\right)
\end{aligned}
$$

$\widetilde{\phi}$ particle has a mass square $M^{2}=\frac{N e^{2}}{\pi}=48 \Lambda^{2}$. In Schwinger model, this mode can be made parametrically heavy compared to other modes. However, since we are performing a matching between $\mathbb{C P}^{N-1}$ and Schwinger model to match their semi-classical description in terms of tunneling events, we know that this mode cannot be made parametrically heavier than the lower lying modes. This mode is likely the $\mathrm{SU}(N)$ singlet mode in the spectrum of $\mathbb{C P}^{N-1}, \lim _{x \rightarrow y} \bar{z}_{i}(x) \mathrm{e}^{\mathrm{i} \int_{x}^{y} a} z^{i}(y)$, which differs from the adjoint by an order $O(1 / N)$ splitting [63]. So, this splitting is more pronounced for $N=2,3$, and not so important at large- $N$ where indeed, the adjoint and singlet become degenerate. With this in mind, let us integrate out the singlet field. Using the renormal ordering (or matching) prescription (4.22) of ref. [71], the low-energy theory takes the form:

$$
L=\frac{1}{2} \sum_{a=1}^{N}\left(\mathrm{~d} \widetilde{\phi}_{a}\right)^{2}-c m^{\frac{N+1}{N}} M^{\frac{N-1}{N}} \sum_{a=1}^{N} \cos \left(\frac{\theta+2 \pi k}{N}+\sqrt{4 \pi} \widetilde{\phi}_{a}\right), \quad \widetilde{\phi}_{N}=-\sum_{a=1}^{N-1} \widetilde{\phi}_{a}
$$

Two mass parameters in this action can be further merged to one, by re-using a renormalordering with respect to mass $m^{\prime}$.

$$
\begin{aligned}
L & =\frac{1}{2}(\mathrm{~d} \phi)^{2}-\left(m^{\prime}\right)^{2} \sum_{a=1}^{N} \cos \left(\sqrt{4 \pi} \boldsymbol{\nu}_{a} \cdot \phi\right)+\ldots \\
m^{\prime}(\theta) & =\left(c m^{\frac{2}{N}} M^{\frac{N-1}{N}} \cos \frac{\theta+2 \pi k}{N}\right)^{\frac{N}{N+1}}
\end{aligned}
$$


This result agrees with refs. [71] for $N=2$ and [77-79] for general $N$. Here, we can regard $\boldsymbol{\phi}$ as an $N$-component vector. The mode associated with $\frac{1}{\sqrt{N}} \boldsymbol{e}_{0} \cdot \boldsymbol{\phi}$ decouples from the rest, making (4.27) particularly easy formulation to work with. ${ }^{13}$

Tunneling events in abelian bosonization description. The elementary tunneling events in (4.27) can be described similar to (3.39), and they correspond to

$$
\Delta \phi=\phi(\tau=L)-\phi(\tau=0)=\sqrt{\pi} \boldsymbol{\alpha}_{a}, \quad a=1, \ldots, N
$$

the simple roots and affine root of $\mathrm{SU}(N)$ algebra living in the affine root system.

$$
\Delta_{\text {affine }}^{(1)}=\left\{\boldsymbol{\alpha}_{1}, \ldots, \boldsymbol{\alpha}_{N}\right\}
$$

The crucial point is that the action of these tunneling events is controlled by $m^{\prime}$. Using the matching conditions with $\mathbb{C P}^{N-1}$ for $m$ and $M$, we observe that the tunneling amplitude is controlled by $\mathrm{e}^{-S_{I} / N}$ in the bosonized version of the Schwinger model.

We can also define tunneling events associated with higher roots, and define the orbits as:

$$
\Delta_{\text {affine }}^{(k)}=\left\{\boldsymbol{\alpha}_{a}+\boldsymbol{\alpha}_{a+1}+\ldots \boldsymbol{\alpha}_{a+k-1} \mid a=1, \ldots N\right\}, \quad k=1, \ldots, N-1
$$

corresponding to deomposition of $N^{2}-N$ roots in total to $N-1$ orbits. Note that $\Delta_{\text {affine }}^{(N)}=\boldsymbol{\alpha}_{a}+\boldsymbol{\alpha}_{a+1}+\ldots \boldsymbol{\alpha}_{a+N-1}=0$. The events in the $k^{\text {th }}$ orbit have fugacity $\mathrm{e}^{-k S_{I} / N}$

The proliferation of the vortices describe a classical two-dimensional Coulomb gas of $N^{2}-N$ types of charges associated with charges $\boldsymbol{\alpha}_{a b}=\boldsymbol{\nu}_{a}-\boldsymbol{\nu}_{b}=\boldsymbol{e}_{a}-\boldsymbol{e}_{b}$ interacting with each other via the $2 \mathrm{~d}$ Coulomb's law,

$$
\pm \boldsymbol{\alpha} \cdot \boldsymbol{\beta} \frac{1}{4 \pi} \log \left|\boldsymbol{a}_{\boldsymbol{\alpha}}-\boldsymbol{a}_{\boldsymbol{\beta}}\right|^{2}
$$

In order to define the operators associated with tunneling events, we need to use abelian duality in $2 \mathrm{~d}$. Using $\mathrm{d} \boldsymbol{\sigma}=\star \mathrm{d} \boldsymbol{\phi}$, we can write the tunneling events as:

$$
\widetilde{V}_{\boldsymbol{\alpha}_{a b}}=\mathrm{e}^{-\frac{|a-b| S_{I}}{N}} \mathrm{e}^{\mathrm{i} \sqrt{\pi} \boldsymbol{\alpha}_{a b} \cdot \boldsymbol{\sigma}}
$$

Note that these events are hierarchical in a semi-classical domain, for example, once we introduce the $\Omega_{F}$ background.

Since both semi-classical expansion and the mixed anomalies of the massive Schwinger model are same with the $\mathbb{C P}^{N-1}$, the massive Schwinger model will provide an qualitatively accurate description of the infrared physics of $\mathbb{C P}^{N-1}$.

\footnotetext{
${ }^{13}$ In the process of completing this paper, we became aware of ref. [80], which gives a description of flag manifold sigma models, where fractional vortex instantons associated with $\nu_{a} \in \Gamma_{w}$ gaps out the theory. It is easy to make a connection between that work and ours by using $\mathrm{SU}(N)_{1} \mathrm{WZW}$ formulation (4.40), and using double-trace deformations [14] to connect WZW to flag manifold, generalizing [81].
} 


\subsection{Fractional instanton renormalization group (FIRG)}

Since $\widetilde{\phi}_{a}$ is a constrained field, we need to be careful in reading off the dimension of the $\cos (\ldots)$ operator. For example, setting $N=2$ on (4.26), case examined in [71], $\widetilde{\phi}_{2}=$ $-\widetilde{\phi}_{1}$, where $\widetilde{\phi}_{1}$ is unconstrained. Then, properly normalizing the kinetic term by a field redefinition,

$$
L=\frac{1}{2}\left(\mathrm{~d} \widetilde{\phi}_{1}\right)^{2}-2\left(m^{\prime}\right)^{2} \cos \left(\sqrt{2 \pi} \widetilde{\phi}_{1}\right)
$$

and dimension of the $\cos (\ldots)$ operator is $\Delta\left[\cos \left(\sqrt{2 \pi} \widetilde{\phi}_{1}\right)\right]=\frac{\beta^{2}}{4 \pi}=\frac{1}{2}$, in agreement with [71]. For general $N$, it is slightly tricky to read off the dimension of $\cos (\ldots)$ operator and for this, the way we parametrized things in (4.27) is useful. In this formula, $\phi$ field is unconstrained, but there is an extra redundant mode in it that decouples from the dynamics. As a result, the dimension of vertex operators are easy to read:

$$
\operatorname{dim}\left(V_{a}\right)=\Delta\left[\mathrm{e}^{\mathrm{i} \sqrt{4 \pi} \boldsymbol{\nu}_{a} \cdot \boldsymbol{\phi}}\right]=\frac{\beta^{2} \boldsymbol{\nu}_{a}^{2}}{4 \pi}=\frac{N-1}{N}
$$

This is consistent with non-abelian bosonization in which the identification is:

$$
\bar{\psi}_{\mathrm{L}, a} \psi_{\mathrm{R}, b} \sim U_{a b}=\mathrm{e}^{\mathrm{i} \sqrt{\frac{4 \pi}{N}} \widetilde{\phi}} g_{a b}
$$

and the dimensions of the corresponding operators are $\Delta\left[\mathrm{e}^{\mathrm{i} \sqrt{\frac{4 \pi}{N}} \widetilde{\phi}}\right]=\frac{1}{N}$ and $\Delta\left[g_{a b}\right]=\frac{N-1}{N}$ where $g$ is $\mathrm{SU}(N)$ valued.

Note that IR scaling in the vertex operator in the large- $N$ limit goes to 1 , same as free field or UV of a fermion bilinear in $2 \mathrm{~d}$. The most dramatic change in the scaling dimension happens for $N=2$, in that case, the scaling of fermion bilinear drops from 1 in the UV to $\frac{1}{2}$ in the IR. This has an interesting effect that we discuss separately for $N=2$ case with $\theta$ angle.

The mass matrix for the $\boldsymbol{\phi}$ field can be found by diagonalizing $\sum_{a}\left(\boldsymbol{\nu}_{a} \cdot \boldsymbol{\phi}\right)^{2}$ matrix. The mass square matrix components are $M_{i j}^{2}=\boldsymbol{\nu}_{a}^{i} \boldsymbol{\nu}_{a}^{j}=\delta_{i j}-\frac{1}{N}$

$$
M^{2}=\mathbf{1}_{N}-\frac{1}{N} \boldsymbol{J}_{N}
$$

where $\boldsymbol{J}_{N}$ is all-one matrix and $\mathbf{1}_{N}$ is identity matrix. $M^{2}$ has $N-1$ eigenvalues which are equal to 1 , for which the eigenvectors are $\boldsymbol{\alpha}_{a}, a=1, \ldots N-1$ and 1 eigenvalue equal to 0 with eigenvector $\boldsymbol{e}_{0}$.

Relevants and irrelevants in infrared dynamics (IR scaling dimensions). Let us list the scaling dimension of various vertex operators. ${ }^{14}$ This will help us to identify which of these operators are relevant in the infrared. It will also help us "tower" of interesting

\footnotetext{
${ }^{14}$ The vertex operators in the effective action (4.42) can be viewed as tunneling events of the effective action based on proliferation of the vortices with charges (4.28) in the $\boldsymbol{\alpha} \in \Gamma_{r}$, based on the dual field $\boldsymbol{\sigma}$ in (4.32).
} 
operators which cary same Coulomb charge (scaling dimension), but higher topological charge.

$$
\begin{aligned}
\operatorname{dim}(1) & =0 \\
\operatorname{dim}\left(V_{a}\right) & =\Delta\left[\mathrm{e}^{\mathrm{i} \sqrt{4 \pi} \boldsymbol{\nu}_{a} \cdot \boldsymbol{\phi}}\right]=\left(\boldsymbol{\nu}_{a}\right)^{2}=\left(1-\frac{1}{N}\right) \\
\operatorname{dim}\left(V_{a} V_{b}\right) & =\Delta\left[\mathrm{e}^{\mathrm{i} \sqrt{4 \pi}\left(\boldsymbol{\nu}_{a}+\boldsymbol{\nu}_{b}\right) \cdot \boldsymbol{\phi}}\right]=\left(\boldsymbol{\nu}_{a}+\boldsymbol{\nu}_{b}\right)^{2}= \\
2\left(1-\frac{2}{N}\right) & a \neq b \\
4\left(1-\frac{1}{N}\right) & a=b \\
\operatorname{dim}\left(V_{a} \bar{V}_{b}\right) & =\Delta\left[\mathrm{e}^{\mathrm{i} \sqrt{4 \pi}\left(\boldsymbol{\nu}_{a}-\boldsymbol{\nu}_{b}\right) \cdot \phi}\right]=\left(\boldsymbol{\nu}_{a}-\boldsymbol{\nu}_{b}\right)^{2}=2 \\
\operatorname{dim}\left(V_{a} V_{b} V_{c}\right) & =\Delta\left[\mathrm{e}^{\mathrm{i} \sqrt{4 \pi}\left(\boldsymbol{\nu}_{a}+\boldsymbol{\nu}_{b}+\boldsymbol{\nu}_{c}\right) \cdot \phi}\right]=\left(\boldsymbol{\nu}_{a}+\boldsymbol{\nu}_{b}+\boldsymbol{\nu}_{c}\right)^{2} \geq 3\left(1-\frac{3}{N}\right) \\
\operatorname{dim}\left(V_{a_{1}} \ldots V_{a_{k}}\right) & =\Delta\left[\mathrm{e}^{\mathrm{i} \sqrt{4 \pi}\left(\boldsymbol{\nu}_{a_{1}}+\ldots+\boldsymbol{\nu}_{a_{k}}\right) \cdot \boldsymbol{\phi}}\right]=\left(\boldsymbol{\nu}_{a_{1}}+\ldots+\boldsymbol{\nu}_{a_{k}}\right)^{2} \geq k\left(1-\frac{k}{N}\right) \\
\operatorname{dim}\left(\prod_{a=1}^{N} V_{a}\right) & =\Delta\left[\mathrm{e}^{\mathrm{i} \sqrt{4 \pi}\left(\boldsymbol{\nu}_{a_{1}}+\ldots+\boldsymbol{\nu}_{a_{N}}\right) \cdot \boldsymbol{\phi}}\right]=0 \\
\left.\operatorname{dim}\left(\prod_{a=1}^{N} V_{a}\right)^{q} V_{b}\right) & =\Delta\left[\mathrm{e}^{\mathrm{i} \sqrt{4 \pi} \boldsymbol{\nu}_{b} \cdot \boldsymbol{\phi}}\right]=\left(1-\frac{1}{N}\right) \\
\operatorname{dim}\left(V_{a_{1}} \ldots V_{a_{k}}\right) & =\operatorname{dim}\left(V_{a_{k+1}} \ldots V_{a_{N}}\right)
\end{aligned}
$$

To be relevant in the renormalization group sense [19], an operator must have $\Delta<2$. These are vertex operators $V_{a}$ with $(\Delta \sim 1)$ and two-vertex operators $\left[V_{a} V_{b}\right]$ and $\left[V_{a} \bar{V}_{b}\right]$ for which $(\Delta \sim 2)$. We can ignore others $(\Delta \gtrsim 3)$ for IR physics, but still need to be careful because of some subtleties, such as $\operatorname{dim}\left(V_{a_{1}}\right)=\operatorname{dim}\left(V_{a_{1}} \ldots V_{a_{N-1}}\right) \equiv \operatorname{dim}\left(V_{a_{N}}\right)$.

Finally, in the large- $N$ limit, only level $k=1$ (and $k=N-1$ ) operators are relevant. $k=2$ and $k=N-2$ are marginal, and the rest is irrelevant. This will allow us to determine the IR physics of the theory sufficiently robustly.

\subsection{Non-abelian bosonization: mass deformed $\mathrm{SU}(N)_{1}$ WZW}

The abelian bosonization reveals crucial amount of information about dynamics, but makes the non-abelian global symmetry of the theory non-obvious. On the other hand, if we use non-Abelian bosonization, the matching of the global symmetries become manifest. Let us describe this briefly.

Non-abelian bosonization maps $N$-flavors of free massless Dirac fermions to $\mathrm{U}(N)_{1}$ WZW model [82-84]. The correspondence between operators is

$$
\psi_{\mathrm{L}} \bar{\psi}_{\mathrm{R}} \sim U
$$

where $U$ is the $\mathrm{U}(N)$-group valued scalar field. The 0-form symmetry of the massless

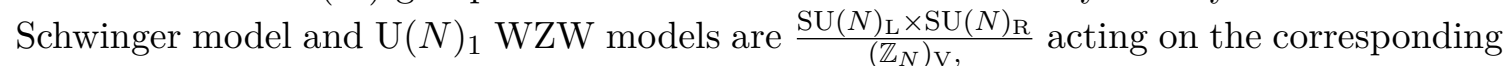
operators by conjugation $(\cdot) \mapsto V_{\mathrm{L}}(\cdot) V_{\mathrm{R}}^{\dagger}$. Turning on a mass term for fermion reduce the 
symmetry to the vectorlike subgroup,

$$
\operatorname{PSU}(N)=\mathrm{SU}(N)_{\mathrm{V}} /\left(\mathbb{Z}_{N}\right)_{\mathrm{V}}
$$

which is the global symmetry of three theories: $\mathbb{C P}^{N-1}$ model, massive $N$-flavor Schwinger model, and mass deformation of SU $(N)_{1}$ WZW model.

The bosonized action of the massive Schwinger model is given by

$$
\begin{aligned}
S= & \frac{1}{2 e^{2}} \int_{M_{2}}|\mathrm{~d} a|^{2}+\frac{1}{8 \pi} \int_{M_{2}} \operatorname{tr}\left(|\mathrm{d} U|^{2}\right)-\frac{c}{2} m^{2}\left(\operatorname{tr} U+\operatorname{tr} U^{\dagger}\right) \\
& +\frac{\mathrm{i}}{12 \pi} \int_{M_{3}} \operatorname{tr}\left(\left(U^{\dagger} \mathrm{d} U\right)^{3}\right)+\frac{1}{2 \pi} \int_{M_{2}} \mathrm{~d} a \wedge(\ln \operatorname{det}(U)+\mathrm{i} \theta),
\end{aligned}
$$

The first two terms are kinetic terms, and the third term is mass deformation, the fourth term is the level-1 Wess-Zumino term. The last term can enforces the ABJ anomaly $\mathrm{U}(1)_{\mathrm{R}} \rightarrow\left(\mathbb{Z}_{N}\right)_{\mathrm{R}}$ of the massless limit, and incorporates theta dependence away from massless point.

Integrating out the gauge field as in the abelian bosonization discussion, we obtain

$$
\begin{aligned}
S= & \frac{1}{8 \pi} \int_{M_{2}} \operatorname{tr}\left(|\mathrm{d} U|^{2}\right)-\frac{c}{2} m^{2}\left(\operatorname{tr} U+\operatorname{tr} U^{\dagger}\right) \\
& +\frac{\mathrm{i}}{12 \pi} \int_{M_{3}} \operatorname{tr}\left(\left(U^{\dagger} \mathrm{d} U\right)^{3}\right)+\frac{e^{2}}{2 \pi} \int_{M_{2}}(\ln \operatorname{det}(U)+\mathrm{i} \theta)^{2},
\end{aligned}
$$

This is the non-abelian version of (4.23) and now, the full PSU( $N)$ symmetry is manifest. We can further (locally) integrate out mode associated with $\operatorname{det}(U)$ to obtain counterpart of (4.26).

According to (4.37), there are very few relevant or marginally relevant deformations of level-1 $\mathrm{SU}(N)$ WZW. These are $\operatorname{tr} U$ with $\Delta \sim 1$ and $\operatorname{tr}\left(U^{2}\right)$ with $\Delta \sim 2$ in large- $N$ limit, and, $|\operatorname{tr} U|^{2},(\operatorname{tr} U)^{2}$ for which $\Delta \sim 2$. In particular, double-trace deformations of the form $\left|\operatorname{tr} U^{n}\right|^{2},\left(\operatorname{tr} U^{n}\right)^{2}$ for which $\Delta \sim 2 n^{2}$, are irrelevant in general for $n \geq 2$.

\subsection{Secrets of Coleman's formula: relation of RG with all orders semi-classics}

In our construction, (4.27) arises as low energy description of the $\mathbb{C P}^{N-1}$ model. For that purpose, we can identify parameters of fermionized low energy limit of $\mathbb{C P}^{N-1}$ as $m \sim \Lambda \sim \mathrm{e}^{-S_{I} / N}$ and $\mu^{2} \sim N e^{2} \sim \Lambda^{2}$ and hence, mass gap is given by

$$
m^{\prime}(\theta)=\Lambda \max _{k}\left(\cos \frac{\theta+2 \pi k}{N}\right)^{\frac{N}{N+1}}
$$

We would like to discuss rich physics associated with this result, which will teach us new things concerning extension of semi-classical ideas to $\mathbb{R}^{2}$. We believe all the major lessons extend to gauge theory on $\mathbb{R}^{4}$ and $\mathbb{R}^{3} \times S^{1}$ and has non-trivial implications.

- Conceptually, the most interesting aspect of Coleman's result [71] is following: naively speaking, if we obtain (4.26) from semi-classical gas of vortices in $S[\boldsymbol{\sigma}]$, we are performing a first order in semi-classic analysis. The Lagrangian has a 
$\cos \left(\frac{\theta}{N}\right) \cos \left(\sqrt{4 \pi} \nu_{a} \cdot \phi\right)$ factor in it which happily arise from the leading order fractional instantons, $V_{a}$. However, in (4.27), we obtain mass gap $\left(\cos \frac{\theta+2 \pi k}{N}\right)^{\frac{N}{N+1}}$ which is impossible to obtain at any finite order in semi-classical expansion. It requires infinite order in semi-classics. What happened in between?

- What Coleman did [71], as he also states, is renormalization group for topological defects $[18,19]$. But renormalization group is smart, even if we just write down few operators that are permitted by symmetries, it will induce all the other operators that are permitted by symmetries. In (4.42), it is actually inducing infinitely many relevant operators and resumming over them!

- What are these infinitely many relevant operators and why did we forget about them at our starting point? The operator $\left(\cos \frac{\theta+2 \pi k}{N}\right)^{\frac{N}{N+1}} \cos \left(\sqrt{4 \pi} \boldsymbol{\nu}_{a} \cdot \boldsymbol{\phi}\right)$ is actually telling us that, apart from the $\mathrm{e}^{\mathrm{i} \sqrt{4 \pi} \nu_{a} \cdot \phi+i \frac{\theta}{N}}$, there are infinitely many other operators of the form $\mathrm{e}^{\mathrm{i} \sqrt{4 \pi} \nu_{a} \cdot \phi+i \theta\left(q+\frac{1}{N}\right)}$, the scaling dimension (or Coulomb charges) of which are the same, but topological charges are distinct, associated with topological defects with winding number $W=\frac{1}{N}(1+N q), q=0,1,2, \ldots$ in the semi-classical language. ${ }^{15}$

Let us consider the $N=2$ case, where the effects is most pronounced. The mass gap as a function of $\theta$ angle in this case is given by: ${ }^{16}$

$$
m_{\text {gap }}(\theta)=\Lambda\left|\cos \frac{\theta}{2}\right|^{\frac{2}{3}}
$$

Let us express $m_{\text {gap }}(\theta)$ as a Fourier series:

$$
m_{\text {gap }}(\theta) \Lambda^{-1}=\sum_{q=0}^{\infty} a_{q} \cos \left(\left(q+\frac{1}{2}\right) \theta\right)
$$

where the Fourier coefficients are given by:

$$
a_{q}=\frac{1}{\pi} \int_{-\pi}^{\pi} \mathrm{d} \theta m_{\text {gap }}(\theta) \Lambda^{-1} \cos \left(\left(q+\frac{1}{2}\right) \theta\right)=-\frac{2^{\frac{4}{3}} \pi}{\sqrt{3} \Gamma\left(-\frac{2}{3}\right) \Gamma\left(\frac{5}{6}-q\right) \Gamma\left(q+\frac{11}{6}\right)}
$$

Numerically, we find

$$
\left\{a_{1}, a_{2}, a_{3}, a_{4}, a_{5}, \ldots\right\}=\{1.07119,-0.09738,0.04009,-0.02266,0.01484, \ldots\}
$$

\footnotetext{
${ }^{15}$ For gauge theory on $\mathbb{R}^{3} \times S^{1}$, each monopole instanton has an infinite KK tower associated with it. If we wish to go beyond semi-classics, the above discussion instructs us that we should some over the whole tower.

${ }^{16}$ In the vicinity of $\theta=\pi$, our construction implies that the mass gap in the $\mathbb{C P}^{1}$ model vanishes in a particular way:

$$
m_{\text {gap }}(\theta)=\Lambda\left|\frac{\theta-\pi}{2}\right|^{\frac{2}{3}}, \quad \theta \rightarrow \pi
$$

If one can overcome the sign problem due to $\theta$ angle, it would be nice to check this prediction of our formalism.
} 
which suggest that even in the case where the leading order semi-classics is supposed to be at its worst $(N=2)$, the numerical error it makes is quite small. In large- $N$, the leading order semi-classics capture the whole result (4.42).

Our intermediate level Lagrangian (4.42) is based on leading vertex operators:

$$
V_{1}, V_{2}, \bar{V}_{1}, \bar{V}_{2}
$$

But renormalization group generates all other (relevant and irrelevant) operators permitted by symmetries. Therefore, we ended up having a theory which describes proliferation of

$$
V_{1}, V_{2}, \bar{V}_{1}, \bar{V}_{2},\left[\left(V_{1} V_{2}\right) V_{1}\right],\left[\left(V_{1} V_{2}\right) V_{2}\right],\left[\left(V_{1} V_{2}\right) \bar{V}_{1}\right],\left[\left(V_{1} V_{2}\right) \bar{V}_{2}\right], \ldots
$$

topological defects with Coulomb charge \pm 1 (which determines the relevance), but arbitrary half integer topological charge, $W \in \frac{1}{2}+\mathbb{Z}$, which tells us that these are sourced by topologically distinct defects:

$$
\left[\left(V_{1} V_{2}\right)^{q} V_{a}\right] \mapsto \mathrm{e}^{\mathrm{i} \sqrt{4 \pi} \widetilde{\phi}_{a}} \mathrm{e}^{\mathrm{i} \frac{1}{2}\left(q+\frac{1}{2}\right) \theta}
$$

There are infinitely many correlated defects, which are relevant in the RG sense. It is natural that these defects generate the non-analytic structure that we see in Coleman's theta angle dependence (4.27). These contribution arise from the different columns in the resurgence triangle, see figure 3 or ref. [11] associated with $W \in \frac{1}{2}+\mathbb{Z}$.

\section{7 $\quad \mathbb{P P}^{1}$ at $\theta=\pi$}

The semi-classical approximation must be reliable provided $\mathrm{e}^{-\frac{2 \pi}{g^{2}(\tilde{\ell})}}=\Lambda \tilde{\ell} \lesssim 1$ where $\tilde{\ell}$ is the characteristic distance between two fractional vortex instanton position $\left(\tilde{\ell} \sim\left|a_{i}^{1}-a_{j}^{1}\right|\right)$. So, close fractional vortex instanton positions in Euclidean set-up benefit from asymptotic freedom, just like close quarks do. In principle, we should not use this formula for $\tilde{\ell} \gtrsim \Lambda^{-1}$.

The good news is, the correlation length of the system (at $\theta=0$ ) is given by $\Lambda^{-1}$. After summation over fractional vortex instantons and anti-instantons, the would be long distance divergences are cut-off by the Debye length, and the mass gap can be obtained self-consistently.

Now, this may receive an objection as we change $\theta$. Is it possible that (4.45) is quantitatively and qualitatively correct for any $\theta$ even when correlation length diverges at $\theta=\pi$ ? How seriously should we take the vanishing of the gap at $\theta=\pi$ ?

We encounter exactly the same situation in the leading order semi-classics of the deformed Yang-Mills on small $\mathbb{R}^{3} \times S^{1}$, but there, the gaplessness is lifted at second order (see [48] for details). Here, we will reach to a different conclusion due to renormalization group construction applied to defect theory (4.27). The crucial difference of Coulomb gas in $3 \mathrm{~d}$ and $2 \mathrm{~d}$ is that in the former Coulomb gas is always in the plasma phase (with only one known exception [85]) while in 2d Coulomb gas, the system has a Kosterlitz-Thouless transition, so both gaplessness and gap are possibilities. See [86], page 320 .

This problem is particularly important for anti-ferromagnetic spin chains in 1dimensional spatial lattice. These systems at low energies are described by $2 d \mathbb{C P}^{1}$ nonlinear sigma model. Integer spin is described by a sigma model without theta term and half 
integer spin is described by $\mathbb{C P}^{1}$ with $\theta=\pi, \theta=2 \pi S$. Haldane's conjecture [87] states that integer and half-integer spin chains falls into distinct universality classes, and half-integer spin systems are gapless $(\theta=\pi)$, while the integer spin systems are gapped $(\theta=0)$. See also [88].

Since leading order vertex operators $V_{a}$ produce zero mass gap at $\theta=\pi$, we would like to understand the role of the second order effect of the form:

$$
\left[V_{1} \bar{V}_{2}\right]+\left[V_{2} \bar{V}_{1}\right]+\left[V_{1} \bar{V}_{1}\right]_{ \pm}+\left[V_{2} \bar{V}_{2}\right]_{ \pm}
$$

As we know from resurgence theory, $\left[V_{a} \bar{V}_{a}\right]_{ \pm}$type effects are two-fold ambiguous, and the ambiguous part cancels the renormalon ambiguity in perturbation theory. Their nonambiguous part leads to something extremely interesting. It leads to an anomalous dimension for various important operators.

We would like to determine the amplitude of these events, importantly, their signs compared to vertex operators $V_{a}$. Consider $\mathbb{R}^{2}$ as $\mathbb{R}^{+} \times S^{1}$. In the presence of $\Omega_{F}$ background, we have $V_{a}$ and $\bar{V}_{a}$ events. To write the correlated amplitude, we write an integral over the quasi-zero mode Lefschetz thimble. This gives us:

$$
\begin{aligned}
{\left[V_{1} \bar{V}_{2}\right] } & =\left(-\gamma-\log \left(\frac{A}{g}\right)\right)\left[V_{1}\right]\left[\bar{V}_{2}\right] \\
{\left[V_{1} \bar{V}_{1}\right]_{ \pm} } & =\left(-\gamma-\log \left(\frac{A}{g}\right) \pm \mathrm{i} \pi\right)\left[V_{1}\right]\left[\bar{V}_{1}\right]
\end{aligned}
$$

Again, by resurgent structure [5, 11], ambiguous imaginary parts cancel between Borel resummed perturbation theory and non-perturbative effects. Now, we can write combinations of non-perturbative effects as

$$
\begin{aligned}
& 2 \Upsilon\left[\left(\bar{\psi}_{\mathrm{L}} \psi_{\mathrm{R}}\right)^{2}+\left(\bar{\psi}_{\mathrm{R}} \psi_{\mathrm{L}}\right)^{2}+2\left(\bar{\psi}_{\mathrm{L}} \psi_{\mathrm{R}}\right)\left(\bar{\psi}_{\mathrm{R}} \psi_{\mathrm{L}}\right)\right], \quad \text { where } \\
& 2 \Upsilon=K^{2}\left(-\gamma-\log \left(\frac{A}{g}\right)\right) \mathrm{e}^{-S_{I}}<0
\end{aligned}
$$

The crucial point is that there is a sign difference between the leading order fractional instanton effects and second order correlated fractional instanton effects. ${ }^{17}$ Therefore, we can write the combinations of bion contributions as: $2 \Upsilon\left[\left(\bar{\psi}_{\mathrm{L}} \psi_{\mathrm{R}}\right)^{2}+\left(\bar{\psi}_{\mathrm{R}} \psi_{\mathrm{L}}\right)^{2}-\frac{1}{2} j_{\mu}^{2}\right]$ where $j_{\mu}=\bar{\psi} \gamma_{\mu} \psi$, and $\Upsilon<0$. After understanding these non-trivial facts that arise from

\footnotetext{
${ }^{17}$ This is similar to double-well quantum mechanics. $E_{0}$ is shifted down by $e^{-S_{I}}$ and $E_{1}$ is shifted up by instanton effects, but $E_{0}+E_{1}$ remains the same at leading order in semi-classics. However, at second order, $[I \bar{I}] \sim e^{-2 S_{I}}$ correlated events shifts both $E_{0}$ and $E_{1}$ up by the same amount. In particle on a circle with unique minimum at $\theta=0$, instantons shift the ground state energy down, but both $[I \bar{I}]$ and $[I I]$ shift ground state energy up. So, there is a relative sign between instanton effects and correlated instanton effect in purely bosonic theories. In general, however, the amplitude of the two-events is a subtle and deep issue. If we were to consider $\mathbb{C P}^{1}$ with $\mathrm{n}$ Dirac fermions ( $\mathrm{n}=1$ is supersymmetric theory), it is possible to show that Fugacity $\left[V_{1} \bar{V}_{1}\right]_{ \pm}=$Fugacity $\left[V_{1} \bar{V}_{2}\right] \mathrm{e}^{ \pm \mathrm{in} \pi}$ where $\mathrm{e}^{\mathrm{in} \pi}$ is called hidden topological angle [89] that arise from Lefschetz thimble integration. This extra phase is super-important, and is responsible for the vanishing of the ground state energy in supersymmetric theories, see [89] for details.
} 
resurgence theory, we can proceed with abelian bosonization similar to (4.27). Following almost identical steps with Fradkin's book [86], we obtain

$$
L=\frac{1}{2}(\mathrm{~d} \widetilde{\phi})^{2}+\frac{\Upsilon}{\pi}(\mathrm{d} \widetilde{\phi})^{2}-\left(m^{\prime}\right)^{2}(\theta) \cos (\sqrt{2 \pi} \widetilde{\phi})-4 \Upsilon \cos (2 \sqrt{2 \pi} \widetilde{\phi})
$$

where the first part of the kinetic term is the image of fermion kinetic term, the second part is the image of $j_{\mu}^{2}$, which is sourced by neutral bions $\left[V_{1} \bar{V}_{1}\right]+\left[V_{2} \bar{V}_{2}\right]$, and interaction term arise from charged bions $\left[V_{1} \bar{V}_{2}\right]+\left[V_{2} \bar{V}_{1}\right]$. Note that neutral bion contribution produce a non-linearity which effects the dimensions of the charged bion operator, and this effect will be important.

Setting $\theta=\pi$, and defining $\varphi=\left(1+\frac{2 \Upsilon}{\pi}\right)^{1 / 2} \widetilde{\phi}$ turns the kinetic term to canonical form. Using $\beta^{2}=\frac{2 \pi}{1+\frac{2 Y}{\pi}}$, we can write the action as

$$
L=\frac{1}{2}(\mathrm{~d} \varphi)^{2}-4 \Upsilon \cos (2 \beta \varphi)
$$

Since

$$
\Delta[\cos (2 \beta \varphi)]=\frac{2}{1+\frac{2 \Upsilon}{\pi}}
$$

the charged bion (doubly charged vertex operator), which was exactly marginal at leading order in semi-classical expansion where $\Upsilon=0$, is driven towards irrelevance at second order in semi-classics where $\Upsilon<0$. As a result, the theory at $\theta=\pi$ is gapless.

\subsection{Why is this working? (Improving Polyakov's optimism with RG)}

A source of quantitative error in the formalism is following. The instanton, as we know, has a size moduli, $\rho$. In the classical theory, which is scale invariant, to determine the single instanton contribution, we end up with the integral

$$
Z_{1} \sim V_{2} \int \frac{d \rho}{\rho^{3}} \mathrm{e}^{-\frac{4 \pi}{g^{2}(\rho)}} \sim V_{2} \int \frac{d \rho}{\rho^{3}}(\Lambda \rho)^{N}=V_{2} \Lambda^{N} \int d \rho \rho^{N-3}
$$

which is IR divergent for all $N$. Furthermore, one should not use the one-loop result for the strong scale for $\rho>\rho_{\mathrm{c}} \sim \Lambda^{-1}$ since $g^{2}(\rho)$ becomes large, but at least, at large- $N$ limit, the one-loop result for $\beta$ function becomes exact.

If we go ahead and do a summation over all fractional vortex instanton effects, and construct a grand canonical ensemble of topological defect configurations, we learn that the system induce a finite correlation length $\xi=m^{-1} \sim \Lambda^{-1}$. This immediately tells us that one should not extrapolate (4.57) naively. Instead, we interpret the Debye length as a cut-off, replacing

$$
\int d \rho \rho^{N-3} \mapsto \int d \rho \rho^{N-3} \mathrm{e}^{-m \rho}
$$

This removes the IR divergent bad domain $\rho>\xi$ from integration, see figure 5. Since $\rho_{c} \sim \xi$, it is impossible to do qualitative error in this way, and the infrared theory for $\mathbb{C P}^{N-1}$ which 


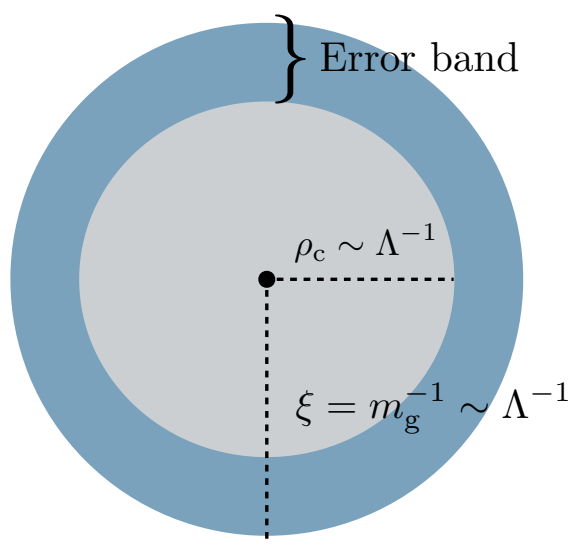

Figure 5. This diagram depicts a source of quantitative error. We use one-loop result for strong scale, which should be reliable for $\tilde{l} \lesssim \rho_{c} \sim \Lambda^{-1}$ and unreliable for $\tilde{l} \gtrsim \rho_{c}$, where it leads to an IR divergence. At the same time, the proliferation of fractional vortex instantons with fugacity $\mathrm{e}^{-S_{I} / N}$ generates a finite correlation length $\xi \sim \Lambda^{-1}$. Because of the Debye screening, this cuts-off the interaction between fractional vortex instantons at distances larger than $\xi \sim \rho_{c}$, essentially removing the bad zone from integration. Depending on the numerical values of $\xi \sim \rho_{c}$, there may be a band which induce quantitative error. We then use RG arguments to find relevant operators at $\theta=0$. Since RG is independent of $\theta$, we can extrapolate our effective theory to arbitrary $\theta$. In $\mathbb{C P}^{1}$, this gives us a gapless theory at $\theta=\pi$. Despite the fact that "naive" error band becomes infinite, this result is reliable because the determiner of error is $\theta=0$. Therefore, both gap and gaplessness are robust qualitative predictions of the formalism.

captures low lying modes is described by the mass deformed $\mathrm{SU}(N)_{1}$ WZW (4.41), where mass deformation is governed by $m \sim \mu \mathrm{e}^{-S_{I} / N}=\Lambda$.

The logic of the previous paragraph follows Polyakov, [66], section 6.1. However, in the important case of $N=2$, the rationale of Polyakov breaks down around $\theta \sim \pi$ where correlation length diverges as $\xi(\theta)=\Lambda^{-1} \frac{1}{|\cos (\theta / 2)|^{2 / 3}}$.

Should we not trust this result as the bad domain $\rho \in\left[\Lambda^{-1}, \infty\right)$ resurfaces in the integration (4.58)? Is this prediction unreliable? Our claim is otherwise. We should trust it as much as we trust the $\theta=0$ results. We will prove this by improving Polyakov's logic with renormalization group.

The $\theta=0$ and $\theta \neq 0$ are not independent theories, we are just adding a topological theta angle to the theory. If we did everything right at $\theta=0$ and constructed the correct long distance theory based on renormalization group of topological defects, why should it fail by turning on a topological angle at $\theta=\pi$ ?

Indeed, at $\theta=0$, we showed that the vertex operators $\mathrm{e}^{\mathrm{i} l \beta \varphi}$ have dimensions

$$
\Delta_{l} \equiv \Delta\left[\mathrm{e}^{\mathrm{i} l \beta \varphi}\right]=\frac{l^{2} \beta^{2}}{4 \pi}=\frac{1}{2\left(1+\frac{2 \Upsilon}{\pi}\right)} l^{2},
$$

Therefore, unit charge vertex operator has dimension $\sim \frac{1}{2}$ and is highly relevant, $l \geq 3$ are irrelevant, and $l=2$ vertex operator is marginally irrelevant. This construction is certainly 
valid at $\theta=0$ where our EFT is self-consistent. The scaling dimensions are independent of $\theta$ angle. If we construct effective theory correctly at $\theta=0$, turning on $\theta$ will not change that fact, and despite the fact that $\xi(\theta) \gg \rho_{c}$, we must obtain the correct results at arbitrary $\theta$.

The theory does not become gapless at $\theta=\pi$ because $\mathrm{e}^{\mathrm{i} \beta \varphi}$ becomes irrelevant, to the contrary, its is extremely relevant. The theory becomes gapless because of the destructive topological interference between $V_{1}=\mathrm{e}^{-\frac{S_{I}}{2}} \mathrm{e}^{\mathrm{i} \beta \varphi+\mathrm{i} \frac{\theta}{2}}$ and $\bar{V}_{2}=\mathrm{e}^{-\frac{S_{I}}{2}} \mathrm{e}^{\mathrm{i} \beta \varphi-\mathrm{i} \frac{\theta}{2}}$, and the fact that the $l=2$ vertex operator is marginally irrelevant.

For general $\mathbb{C P}^{N-1}, N \geq 3$ theories, we are even safer in our construction. There are only three types of relevant operators in the renormalization group of vertex opetators, (see (4.37) for a full list)

$$
\begin{aligned}
\operatorname{dim}\left(V_{a}\right) & =\left(1-\frac{1}{N}\right) \\
\operatorname{dim}\left(V_{a} V_{b}\right) & =2\left(1-\frac{2}{N}\right), \quad \operatorname{dim}\left(V_{a} \bar{V}_{b}\right)=2 \quad a \neq b,
\end{aligned}
$$

and all the rest is irrelevant. Since $\operatorname{dim} \sim 2$ for $\left[V_{a} \bar{V}_{b}\right]$ and $\left[V_{a} V_{b}\right]$ operators, they do not alter the conclusions of $\operatorname{dim} \sim 1 V_{a}$ operators, we can ignore their effects accepting quantitative error. Since we are after the more modest goal of determining the existence of gap, we hope this is acceptable. Therefore, our demonstration of mass gap for $N \geq 3$ theories on $\mathbb{R}^{2}$ is on a robust footing.

Mass deformed WZW interpretation. Of course, the above statements translate to non-abelian bosonization language nicely (4.41). The massive $U$ field in the mass deformed $\mathrm{SU}(N)_{1}$ WZW describe the massive adjoint $\bar{z}_{a}(x) z^{b}(x)$ field and singlet (slightly heavier) $\bar{z}_{a}(x) \mathrm{e}^{\mathrm{i} \int_{x}^{y} a} z^{a}(y)$ field in the spectrum, $\left(N^{2}-1\right) \oplus 1$. In the large- $N$ limit, $\operatorname{tr} U$ mass deformation has dimension $\Delta \sim 1$. Double trace operators $|\operatorname{tr} U|^{2},(\operatorname{tr} U)^{2}$ have $\Delta \sim 2$. These describe the low lying spectrum of the $\mathbb{C P}^{N-1}, N \geq 3$ model, an adjoint and a singlet whose masses are at the strong scale of the theory.

For the $N=2$ case, we need to pay more attention. Let us write

$$
U_{a b} \sim \mathrm{e}^{\mathrm{i} \sqrt{\frac{4 \pi}{N}} \widetilde{\phi}} g_{a b}
$$

in (4.41). In this case, at $\theta=0$, we have a massive triplet and slightly heavier singlet. In fact, as $\theta$ goes from 0 to $\pi$, the gap between lowest lying triplets and singlet widens, and it becomes fully justified to integrate out the heavy $\widetilde{\phi}$ mode. In this case, in the IR, $\Delta[\operatorname{tr} g]=\frac{1}{2}$, but this operator disappears from deformed WZW action due to topological interference induced by $\theta$ term. A possible mass term may be generated by the double trace operator, $|\operatorname{tr} g|^{2}$. But this operator has $\Delta\left[|\operatorname{tr} g|^{2}\right]>2$ (4.56) and is marginally irrelevant. Therefore, at $\theta=\pi$, we obtain a gapless triplet and a massive singlet. (Same conclusion for $N=2$ theory are also reached by Affleck in ref. [90] by different means.) 


\section{What are we summing over in semiclassics in dYM theory on $\mathbb{R}^{3} \times S^{1}$ ?}

Deformed Yang-Mills theory is center-symmetry preserving double-trace deformation of the Yang-Mills action on small $\mathbb{R}^{3} \times S^{1}[14,15]$. In the sense of gauge invariant order parameters, it is continuously connected to pure YM on $\mathbb{R}^{4}$. The deformation allows a calculable (semi-classical) regime in which one can study non-perturbative properties such as confinement, $\theta$ angle dependence, mass gap generation. See $[12-14,50,91-107] .{ }^{18}$ There is by now compelling evidence that deformed $\mathrm{YM}, \mathrm{QCD}(\mathrm{F})$ with $\Omega_{F}$ twist, $\mathrm{QCD}(\mathrm{adj})$ and a number of other theories on small $\mathbb{R}^{3} \times S_{L}^{1}$ regime are continuously connected to the corresponding theories on $\mathbb{R}^{4}$. Recently, $[111,112]$ showed by numerical lattice simulations that topological susceptibility at small $\mathbb{R}^{3} \times S_{L}^{1}$ dYM theory is identical to the one of pure YM theory on large $T^{4} \sim \mathbb{R}^{4}$. It is important to recall that in these theories, the $S_{L}^{1}$ circle never has a thermal interpretation in the standard sense [12].

It should be noted that there also had been important body of work on $T^{3} \times \mathbb{R}$ by using 't Hooft twisted boundary conditions, pioneered by Gonzalez-Arroyo, Garcia-Perez and van Baal [31-35, 41, 113-120]. (See also [29, 121, 122] for other uses of twisted boundary conditions.) These works, in the small $T^{3}$ regime, aimed to construct a semi-classical description of the vacuum and confinement mechanism based on 't Hooft's fractional topological charge $W=1 / N$ and action $S=S_{I} / N$ configurations. As we emphasized multiple times, 't Hooft's original solutions on $T^{4}$ are constant (space-time independent) and to a certain degree, (apart from its high value in showing that action $S=S_{I} / N$ configuration do exist), are fairly uninteresting. The biggest stumbling block in this program had been the lack of exact analytic non-trivial (spacetime dependent) solution with $S=S_{I} / N$. However, these authors were able to demonstrate the existence of non-trivial BPS $S=S_{I} / N$ (time dependent) configurations by using lattice techniques, leaving no doubt for their existence, though these works are not sufficiently appreciated. Some semi-classical dynamics due to fractional instantons is understood. (See [33] and also [41] for a review.) It should be noted that these works are prior to the explicit analytical solutions of $W=1$ non-trivial holonomy calorons and their fractionization to $W=1 / N$ constituents. And possible connections between fractional instantons and monopole instantons are partly discussed in $[115,120]$, but it is not concluded that monopole-instantons live in $\operatorname{PSU}(N)$ bundle, a missing link between the two that we filled up in the present work.

Our analysis of Yang-Mills theory on $M_{4}$ can be viewed in some sense putting the analysis of gauge theories on $T^{3} \times \mathbb{R}$ and $\mathbb{R}^{3} \times S^{1}$ in complete agreement. In particular, we are able to do so by proving that the monopole-instanton are actually exact solutions in the $\operatorname{PSU}(N)$ bundle. This fulfills a (numerically substantiated) key assumption in the analysis of Gonzalez-Arroyo et al. Then, we show that by coupling a TQFT to gauge theory, that the configurations with action $1 / N$ are also present in the strong coupling domain.

Returning back to $\mathbb{R}^{3} \times S^{1}$ studies, despite the fact that the long distance theory of

\footnotetext{
${ }^{18}$ Modeling of Yang-Mills vacuum outside its region of validity of semi-classics is described in [108-110]. The construction in this paper provides some retrospective rationale for this study. In particular, the coupling of YM to $\mathbb{Z}_{N}$ TQFT proves that fractional topological charge $W=1 / N$ configurations is there even at strong coupling of pure YM theory.
} 
the theories on $\mathbb{R}^{3} \times S^{1}$ reduce to $3 \mathrm{~d}$ theory, the microscopic theory is $4 \mathrm{~d}$. The deformed YM and QCD theories on $\mathbb{R}^{3} \times S_{L}^{1}$ are closer in all respect to their decompactifcation limit on $\mathbb{R}^{4}$ than the theories on $\mathbb{R}^{3}$ limit. ${ }^{19}$ For example, these theories have theta angle parameters, multi-branch structure, mass gap determined by $4 \mathrm{~d}$ strong scale, global ABJ anomalies. Very often, they also have the same global and mixed anomalies as the $4 \mathrm{~d}$ theories [52, 85, 123].

Below, we would like to determine in detail the configurations that contribute to partition function of deformed Yang-Mills in the semi-classical domain in the same spirit as our simple quantum mechanical $T_{N}$ example. But before that, we would like to provide a quick review of the deformed theory and set the notation.

\section{Quick overview of deformed Yang-Mills: remembrance of things past.}

- In pure Yang-Mills theory, center symmetry is broken at small circle size (hightemperature) on $\mathbb{R}^{3} \times S^{1}$ [124]. Center-symmetry can be stabilized at small- $L$ by the addition of double-trace operator $\Delta \mathcal{L}=\sum_{k=1}^{\left\lfloor\frac{N}{2}\right\rfloor}\left|\operatorname{tr} U_{3}^{k}\right|^{2}$ where $U_{3}$ denotes Polyakov loop along compact $x_{3} \sim x_{3}+L$ circle. ${ }^{20}$ Despite the fact that this term looks nonlocal, it can arise from local action, e.g. Yang-Mills + (massless or massive) adjoint fermions at small $S^{1}$ with periodic boundary conditions induce the center stabilization dynamically. See $[12,14,15]$ for details.

- The minimum of the holonomy potential is given by $\mathrm{e}^{\mathrm{i} \phi_{\star}}=\left(1, \omega, \omega^{2}, \ldots, \omega^{N-1}\right)$ up to Weyl permutations, where $\left(\mathrm{e}^{\mathrm{i} \phi_{\star}}\right)_{i}=\left(U_{3}\right)_{i i}$. This forces the dynamics to abelianize at small- $L$, and at long distances, the theory is described by maximal abelian subgroup $\mathrm{SU}(N) \rightarrow \mathrm{U}(1)^{N-1}$, just in terms of photons. Off-diagonal gluons gets masses $\geq 2 \pi / N L$.

- There are multiple useful basis to describe the IR physics. The diagonal components of gauge fields and compact scalar can be written as $\boldsymbol{a}=\sum_{i=1}^{N-1} a_{i} \boldsymbol{\alpha}_{i}$ where $a_{i}$ are canonically normalized gauge fields and $\boldsymbol{\phi}=\sum_{i=1}^{N-1} \phi_{i} \boldsymbol{\alpha}_{i}=\left(\phi_{1}, \phi_{2}-\phi_{1}, \ldots,-\phi_{N-1}\right)$. The periodicity of $\boldsymbol{\phi}$ is governed by the root lattice, $\phi \sim \phi+2 \pi \boldsymbol{\alpha}_{i}, \boldsymbol{\alpha}_{i} \in \Gamma_{r}$,

$$
\phi \in \frac{\mathbb{R}^{N-1}}{2 \pi \Gamma_{r}}
$$

hence, $\phi_{i}$ are periodic variable with period $2 \pi . \phi_{\star}$ is the center of Weyl chamber where dynamics completely abelianize.

- We can perform abelian duality [66] and express the free $3 \mathrm{~d}$ action as

$$
S_{\text {eff }}=\int\left(\frac{1}{2 g^{2} L}|\mathrm{~d} \phi|^{2}+\frac{g^{2}}{8 \pi^{2} L}\left|\mathrm{~d} \boldsymbol{\sigma}-\frac{\theta}{2 \pi} \mathrm{d} \phi\right|^{2}\right)
$$

\footnotetext{
${ }^{19}$ In fact, even at small- $L$, where $L \Lambda \sim O\left(N^{0}\right)$, taking $N \rightarrow \infty$, these theories are equivalent to the theories on $\mathbb{R}^{4}$. In certain sense, effective circle size is $L_{\mathrm{eff}}=N L$ and at $N=\infty$, for the neutral sector observables (this is the sector singlet under zero-form part of center symmetry), it is fair to interpret this as if there is no compactification. This is called large- $N$ volume independence.

${ }^{20}$ We refer to non-compact directions as $x_{1}, x_{2}, x_{4}$. This convention is more useful when we describe the same set-up with the insertion of 't Hooft flux.
} 
In most theories, we can also forget about $\phi$ as it is gapped by holonomy potential, and can be dropped in long distance effective field theory. The dual photon field $\boldsymbol{\sigma}$ has periodicity determined by weight lattice, $\boldsymbol{\sigma} \sim \boldsymbol{\sigma}+2 \pi \boldsymbol{\mu}_{i}, \boldsymbol{\mu}_{i} \in \Gamma_{w}$.

$$
\boldsymbol{\sigma} \in \frac{\mathbb{R}^{N-1}}{2 \pi \Gamma_{w}}
$$

A useful basis for our purpose is $\boldsymbol{\sigma}=\sum_{i=1}^{N-1} \sigma_{i} \boldsymbol{\mu}_{i}$, hence $\sigma_{i} \sim \sigma_{i}+2 \pi$, and $\sigma_{i}$ are $2 \pi$-periodic scalars.

- There are $N$ types of the fundamental monopole instantons, and the monopole operators are given by

$$
\mathcal{M}_{a}(x)=\mathrm{e}^{-S_{I} / N} \mathrm{e}^{\mathrm{i} \boldsymbol{\alpha}_{a} \cdot \boldsymbol{\sigma}(x)} \mathrm{e}^{\mathrm{i} \theta / N} \quad(a=1, \ldots, N) .
$$

$N-1$ of these monopoles are the regular ones associated with $\mathrm{SU}(N) \rightarrow$ $\mathrm{U}(1)^{N-1}$ adjoint Higgsing. The monopole associated with the affine root $\boldsymbol{\alpha}_{N}=$ $-\sum_{a=1}^{N-1} \boldsymbol{\alpha}_{a}$ [125-129] has the same action with the rest of the simple roots due to compactness of the adjoint scalar or equivalently, due to the fact that Polyakov loop acts as a group valued field at a center-symmetric background. See also [130].

- The sum over all monopole instantons generates an effective potential. The grand canonical ensemble of the monopole gives the partition function:

$$
Z=\int \mathcal{D} \boldsymbol{\sigma} \exp \left[-\left(\int \frac{g^{2}}{8 \pi^{2} L}|\mathrm{~d} \boldsymbol{\sigma}|^{2}-2 K \mathrm{e}^{-S_{I} / N} \sum_{a=1}^{N} \cos \left(\boldsymbol{\alpha}_{a} \cdot \boldsymbol{\sigma}+\frac{\theta}{N}\right)\right)\right]
$$

which leads to a non-perturbative mass gap, finite string tension with $\mathrm{N}$-ality $k \lesssim$ $N / 2, N$-branched vacua, and CP breaking at $\theta=\pi$.

- The effective field theory description in terms monopole-instanton and bion local fields is based on the parametric scale separation between the dual photon and $W$-boson, and is valid provided $(\Lambda L N) \lesssim 1$. However, EFTs can be used to determine some observables, but not all observables. For example, despite the fact that EFT (5.5) can produce mass gap, string tension with $N$-ality $k \lesssim N / 2[12,14,70]$, exact value of chiral condensate in $\mathcal{N}=1 \mathrm{SYM}$ [131], it cannot produce string tensions with $N$-ality $k \gtrsim N / 2$ correctly. Reproducing those require the inclusion of $W$-bosons, which are present in microscopic theory, but not in EFT. We will dwell onto these subtleties a bit more precisely in the conclusion section.

\subsection{Mini-space formalism: global constraints in monopole-instanton sums}

A number of issues come to mind with this construction and has been raised over the years.

Configurations contributing to partition function. One is the appearance of $\theta / N$ in the monopole operator (5.4) and effective action (5.5) that arise from a monopole-instanton with topological charge $Q=\frac{1}{N}$. Yet, if we consider the $\mathrm{SU}(N)$ theory on orientable 4manifolds, it is well-known that the topological charge is quantized in integer units $W \in \mathbb{Z}$. 
Therefore, the configurations that contribute to the partition function must have a theta dependence of the form $\mathrm{e}^{i W \theta}$. How is this compatible with the appearance of $\theta / N$ in the monopole operators and effective action?

If we compactify the theory on small $\mathbb{R}^{3} \times S_{L}^{1}$ down to quantum mechanics, $T^{2} \times$ $S_{\beta}^{1} \times S_{L}^{1}$, then, in the path integral representation of the partition function $Z(\beta)=$ $\operatorname{tr} \exp \left[-\beta H_{T^{2} \times S_{L}^{1}}\right]$, we are supposed to sum over fields that obey periodic boundary conditions. Yet, the presence of a monopole implies that the magnetic flux piercing $T^{2}$ $\boldsymbol{\Phi}_{\text {mag }}(\tau)=\int_{T^{2}} \boldsymbol{B}$ changes by

$$
\boldsymbol{\Phi}_{\mathrm{mag}}(\beta)-\boldsymbol{\Phi}_{\mathrm{mag}}(0)=\left.\left(\int_{T^{2}} \boldsymbol{B}\right)\right|_{\tau=0} ^{\tau=\beta}=\frac{2 \pi}{g} \boldsymbol{\alpha}_{a}, \quad a=1, \ldots, N .
$$

Such configurations are present in the theory, but just like our quantum mechanical example, they should not contribute to partition function, which is a sum over periodic paths. But of course, despite the fact that these contribution are absent in the sum, most of the non-perturbative low energy phenomena are sourced by them, again just like our QM example.

The image of the issue raised on $T^{2} \times S_{\beta}^{1} \times S_{L}^{1}$ for the infinite volume theory can be simply described as follows. The magnetic flux at infinity on $\mathbb{R}^{3} \times S^{1}$ is a choice of boundary condition. If we choose the flux at infinity to be zero, the net flux from all sources must vanish by Gauss's law, the only configuration that can contribute to the partition function must satisfy magnetic neutrality at infinity $\int_{S_{\infty}^{2}} \boldsymbol{B}_{\text {total }}=0$.

Below, we show that the master partition function (5.5) is aware of these issues and cleverly takes these constraints into account, as mentioned in passing in [132]. We prove in detail that magnetic charge neutrality comes naturally from (5.5). Furthermore, as a bonus, once magnetic charge neutrality emerges, the topological charge of corresponding configurations become integer quantized. (Opposite is not true, configurations with integer winding number need not be magnetically neutral.) We also note that the points about global neutrality are also emphasized in $[133,134]$, though we disagree with the naming of monopole-instanton as dyon, since it is not an eigenstate of electric charge operator.

Minispace formalism. The path integration over the fields $\int \mathcal{D} \boldsymbol{\sigma} \mathrm{e}^{-S}$ has a zero mode part, the integration over the space of constant (space-time) independent $\boldsymbol{\sigma}$. In this subspace, the measure reduce to an ordinary integral over the fundamental cell of $\boldsymbol{\sigma}$ field given in (5.3),

$$
\int_{\sigma \in \frac{\mathbb{R}^{N-1}}{2 \pi \Gamma w}}[d \boldsymbol{\sigma}] \equiv\left(\prod_{i=1}^{N-1} \int_{0}^{2 \pi} d \sigma_{i}\right)
$$

The action over the constant modes reduces to $s=-2 V_{3} K \mathrm{e}^{-S_{I} / N} \sum_{a=1}^{N} \cos \left(\boldsymbol{\alpha}_{a} \cdot \boldsymbol{\sigma}+\frac{\theta}{N}\right)$. So, the zero mode integration becomes ( $\operatorname{set} \xi=V_{3} K \mathrm{e}^{-S_{I} / N}$ )

$$
\begin{aligned}
z(\theta) & =\int_{\text {cell }}[d \boldsymbol{\sigma}] \mathrm{e}^{-s} \\
& =\left(\prod_{i=1}^{N-1} \int_{0}^{2 \pi} d \sigma_{i}\right) \prod_{a=1}^{N} \mathrm{e}^{\xi \mathrm{e}^{\mathrm{i}\left(\boldsymbol{\alpha}_{a} \cdot \boldsymbol{\sigma}+\frac{\theta}{N}\right)}} \mathrm{e}^{\xi \mathrm{e}^{-\mathrm{i}\left(\boldsymbol{\alpha}_{a} \cdot \boldsymbol{\sigma}+\frac{\theta}{N}\right)}}
\end{aligned}
$$


We call $s(\boldsymbol{\sigma})$ mini-space action and $z(\theta)$ mini-space partition function. Using the good basis $\boldsymbol{\sigma}=\sum_{i=1}^{N-1} \sigma_{i} \boldsymbol{\mu}_{i}$, we have $\boldsymbol{\alpha}_{i} \cdot \boldsymbol{\sigma}=\sigma_{i}, n=1, \ldots, N-1$ and $\boldsymbol{\alpha}_{N} \cdot \boldsymbol{\sigma}=-\left(\sigma_{1}+\ldots+\sigma_{N-1}\right)$, which allows us to easily perform the integration.

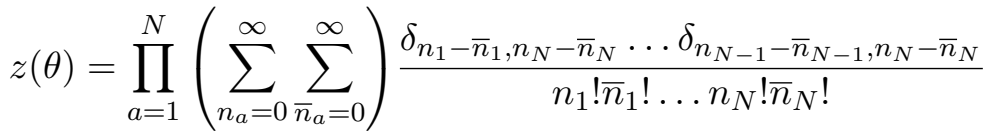

$$
\begin{aligned}
& \xi^{n_{1}+\ldots+n_{N}+\bar{n}_{1}+\ldots+\bar{n}_{N}} \mathrm{e}^{i \frac{\theta}{N}\left(n_{1}+\ldots+n_{N}-\left(\bar{n}_{1}+\ldots+\bar{n}_{N}\right)\right)}
\end{aligned}
$$

where $N-1$ constraints comes from the integration over the $N-1$ independent $\sigma_{i}$ field. The solution of the constraints is

$$
n_{1}-\bar{n}_{1}=n_{2}-\bar{n}_{2}=\ldots=n_{N}-\bar{n}_{N}=W
$$

where $W \in \mathbb{Z}$. This enforces magnetic neutrality, i.e, magnetic flux at infinity is zero. This constraint tells us that $n_{a}-\bar{n}_{a}$ is independent of $a$. Clearly, if $n_{a}-\bar{n}_{a}$ is zero, both magnetic neutrality and topological neutrality are guaranteed. But what if $n_{a}-\bar{n}_{a}=W, \forall a$, an $a$-independent excess of monopoles over anti-monopoles? In that case, magnetic neutrality is guaranteed thanks to the fact that $\sum_{a=1}^{N} \boldsymbol{\alpha}_{a}=0$. Furthermore, when this condition is satisfied, the corresponding configuration has an integer topological charge given by $W \in \mathbb{Z}$ ! Therefore, the mini-space partition function can be written as

$$
z(\theta)=\sum_{W \in \mathbb{Z}}\left(\sum_{\bar{n}_{1}=0}^{\infty} \ldots \sum_{\bar{n}_{N}=0}^{\infty}\right) \frac{1}{\left(\bar{n}_{1}+W\right) ! \bar{n}_{1} ! \ldots\left(\bar{n}_{N}+W\right) ! \bar{n}_{N} !} \xi^{2 \bar{n}_{1}+\ldots+2 \bar{n}_{N}+N W} \mathrm{e}^{i W \theta}
$$

This elegant formula tells us many interesting things:

- The configurations that contribute to the partition function have fractional actions and integer topological charges:

$$
\begin{aligned}
& S=\frac{S_{I}}{N}\left(2 \bar{n}_{1}+\ldots+2 \bar{n}_{N}\right)+S_{I}|W| \in S_{I}\left(\frac{2}{N}|k|+|W|\right), \quad k, W \in \mathbb{Z} \\
& Q=W \in \mathbb{Z}
\end{aligned}
$$

just like our simple quantum mechanical $T_{N}$ model.

- Despite the fact that the monopole instantons have fractional topological charge, only their magnetically neutral combinations contribute to the sum. Magnetic neutrality enforces the quantization of topological charge as well. This satisfies the boundary condition at infinity on $S_{\infty}^{2}$.

- This construction also holds for any sufficiently large $M_{3}$ times small $S_{L}^{1}$. We can think $\mathbb{R}^{3}$ as the infinite volume limit of $M_{3}$.

We can decompose mini-space partition function into its Fourier modes $z(\theta)=$ $\sum_{W \in \mathbb{Z}} z_{W} \mathrm{e}^{\mathrm{i} W \theta}$, and comparing with (5.11); we learn that

$$
\begin{aligned}
z_{W} & =\left(\sum_{\bar{n}_{1}=0}^{\infty} \ldots \sum_{\bar{n}_{N}=0}^{\infty}\right) \frac{1}{\left(\bar{n}_{1}+W\right) ! \bar{n}_{1} ! \ldots\left(n_{N}+W\right) ! n_{N} !} \xi^{2 \bar{n}_{1}+\ldots+2 \bar{n}_{N}+N W} \\
& =\left[I_{W}(2 \xi)\right]^{N}
\end{aligned}
$$


where $I_{W}(2 \xi)$ is modified Bessel function. $z_{W}$ carries the information of all configurations (within the dilute monopole gas approximation) contributing to the topological sector with charge $W \in \mathbb{Z}$.

Tunneling events that contribute to the partition function are the transitions between pure gauge configurations which differ in the winding number $W$ only, just like regular instantons on $\mathbb{R}^{4}$. Net magnetic flux is zero at infinity, but this does not mean that the corresponding configurations are boring. Instead, each contributing configuration has substructure, $n_{a}-\bar{n}_{a}=W, \forall a$ monopole-instantons.

\subsection{Vacuum energy density and multi-branch structure}

A final remark is on the $N$-branched vacuum structure of deformed Yang-Mills theory. Monopole induced potential has $N$ extrema within the fundamental domain of $\boldsymbol{\sigma}$ field given in (5.3). These are:

$$
\boldsymbol{\sigma}_{k}=\frac{2 \pi}{N} k \boldsymbol{\rho} \equiv \frac{2 \pi}{N} k \sum_{i=1}^{N-1} \boldsymbol{\mu}_{i},
$$

with $k=0,1, \ldots, N-1$ and $\rho$ is the Weyl vector. Approximately $\frac{N}{2}$ of these branches are meta-stable for a given value of $\theta$ [135]. As $\theta$ is varied, the set of meta-stable vacua changes. From this, we can deduce the vacuum energy densities as

$$
\mathcal{E}_{k}=-2 N K \mathrm{e}^{-S_{I} / N} \cos \left(\frac{\theta+2 \pi k}{N}\right) .
$$

As a result, we can write the partition function for the theory defined on a 3-manifold as

$$
Z(\theta)=\sum_{k=0}^{N-1} \mathrm{e}^{2 N V_{3} K \mathrm{e}^{-S_{I} / N} \cos \left(\frac{\theta+2 \pi k}{N}\right)} \equiv \sum_{k=0}^{N-1} \mathrm{e}^{2 N \xi \cos \left(\frac{\theta+2 \pi k}{N}\right)}
$$

which can be converted into a fractional instanton sum:

$$
\begin{aligned}
Z(\theta) & =N \sum_{W \in \mathbb{Z}} \sum_{n=0}^{\infty} \sum_{\bar{n}=0}^{\infty} \frac{1}{n !} \frac{1}{\bar{n} !}\left(N \xi \mathrm{e}^{\mathrm{i} \frac{\theta}{N}}\right)^{n}\left(N \xi \mathrm{e}^{-\mathrm{i} \frac{\theta}{N}}\right)^{\bar{n}} \delta_{n-\bar{n}-W N, 0} \\
& =N \sum_{W \in \mathbb{Z}} \sum_{\bar{n}=0}^{\infty} \frac{1}{(n+W N) !} \frac{1}{\bar{n} !}(N \xi)^{2 \bar{n}+W N} \mathrm{e}^{\mathrm{i} W \theta} \\
& =N \sum_{W \in \mathbb{Z}} I_{W N}(2 \xi N) \mathrm{e}^{\mathrm{i} W \theta}
\end{aligned}
$$

In (5.17), quantization of topological charge emerges as a result of the constraint, $\delta_{n-\bar{n}-W N, 0}$. In this formula, there is no information about magnetic charges of the individual monopole instantons. That information is washed away by the choice of the vacuum branch in (5.16). At the $k^{\text {th }}$ branch, all monopole operators with non-zero adjoint charge (also called GNO charge) but zero 't Hooft charge acquire the same vev, $\left\langle\mathrm{e}^{\mathrm{i} \boldsymbol{\alpha}_{a} \cdot \boldsymbol{\sigma}}\right\rangle=\mathrm{e}^{\mathrm{i} 2 \pi k} \frac{135]}{N}$ and the information about individual magnetic charges $\boldsymbol{\alpha}_{a}$ of $N$ types of monopoles is lost. In this sense, (5.11) has more information than (5.17). It knows that $n_{a}-\bar{n}_{a}=W \quad \forall a$. This naturally implies $\sum_{a=1}^{N}\left(n_{a}-\bar{n}_{a}\right) \equiv n-\bar{n}=W N$. 


\section{Coupling Yang-Mills theory to $\mathbb{Z}_{N}$ TQFT}

The Lagrangian of $\mathrm{SU}(N)$ Yang-Mills theory is

$$
\mathcal{L}_{\mathrm{YM}}=\frac{1}{2 g_{\mathrm{YM}}^{2}} \int \operatorname{tr}[F \wedge \star F]+\frac{\mathrm{i} \theta_{\mathrm{YM}}}{8 \pi^{2}} \int \operatorname{tr}[F \wedge F]
$$

where the second term is properly quantized topological term:

$$
Q=\frac{1}{8 \pi^{2}} \int \operatorname{tr}[F \wedge F] \in \mathbb{Z}
$$

$\mathrm{SU}(N)$ YM theory possess a $\mathbb{Z}_{N}^{[1]}$ electric one-form symmetry, which acts on Wilson line operators.

To probe the topological configurations in YM theory, we follow the same method as in our $T_{N}$ quantum mechanics example. We turn on a background gauge field for the $\mathbb{Z}_{N}^{[1]}$ 1-form symmetry [2, 136], introducing pair of $\mathrm{U}(1)$ 2-form and 1-form gauge fields $\left(B^{(2)}, B^{(1)}\right)$ satisfying

$$
N B^{(2)}=\mathrm{d} B^{(1)}, \quad N \int B^{(2)}=\int \mathrm{d} B^{(1)}=2 \pi \mathbb{Z}
$$

The action corresponding to the $\mathbb{Z}_{N}$ topological gauge theory is given by

$$
Z_{\text {top }, p}=\int \mathcal{D} B^{(2)} \mathcal{D} B^{(1)} \mathcal{D} C^{(2)} \mathrm{e}^{\mathrm{i} \int C^{(2)} \wedge\left(N B^{(2)}-\mathrm{d} B^{(1)}\right)+\mathrm{i} p \frac{N}{4 \pi} \int B^{(2)} \wedge B^{(2)}}
$$

where $C^{(2)}$ is Lagrange multiplier, and $p$ is the discrete theta angle.

To couple the $\mathrm{SU}(N)$ YM theory to the background gauge field $B^{(2)}$, we promote the $\mathrm{SU}(N)$ gauge field $a$ into a $\mathrm{U}(N)$ gauge field $\tilde{a}$. The $\mathrm{U}(N)$ gauge field is related to dynamical $\mathrm{SU}(N)$ gauge field $a$ locally as

$$
\widetilde{a}=a+\frac{1}{N} B^{(1)} .
$$

To gauge $\mathbb{Z}_{N}^{[1]}$, we introduce gauge invariance under the one-form gauge transformation,

$$
B^{(2)} \mapsto B^{(2)}+\mathrm{d} \Lambda^{(1)}, \quad B^{(1)} \mapsto B^{(1)}+N \Lambda^{(1)},
$$

and hence,

$$
\widetilde{a} \mapsto \widetilde{a}+\Lambda^{(1)}, \quad \widetilde{F} \mapsto \widetilde{F}+\mathrm{d} \Lambda^{(1)}
$$

In writing $\mathrm{SU}(N)$ theory in a $\mathbb{Z}_{N}$ TQFT (equivalently 't Hooft flux) background, we have to replace $\mathrm{SU}(N)$ field strength with the gauge-invariant combination of the $\mathrm{U}(N)$ field strength $\widetilde{F}=F(\widetilde{a})$ and $B^{(2)}$ :

$$
F(a) \Rightarrow \widetilde{F}-B^{(2)}
$$

The action of the $\mathrm{SU}(N)$ theory in the $B^{(2)}$ background can be written as:

$S\left[B^{(2)}, B^{(1)}, \widetilde{a}\right]=\frac{1}{2 g_{\mathrm{YM}}^{2}} \int \operatorname{tr}\left[\left(\widetilde{F}-B^{(2)}\right) \wedge \star\left(\widetilde{F}-B^{(2)}\right)\right]+\frac{\mathrm{i} \theta_{\mathrm{YM}}}{8 \pi^{2}} \int \operatorname{tr}\left[\left(\widetilde{F}-B^{(2)}\right) \wedge\left(\widetilde{F}-B^{(2)}\right)\right]$ 
If we further wish to obtain the partition function of the $\left(\mathrm{SU}(N) / \mathbb{Z}_{N}\right)_{p}$ gauge theory, then, we have to sum over all $B^{(2)}, \mathbb{Z}_{N}$ valued 2-form gauge fields. Namely,

$$
Z_{\left(\mathrm{SU}(N) / \mathbb{Z}_{N}\right)_{p}}=\int \mathcal{D} B^{(2)} \mathcal{D} B^{(1)} \mathcal{D} C^{(2)} \mathcal{D} \widetilde{a} \mathrm{e}^{\mathrm{i} \int C^{(2)} \wedge\left(N B^{(2)}-\mathrm{d} B^{(1)}\right)+\mathrm{i} p \frac{N}{4 \pi} \int B^{(2)} \wedge B^{(2)}} \mathrm{e}^{-S\left[B^{(2)}, B^{(1)}, \widetilde{a}\right]}
$$

where

$$
\frac{N}{8 \pi^{2}} \int B^{(2)} \wedge B^{(2)} \in \frac{1}{N} \mathbb{Z}
$$

is the $\mathbb{Z}_{N}$ 't Hooft flux. This partition function is the counter-part of our simple quantum mechanical example (2.35). For other background fields that one can turn on in Yang-Mills theory, see [137].

In $\mathrm{SU}(N)$ theory, topological charge is quantized in integer units (6.2). When we turn on $B^{(2)}$ background, the topological term becomes

$$
\begin{aligned}
Q & =\frac{1}{8 \pi^{2}} \int \operatorname{tr}\left[\left(\widetilde{F}-B^{(2)}\right) \wedge\left(\widetilde{F}-B^{(2)}\right)\right] \\
& =\underbrace{\frac{1}{8 \pi^{2}} \int \operatorname{tr}[(\widetilde{F} \wedge \widetilde{F})}_{\in \mathbb{Z}}-\underbrace{\left.\left.\frac{N}{8 \pi^{2}} \int B^{(2)} \wedge B^{(2)}\right)\right]}_{\in \frac{1}{N} \mathbb{Z}} \in \frac{1}{N} \mathbb{Z} .
\end{aligned}
$$

fractionally quantized, just like our simple quantum mechanics and $\mathbb{C P}^{N-1}$ examples. In the second line, the first term is integer quantized because $\widetilde{F}$ is in the $\mathrm{U}(N)$ bundle. The second term corresponds to $\mathbb{Z}_{N}$ 't Hooft flux background.

Let us now determine the saddles in the theory with non-trivial $B^{(2)}$ background, and their actions. The action functional for $\theta=0$ can be manipulated by using Bogomolny factorization:

$$
\begin{aligned}
S= & \frac{1}{g_{\mathrm{YM}}^{2}} \int \operatorname{tr}\left[\left(\widetilde{F}-B^{(2)}\right) \wedge \star\left(\widetilde{F}-B^{(2)}\right)\right] \\
= & \frac{1}{2 g_{\mathrm{YM}}^{2}} \int \operatorname{tr}\left[\left(\left(\widetilde{F}-B^{(2)}\right) \pm \star\left(\widetilde{F}-B^{(2)}\right)\right) \wedge \star\left(\left(\widetilde{F}-B^{(2)}\right) \pm \star\left(\widetilde{F}-B^{(2)}\right)\right)\right] \\
& \mp \frac{8 \pi^{2}}{g^{2}} \frac{1}{8 \pi^{2}} \int \operatorname{tr}\left[\left(\widetilde{F}-B^{(2)}\right) \wedge\left(\widetilde{F}-B^{(2)}\right)\right]
\end{aligned}
$$

The BPS bound is saturated by modified version of the instanton equation in the $B^{(2)}$ background:

$$
\left(\widetilde{F}-B^{(2)}\right)=\mp \star\left(\widetilde{F}-B^{(2)}\right)
$$

This equation is the counterpart of (2.29) in QM and (3.9) in $\mathbb{C P}^{N-1}$ in appropriate $\mathbb{Z}_{N}$ backgrounds. Using (6.12), we see that the action is bounded from below by

$$
S=\mp \frac{8 \pi^{2}}{g^{2}} \frac{1}{8 \pi^{2}} \int \operatorname{tr}\left[\left(\widetilde{F}-B^{(2)}\right) \wedge\left(\widetilde{F}-B^{(2)}\right)\right]=\frac{S_{I}}{N}
$$


When we turn on a $B^{(2)}$ flux, we are still in $\mathrm{SU}(N)$ theory, just like in our QM example, when we consider the $\operatorname{tr}\left[\mathrm{U}^{\ell} e^{-\beta H}\right]$, we are still in the $T_{N}$ model. If we sum over all backgrounds, we move to $\left(\mathrm{SU}(N) / \mathbb{Z}_{N}\right)_{p}$ gauge theory just like we moved to $\left(T_{N} / \mathbb{Z}_{N}\right)_{p}$ model in QM.

Formally, in quantum mechanics, it takes the insertion of $\mathrm{U}^{\ell}$ into the state sum to see explicitly the existence of fractional topological charge saddles. (Intuitively, just looking to the figures, this is of course obvious.) This enforces the following interpretation. We have to accept that in the $\mathrm{SU}(N)$ theory, saddles with fractional charge $\frac{1}{N} \mathbb{Z}$ and action $\frac{S_{I}}{N}=\frac{8 \pi^{2}}{g^{2} N}$ exist, but they are configuration in the $\mathrm{SU}(N) / \mathbb{Z}_{N}$ bundle. As such, all it takes is the insertion of a 't Hooft flux to see these configurations in the $\operatorname{PSU}(N)$ bundle in the original $\mathrm{SU}(N)$ theory. Then, we can build configurations in the $\mathrm{SU}(N)$ theory which live in the $\mathrm{SU}(N)$ bundle, which are non-BPS composites of objects in the $\operatorname{PSU}(N)$ bundle. Therefore, in exact analogy with our quantum mechanical example, there are topological configurations with fractional action, $\frac{2|n|}{N} S_{I}+|W| S_{I}$ and integer topological charge. This strongly suggests that the non-perturbative expansion parameter in the theory is $\mathrm{e}^{-S_{I} / N}=\exp \left[-\frac{8 \pi^{2}}{g^{2}(\mu) N}\right]$, which is exponentially more important than BPST instanton [9]:

$$
\mathrm{e}^{-\frac{S_{I}}{N}} \gg \mathrm{e}^{-2 \frac{S_{I}}{N}} \gg \mathrm{e}^{-3 \frac{S_{I}}{N}} \gg \ldots \gg \underbrace{\mathrm{e}^{-S_{I}}}_{\text {instanton }}
$$

Remarkably, the picture that emerges in the large 4-manifold $M_{4}$ is almost identical to our construction of dilute gas of monopole instantons on deformed YM and $\mathcal{N}=1 \mathrm{SYM}$ theory on $\mathbb{R}^{3} \times S^{1}$.

\section{1 $T^{3} \times S_{L}^{1}$ with 't Hooft flux and monopole-instantons}

We can use the idea of double-trace deformation both in $\mathrm{SU}(N)$ and $\mathrm{PSU}(N)$ theory, because the double-trace operator can be viewed as a trace in adjoint representation which belongs to both groups. Therefore, regardless of the existence of center symmetry, in both case, holonomy potential has a minimum at $\mathrm{e}^{\mathrm{i} \phi_{\star}}=\left(1, \omega, \omega^{2}, \ldots, \omega^{N-1}\right)$ at the center of Weyl chamber. Hence, in both case, dynamics abelianize to maximal abelian subgroup.

The magnetic monopoles that participate to non-perturbative dynamics have magnetic charges in root lattice, $\boldsymbol{\alpha} \in \Gamma_{r}$, both for $\mathrm{SU}(N)$ and $\operatorname{PSU}(N)$, corresponding to monopole operators $\mathcal{M}_{a}(x) \sim \mathrm{e}^{\mathrm{i} \boldsymbol{\alpha}_{a} \cdot \boldsymbol{\sigma}(x)}(5.4)$ in the effective field theory. The distinction between the two is that $\operatorname{PSU}(N)$ admits test magnetic charges $\boldsymbol{\mu} \in \Gamma_{w}$, which are forbidden in $\mathrm{SU}(N)$, corresponding to probe operators $\mathrm{e}^{\mathrm{i} \boldsymbol{\mu}_{a} \cdot \boldsymbol{\sigma}(x)}$ where $\boldsymbol{\mu}_{a}$ are fundamental weights. The magnetically charged operators which are non-trivial under $\Gamma_{w} / \Gamma_{r}$ are immediately in $\operatorname{PSU}(N)$ bundle, but how about dynamical monopoles $\boldsymbol{\alpha} \in \Gamma_{r}$ which carry $Q=1 / N$ ? What is the precise role they play?

Reminder about monopoles. Consider a monopole with charge $\boldsymbol{\mu} \in \Gamma_{w}$. Its magnetic field at asymptotic large distances is:

$$
\mathrm{B}=\frac{\widehat{\boldsymbol{r}}}{r^{2}}(\boldsymbol{\mu} \cdot \boldsymbol{H})
$$


where $\boldsymbol{H}=\left(H^{1}, \ldots, H^{N-1}\right)$ denote the Cartan generators of $\mathrm{SU}(N)$ algebra. For $\boldsymbol{\mu}$ which is a weight of fundamental representation, $(\boldsymbol{\mu} \cdot \boldsymbol{H})=\frac{T}{2 N}$ up to permutations, where $T=$ $\operatorname{diag}(1, \ldots, 1,-(N-1))$. If $\boldsymbol{\mu}$ is in $N$-index representation or adjoint representation, $\left(\boldsymbol{\mu}_{N} \cdot \boldsymbol{H}\right) \sim$ $(\boldsymbol{\alpha} \cdot \boldsymbol{H}) \sim \frac{T}{2}$.

Now, consider a sphere $S^{2}$ surrounding the monopole. The non-abelian gauge connection on the north and south hemisphere patches, corresponding to the above magnetic flux, is given by:

$$
\begin{aligned}
A_{\phi}^{N} & =\frac{1}{r} \frac{T}{2 N} \frac{(1-\cos \theta)}{\sin \theta} \\
A_{\phi}^{S} & =\frac{1}{r} \frac{T}{2 N} \frac{(-1-\cos \theta)}{\sin \theta}
\end{aligned}
$$

The two gauge connections are same up to gauge transformation. On a small strip along the equator where the patches overlap, we have

$$
A_{\phi}^{N}-A_{\phi}^{S}=\frac{1}{r \sin \theta} \partial_{\phi} T(\phi)
$$

where

$$
T(\phi)=\frac{\phi}{N} T \quad \text { and } \mathrm{e}^{\mathrm{i} T(\phi+2 \pi)}=\mathrm{e}^{\mathrm{i} T(\phi)} \mathrm{e}^{-\mathrm{i} \frac{2 \pi}{N}}
$$

i.e. charge $\boldsymbol{\mu}$ configuration lives in $\operatorname{PSU}(n)$ bundle. Concerning charge $\boldsymbol{\alpha}$ monopole, which is dynamical in the theory, we have the transition matrix $\left(\mathrm{e}^{\mathrm{i} T(\phi)}\right)^{N}$, which is an element of $\operatorname{PSU}(N)$ bundle which can lift to $\mathrm{SU}(N)$ bundle. But still, its topological charge is $1 / N$. We discuss its importance below.

Monopole-instantons in the 't Hooft flux background. In this part, we would like to see the role of monopole-instantons in the non-trivial 't Hooft flux background which is in the $\operatorname{PSU}(N)$ bundle, and determine an analytic solution to (6.14) when the theory is compactified on large $T^{3}$ (serving as regularization of $\mathbb{R}^{3}$ ) times a small $S_{L}^{1}$. First of all, following our construction in quantum mechanics and $\mathbb{C P}^{N-1}$ model, we know that we can undo the background and substitute it with boundary conditions.

Then, we can check if our monopole-instantons are sensible solutions within $\operatorname{PSU}(N)$, fitting with the boundary conditions. We will come to the conclusion that our monopoleinstantons with topological charge $Q_{a}=1 / N$, action $S_{a}=S_{I} / N$ and magnetic charge $\boldsymbol{\alpha}_{a}$ have an interpretation as tunneling events between configurations in the $\operatorname{PSU}(N)$ bundle in the semi-classical domain of the theory.

We can think turning on $k$ unit of 't Hooft magnetic flux in 12-plane, (in 3-direction), in multiple ways. The simplest way is to start with twisted boundary conditions on $T^{2} \times \mathbb{R}^{2}$, and undo the twist in favor of background $B_{12}^{(2)}$ fields. Gray band in figure 6 is a depiction of such classical background. This flux background exists without any reference to magnetic monopoles. On each $T^{2}$ slice, we have

$$
\frac{N}{2 \pi} \int B_{12}^{(2)}=k \equiv \ell_{12} \bmod N
$$

flux. A useful way to think about it is as follows. Such 't Hooft flux lines can be viewed as the field line created between magnetic monopoles with charges $\boldsymbol{\mu}_{k}$ and $-\boldsymbol{\mu}_{k}$, associated 
with magnetic $N$-ality $k$ and $-k$. (But recall that such charges are not present in the $\mathrm{SU}(N)$ theory, neither as dynamical object nor as probes. Even if when we gauge the 1 -form center symmetry, and move to say $\operatorname{PSU}(N)_{0}$ theory which is locally equivalent to $\mathrm{SU}(N)$ theory, such monopoles are not present dynamically. They are only allowed as probe charges.) These magnetic flux lines carry a magnetic flux through the 12-surface as well, given by

$$
\int_{12} \boldsymbol{B}=\frac{2 \pi}{g} \boldsymbol{\mu}_{k}
$$

So, we have to think that these flux lines are present and upper and lower plane in figure 6 are identified despite the fact that charges are not present in the theory. ${ }^{21}$ Another useful way to think about the $\ell_{12}=k$ flux line is a non-dynamical center-vortex, see [138] about center-vortices. Indeed, a center-vortex passing through a fundamental Wilson loop in 12-plane contributes to $W(C)=\mathrm{e}^{\mathrm{i} \oint a}$ as $\mathrm{e}^{\mathrm{i} \int_{12} B}=\mathrm{e}^{\mathrm{i} \frac{2 \pi k}{N}}$. All these perspectives are useful once we think about tunneling events in flux backgrounds.

Now, we can give an interpretation of the tunneling events corresponding to dynamical monopole-instantons with charge $\boldsymbol{\alpha}_{a} \in \Gamma_{r}$, in the SU $(N)$ theory on $T^{2} \times S_{\beta}^{1} \times S_{L}^{1}$ with $\ell_{12}=1$ unit of magnetic 't Hooft flux background, see figure 6. First, note that the configurations in the $\ell_{12}=1$ can be attached different magnetic flux, corresponding to any representative of the fundamental (defining) representation:

$$
\boldsymbol{\nu}_{1} \equiv \boldsymbol{\mu}_{1}, \quad \boldsymbol{\nu}_{2} \equiv \boldsymbol{\mu}_{1}-\boldsymbol{\alpha}_{1}, \quad \boldsymbol{\nu}_{3} \equiv \boldsymbol{\mu}_{1}-\boldsymbol{\alpha}_{1}-\boldsymbol{\alpha}_{2}, \quad \ldots \quad \boldsymbol{\nu}_{N} \equiv \boldsymbol{\mu}_{1}-\sum_{a=1}^{N-1} \boldsymbol{\alpha}_{a},
$$

The magnetic flux passing through the $T^{2}$ for these configurations are:

$$
\boldsymbol{\Phi}=\int_{T^{2}} \boldsymbol{B}=\frac{2 \pi}{g} \boldsymbol{\nu}_{a}, \quad a=1, \ldots, N .
$$

Assume that the flux is uniform through the surface of torus $T^{2}{ }^{22}$ Then for these $\mathrm{N}$ configurations, the energy of the corresponding states are exactly degenerate even at finite volume. If $\boldsymbol{B}_{a}=\frac{1}{A} \frac{2 \pi}{g} \boldsymbol{\nu}_{a}$, their energies are

$$
E_{a}=\frac{1}{2} \int_{T^{2}} \boldsymbol{B}_{a}{ }^{2}=\frac{1}{2 A}\left(\frac{2 \pi}{g}\right)^{2} \boldsymbol{\nu}_{a}^{2}=\frac{1}{2 A}\left(\frac{2 \pi}{g}\right)^{2}\left(1-\frac{1}{N}\right), \quad a=1, \ldots, N .
$$

The tunneling between the flux configurations $\left|\boldsymbol{\nu}_{a}\right\rangle$ and $\left|\boldsymbol{\nu}_{a+1}\right\rangle$ changes magnetic flux by simple and affine rooot $\boldsymbol{\alpha}_{a}, a=1, \ldots, N$, but does not change 't Hooft flux, which is fixed, $\ell_{12}=1$.

$$
\begin{aligned}
\Delta \int_{T^{2}} \boldsymbol{B}=\frac{2 \pi}{g}\left(\boldsymbol{\nu}_{a}-\boldsymbol{\nu}_{a+1}\right) & =-\frac{2 \pi}{g} \boldsymbol{\alpha}_{a}, \quad a=1, \ldots, N . \\
\Delta\left(\frac{N}{2 \pi} \int B_{12}^{(2)}\right) & =0
\end{aligned}
$$

\footnotetext{
${ }^{21}$ In certain sense, we can think of the 't Hooft magnetic flux $\ell_{12}=k$ sourced by $\boldsymbol{\mu}_{k}$ and $-\boldsymbol{\mu}_{k}$ pair similar to the mythical symbol ouroboros, a snake swallowing its own tail. We can forget about source and sink, and just take the flux lines into consideration.

${ }^{22}$ One does not need to make this assumption, but it makes the discussion a bit simpler, without sacrificing correctness. The 't Hooft flux insertion cost an energy, which depends only on $\ell_{12}=k$. For example, for $\ell_{12}=1$, it does not matter what magnetic flux out of the list (6.23) is associated with it.
} 


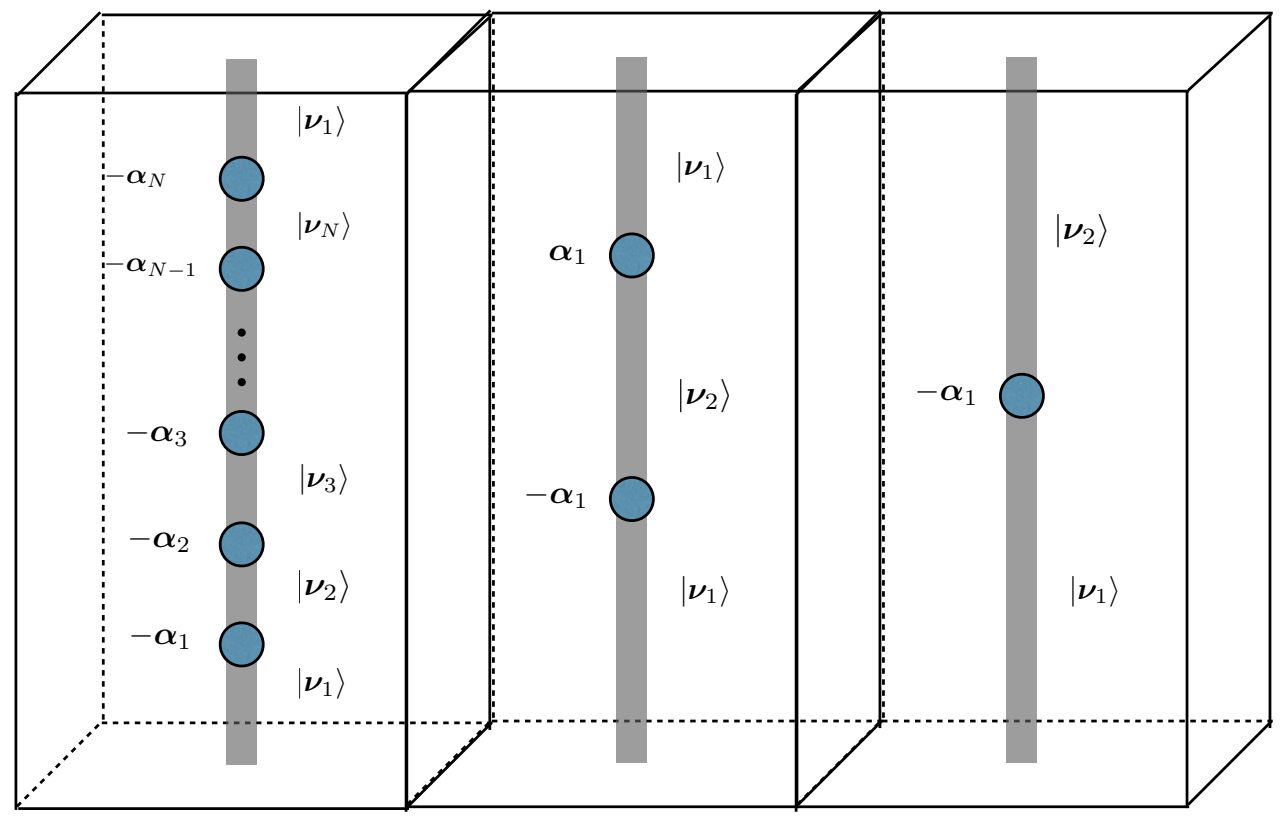

a) b) c)

Figure 6. Consider YM in the background of $\frac{N}{2 \pi} \int B_{12}^{(2)}=\ell_{12}=1$ unit of 't Hooft flux, $Z_{\ell_{12}}=$ $\operatorname{tr}\left[\mathrm{e}^{-\beta H_{\ell_{12}}}\right]$. The existence of $\ell_{12}=1$, in finite volume, leads to $N$-perturbatively degenerate minima, just like $T_{N}$ QM model. a) A non-trivial periodic (in Euclidean time) configuration with topological charge $W=-1$ contributing to $Z_{\ell_{12}}$ is a collection of $N$ dynamical monopole instantons, each of which have zero 't Hooft charge, but non-zero magnetic charge. b) A periodic configuration with topological charge $W=0$ contributing to $Z_{\ell_{12}}$. c) This configuration does not contribute to $Z_{\ell_{12}}$ as it is not periodic in Euclidean time, but it contributes to the sum over transition amplitudes,

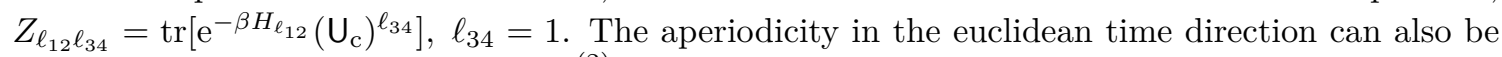
undone, and replaced with an $\frac{N}{2 \pi} \int B_{34}^{(2)}=\ell_{34}=1$ background.

This change in the magnetic flux is associated with the magnetic charge of the dynamical monopole instantons present in the theory.

\subsection{Two types of monopole events, Born-Oppenheimer and $T_{N}$ QM}

One needs to be careful concerning two distinct class of tunneling events in compactified set-up on $T^{2} \times S_{L}^{1} \times S^{1}$. These are between

a) Monopole tunneling events between exactly degenerate minima as described above, and

b) Tunnelings that become degenerate only in Area $\left(T^{2}\right) \rightarrow \infty$ limit.

The discussion above implies the existence of is different from the discussion of monopoleinstantons in the absence of 't Hooft flux background, for example, in the Polyakov model, see page 226 of ref. [139] (which is one of the rare textbooks which discusses Polyakov model and Hamiltonian interpretation of the tunneling.) A monopole-instanton in the 
case of Polyakov model always changes the energy of vacuum state at finite Area $\left(T^{2}\right)$. If $\boldsymbol{\Phi}=\int_{T^{2}} \boldsymbol{B}=\frac{2 \pi}{g} \boldsymbol{\alpha}_{a} n_{a}$ is magnetic flux (no summation over $a$ ), then the change in energy at finite volume between the zero-magnetic flux state and $\mathbf{\Phi}$ flux state is:

$$
\Delta E=\int_{T^{2}} \frac{1}{2} \boldsymbol{B}^{2}=\frac{1}{2} \frac{\left(\frac{2 \pi}{g}\right)^{2} n_{a}^{2}}{\operatorname{Area}\left(T^{2}\right)}>0
$$

Therefore, at finite volume, these states are not degenerate. Only when the area tends to infinity, the cost of energy becomes vanishingly small.

$$
\lim _{\operatorname{Area}\left(T^{2}\right) \rightarrow \infty} \Delta E=0
$$

and these states become degenerate with the zero magnetic flux state. These are the perturbative vacua, which only emerge in the Area $\left(T^{2}\right) \rightarrow \infty$ limit. The tunneling events between them is the Hamiltonian interpretation of monopole instantons in Polyakov model.

In the presence of 't Hooft flux background, say $\ell_{12}=1$, there is a genuine $N$-fold vacuum degeneracy at finite $\operatorname{Area}\left(T^{2}\right)$ associated with monopoles in the affine root system. The classical degenerate vacua at finite volume are the states $\left|\boldsymbol{\nu}_{a}\right\rangle, a=1, \ldots, N$. These are the counterpart of our perturbative $|j\rangle, j=1, \ldots, N$ in our quantum mechanical $T_{N}$ model. Therefore, in some respect, there are two distinct class of tunneling events in the case of $\ell_{12}=1$ background. For example, starting with the state $\left|\boldsymbol{\nu}_{1}\right\rangle$, tunneling with $-\boldsymbol{\alpha}_{1}$ and $+\boldsymbol{\alpha}_{1}$ takes us to states $\left|\boldsymbol{\nu}_{1}-\boldsymbol{\alpha}_{1}\right\rangle=\left|\boldsymbol{\nu}_{2}\right\rangle$, and $\left|\boldsymbol{\nu}_{1}+\boldsymbol{\alpha}_{1}\right\rangle=\left|2 \boldsymbol{\nu}_{1}-\boldsymbol{\nu}_{2}\right\rangle$.

$$
\begin{aligned}
& E_{\left|\boldsymbol{\nu}_{1}-\boldsymbol{\alpha}_{1}\right\rangle}-E_{\left|\boldsymbol{\nu}_{1}\right\rangle}=0 \\
& E_{\left|\boldsymbol{\nu}_{1}+\boldsymbol{\alpha}_{1}\right\rangle}-E_{\left|\boldsymbol{\nu}_{1}\right\rangle}=\frac{1}{2} \frac{\left(\frac{2 \pi}{g}\right)^{2} 4}{\operatorname{Area}\left(T^{2}\right)}>0 \underbrace{\longrightarrow}_{\text {Area }\left(T^{2}\right) \rightarrow \infty} 0
\end{aligned}
$$

where the first type is associated with the exact $N$-fold perturbative degeneracy at finite volume and the latter is associated with the emergent degeneracy as in the Polyakov model.

Since all magnetic flux states except for $\left|\boldsymbol{\nu}_{a}\right\rangle, a=1, \ldots, N$ have high energy in the small $T^{2} \times \mathbb{R} \times S_{L}^{1}$ limit, within Born-Oppenheimer approximation, the Yang-Mills theory with $\ell_{12}=1$ flux actually reduces to our simple $T_{N}$ model. The role of the fractional instantons $\mathcal{I}_{a}$ in the QM system is played by monopole-instantons with charge $-\boldsymbol{\alpha}_{a}$ in the Yang-Mills quantum mechanics. This is a concrete and nice correspondence with quantum mechanics and Yang-Mills theory. For example, it allows us to derive the vacuum energy densities for the $N$-branched vacua (5.16) and fractional theta angle dependence by simple quantum mechanics. However, we are more interested in the large- $T^{4}$ limit in general.

\subsection{What does $\mathbb{Z}_{N}$ TQFT background has anything to do with monopoles?}

Now, we can consider the partition function just in the background of 't Hooft flux $\ell_{12}$.

$$
Z_{\ell_{12}}=\operatorname{tr}\left[\mathrm{e}^{-\beta H_{\ell_{12}}}\right]=\int_{\mathrm{pbc}} \mathcal{D} a \mathrm{e}^{-S\left(a, B_{12}^{(2)}\right)}
$$


This is the exact counter-part of the regular partition function in our $T_{N}$ model with $N$ degenerate minima. The existence of potential in the QM model is a substitute for the effect of $\ell_{12}$ flux in Yang-Mills theory. To determine (6.30), we have to sum over all periodic configurations, in particular,

$$
\Phi(\beta)=\Phi(0)
$$

Therefore, exactly the same constraint that we obtained in the zero flux sector (5.10) is still operative, $n_{a}-\bar{n}_{a}=W, a=1, \ldots, N$ and independent of $a$ for dynamical monopoles of type $\boldsymbol{\alpha}_{a}$. The configurations contributing to the sum must satisfy magnetic neutrality, which automatically impose quantization of the topological charge $W \in \mathbb{Z}$.

Exactly as in our QM example, where in order to see what contributes to the state sum, we need to look to a transition matrix element, between the states $\left|\boldsymbol{\nu}_{a}\right\rangle$ and $\left|\boldsymbol{\nu}_{a+1}\right\rangle$. This is what is called an electric 't Hooft flux insertion. Let us make this more explicit.

The $\mathbb{Z}_{N}^{[1]}$ form center symmetry of the theory on $\mathbb{R}^{4}$, upon compactification on $T^{4} \equiv$ $T^{2} \times S_{L}^{1} \times S_{\beta}^{1}$, becomes $\left(\mathbb{Z}_{N}^{[0]}\right)^{4}$. The states $\left|\boldsymbol{\nu}_{a}\right\rangle$ described above are related to each other by a center-transformation, $\mathrm{U}_{\mathrm{c}}\left|\boldsymbol{\nu}_{a}\right\rangle=\left|\boldsymbol{\nu}_{a+1}\right\rangle$. Insertion of this operator in partition function corresponds to $\ell_{34}=1$ units of electric 't Hooft flux.

$$
\begin{aligned}
Z_{\ell_{12} \ell_{34}} & =\operatorname{tr}\left[\mathrm{e}^{\left.-\beta H_{\ell_{12}}\left(\mathrm{U}_{\mathrm{c}}\right)^{\ell_{34}}\right]}\right. \\
& =\sum_{a=1}^{N}\left\langle\boldsymbol{\nu}_{a}\left|\mathrm{e}^{-\beta H_{\ell_{12}}}\left(\mathrm{U}_{\mathrm{c}}\right)^{\ell_{34}}\right| \boldsymbol{\nu}_{a}\right\rangle \\
& =\sum_{a=1}^{N}\left\langle\boldsymbol{\nu}_{a}\left|\mathrm{e}^{-\beta H_{\ell_{12}}}\right| \boldsymbol{\nu}_{a+\ell_{34}}\right\rangle \\
& =\int_{\Phi(\beta)=\Phi(0)+\frac{2 \pi}{\mathrm{g}} \boldsymbol{\alpha}_{\mathrm{a}, \mathrm{a}+\ell_{34}}} \mathcal{D} a \mathrm{e}^{-S\left(a, B_{12}^{(2)}\right)} \\
& =\int_{\mathrm{pbc}} \mathcal{D} a \mathrm{e}^{-S\left(a, B_{12}^{(2)}, B_{34}^{(2)}\right)}
\end{aligned}
$$

In the fourth step, we have the boundary conditions corresponding to a transition amplitude:

$$
\Phi(\beta)=\Phi(0)+\frac{2 \pi}{g} \boldsymbol{\alpha}_{a, a+\ell_{34}} \equiv \Phi(0)+\frac{2 \pi}{g}\left(\boldsymbol{\nu}_{a}-\boldsymbol{\nu}_{a+\ell_{34}}\right)
$$

which is the change in the magnetic flux, corresponding to a dynamically allowed monopole event in the root lattice $\Gamma_{r}$. This is the twisted boundary condition in terms of magnetic flux description. In the last line of (6.32), we undo the twisted boundary condition into a periodic boundary condition, just like in the simple QM example, and transmute the effect to the background $B_{34}^{(2)}$ flux. Note that we can also think twisted boundary condition in different ways. In particular, we could have written it more conventionally as $U_{3}\left(\beta=L_{4}\right)=$ $\mathrm{e}^{\mathrm{i} \frac{2 \pi}{N} \ell_{34}} U_{3}(0)$. This is equivalent to above. This is because the center transformation acts on the center-symmetric holonomy field $\mathrm{e}^{\mathrm{i} \phi_{\star}}=\left(1, \omega, \omega^{2}, \ldots, \omega^{N-1}\right)$ as $\mathrm{e}^{\mathrm{i} \phi_{\star}} \rightarrow \mathrm{e}^{\mathrm{i} \frac{2 \pi}{N}} \mathrm{e}^{\mathrm{i} \phi_{\star}}$. The action is invariant under this transformation if we act on the fields by appropriate cyclic 
permutation, e.g, it takes $\sigma_{k} \rightarrow \sigma_{k-1}$ [140]. This cyclically permutes background magnetic flux, and corresponds to the twisted boundary condition on fourth line. ${ }^{23}$

The oddity of this result. On $\mathbb{R}^{3} \times S^{1}$, the action and topological charge of the monopole-instantons with magnetic charge $Q_{m}=\frac{2 \pi}{g} \boldsymbol{\alpha}_{a}$ is determined by center-symmetric gauge holonomy:

$$
\operatorname{Diag}\left(U_{3}\right)=\mathrm{e}^{\mathrm{i} \phi_{\star}}=\left(1, \omega, \omega^{2}, \ldots, \omega^{N-1}\right)
$$

This background is quantum mechanically stable in $\mathrm{QCD}(\mathrm{adj})$ and can be made stable in Yang-Mills by using the double trace deformation. Minimal action and topological charge in this background is given by

$$
S_{a}=\frac{4 \pi}{g^{2}}\left(\boldsymbol{\alpha}_{a} \cdot \boldsymbol{\phi}_{\star}\right)=\frac{8 \pi^{2}}{g^{2} N}, \quad Q=\frac{1}{2 \pi}\left(\boldsymbol{\alpha}_{a} \cdot \boldsymbol{\phi}_{\star}\right)=\frac{1}{N}
$$

On the other hand, it is also well-known that in the non-vanishing 't Hooft flux background, or equivalently, by coupling YM to $\mathbb{Z}_{N}$ TQFT, minimal action and topological charge are also $\frac{1}{N}$ quantized.

$$
S=\frac{8 \pi^{2}}{g^{2}} \frac{1}{8 \pi^{2}} \int B^{(2)} \wedge B^{(2)}=\frac{8 \pi^{2}}{g^{2} N}, \quad Q=\frac{N}{8 \pi^{2}} \int B^{(2)} \wedge B^{(2)}=\frac{\ell_{12} \ell_{34}}{N}=\frac{1}{N}
$$

Clearly, except for the results, these two constructions do not look anything alike. One is the action of a dynamical monopole instanton on the zero 't Hooft flux background and the other seems to be a property of the classical $B^{(2)}$ background associated with 1-form $\mathbb{Z}_{N}^{[1]}$ center symmetry. In the latter, there seems to be naively no data of the monopoleinstanton, and it is quite tempting that, despite the agreement of the final results, these two construction has nothing to do with one another.

But again, truth is subtler. Let us recap what we did in this section again because it is interesting. On $T^{2} \times S_{L}^{1} \times S_{\beta}^{1}$, assume first that center-symmetric background (6.35) is stabilized. Turning on $\ell_{12}=1$ on $T^{2}$ part gives us $N$ - exactly degenerate minima at the classical level. There are $N$-configurations which carry 1-unit of 't Hooft flux, but they also carry an orientation, that we labeled by $\boldsymbol{\nu}_{a}, a=1,2, \ldots$ in $(6.23) . \quad \ell_{34}=1$ background is equivalent to a twisted boundary condition $U_{3}\left(\beta=L_{4}\right)=\mathrm{e}^{\mathrm{i} \frac{2 \pi}{N} \ell_{34}} U_{3}(0)$. But as we explained above, this amounts to the transition amplitude between $\boldsymbol{\nu}_{a}$ and $\boldsymbol{\nu}_{a+1}$, meaning that the difference of the magnetic flux on the $\tau=\beta$ and $\tau=0$ is just the

${ }^{23}$ The gauge invariant definition of the $\mathrm{U}(1)^{N}$ photons are

$$
F_{\mu \nu, k}=\frac{1}{N} \sum_{p=0}^{N-1} \mathrm{e}^{-\mathrm{i} \frac{2 \pi k p}{N}} \operatorname{tr}\left(U_{3}^{p} F_{\mu \nu}\right)
$$

where $F_{\mu \nu}$ inside trace is the non-abelian gauge field strength. Crucially, counter-part of the adjoint Higgs field is the Polyakov loop. Under a zero-form center transformation, Polyakov loop transforms as $U_{3}^{p} \rightarrow \mathrm{e}^{\mathrm{i} \frac{2 \pi p}{N}} U_{3}^{p}$. Hence, the photon in the $3 \mathrm{~d}$ theory, transforms under the center transformation as $F_{\mu \nu, k} \rightarrow$ $F_{\mu \nu, k-1}$. The same is also true for the dual photon. Clearly, the monopole operator $\mathrm{e}^{\mathrm{i} \boldsymbol{\alpha}_{k} \cdot \boldsymbol{\sigma}} \rightarrow \mathrm{e}^{\mathrm{i} \boldsymbol{\alpha}_{k-1} \cdot \boldsymbol{\sigma}}$ and the flux states are cyclically shifted, $\left|\boldsymbol{\nu}_{k}\right\rangle \rightarrow\left|\boldsymbol{\nu}_{k-1}\right\rangle$. In other words, the zero-form part of center-symmetry acts like $\mathbb{Z}_{N}$ discrete translation symmetry in the $T_{N}$ model. 

charge $Q_{m}=\Delta \int_{T^{2}} \boldsymbol{B}=\frac{2 \pi}{g}\left(\boldsymbol{\nu}_{a}-\boldsymbol{\nu}_{a+1}\right)=-\frac{2 \pi}{g} \boldsymbol{\alpha}_{a}$. This is the charge of our dynamical
monopole-instanton event. ${ }^{24}$

Now, we realized our promise. To find the relevant saddles of $\mathrm{SU}(N)$ gauge theories, we should first consider either $\mathrm{SU}(N)$ with background gauge field $\left(B^{(2)}, B^{(1)}\right)$ for the $\mathbb{Z}_{N}^{[1]}$ 1-form symmetry or $\left(\mathrm{SU}(N) / \mathbb{Z}_{N}\right)_{p}$ theory where $\mathbb{Z}_{N}^{[1]}$ is gauged. After finding the configurations in the $\left(\mathrm{SU}(N) / \mathbb{Z}_{N}\right)_{p}$, we can patch them up to find the ones that can be lifted to $\mathrm{SU}(N)$ theory. These are fractional action (e.g. $2 S_{I} / N$ ) configurations that contribute to the partition function of $\mathrm{SU}(N)$ theory. Indeed, the mini-space formalism that we derived in section 5.1 reveals this fact. The construction and effective field theory based on monopole-instantons and bions works in semi-classical weak coupling domain. The fact that non-perturbative effects are controlled by action $\frac{S_{I}}{N}$ and topological charge $W=1 / N$ defects is true both in strong coupling domain and weak coupling domain.

We also note that $\mathbb{C P}^{N-1}$ model with an $\Omega_{F}$ and $\ell_{12}$ flux background and YangMills theory on the center-symmetric background with 't Hooft fluxes $\ell_{12}, \ell_{34}$ maps to identical problems from the point of view of mixed anomalies [141, 142]. For a general discussion of background field coupling to detect 't Hooft anomalies of 4d Yang-Mills, and their connection to $2 \mathrm{~d} \mathbb{C P}^{N ? 1}$, see also [143]. It is not a coincidence that the combination of (6.36) and (6.37) in $d=4$ is same as (3.37) in $d=2$.

Monopole-instantons in $\boldsymbol{B}^{(2)}$ background. In order to determine the fractional instantons on arbitrary $M_{4}$, we are supposed to solve in $4 \mathrm{~d}$ the BPS equation (6.14) in the $B^{(2)}$ background. If one of the directions is compactified, in one-lower dimension, the background field decomposes as $B_{4 \mathrm{~d}}^{(2)}=B_{3 \mathrm{~d}}^{(2)}+B_{3 \mathrm{~d}}^{(1)} \wedge \frac{\mathrm{d} x_{3}}{L_{3}}$ and the monopole instanton equations become

$$
\left(\widetilde{F}-B_{3 \mathrm{~d}}^{(2)}\right)=\mp \star\left(\widetilde{\mathrm{d}_{a} a_{3}}-B_{3 \mathrm{~d}}^{(1)}\right)
$$

However, as we did in quantum mechanics (compare (2.29) with (2.30)) and $\mathbb{C} P^{N-1}$ model, we can undo the background and convert it to boundary conditions that the monopole instanton has to satisfy. This is nothing but the regular monopole instanton equations $[144,145]$

$$
F=\mp \star \mathrm{d}_{a} a_{3}
$$

Our construction tells us that these well-known solutions fits with the 't Hooft flux boundary conditions and are indeed non-trivial solutions in $\operatorname{PSU}(N)$ bundle.

Remarks. Although we phrased the fact that monopole instantons with topological charge $Q=1 / N$ and action $\frac{8 \pi^{2}}{g^{2} N}$ fits perfectly with the $\mathrm{SU}(N)$ with twisted boundary conditions or $\operatorname{PSU}(N)$ bundle, the exact solutions within $\operatorname{PSU}(N)$ bundle had historically been a difficult endeavor. In 1981, 't Hooft showed that some constant abelian solutions that had topological charge

\footnotetext{
${ }^{24}$ If the minima for $U_{3}$ is classically center-broken configurations $U_{3}=\mathrm{e}^{\mathrm{i} \frac{2 \pi}{N} j} \mathbb{1}$ given in (A.5), then, the twisted boundary conditions $U_{3}\left(\beta=L_{4}\right)=\mathrm{e}^{\mathrm{i} \frac{2 \pi}{N} \ell_{34}} U_{3}(0)$ takes $|j\rangle$ to $\left|j+\ell_{34}\right\rangle$. This gives a dynamics which is different from above. See appendix A. It would be interesting to understand the connection between these two constructions more precisely.
} 
$1 / N$ and were easy to find, but had total action which never descends below $8 \pi^{2}(N-1) /\left(g^{2} N\right)$. Then, he found some non-abelian constant solutions that has action $8 \pi^{2} /\left(g^{2} N\right)$, a desired property. Historically, however, it was not easy to determine the time dependent or space-time dependent solutions. Gonzalez-Arroyo et al. found by simulations on latticized $T^{3} \times \mathbb{R}$ that time-dependent fractional instanton solutions with action $1 / N$ exist in the presence of 't Hooft flux [30, 31, 36, 37], see also [38] for recent work. One of our aim in this work was to determine these solutions analytically, and we were able to interpret the well-known monopole-instanton solutions on $\mathbb{R}^{3} \times S_{L}^{1}$ as non-trivial solutions in the flux background.

\subsection{Fractionalization in $\mathcal{N}=1 \mathrm{SU}(N)$ theory on $M_{4}$}

The evidence we gather suggest that the fundamentally important configurations in $\mathrm{SU}(N)$ gauge theory are fractional topological charge and fractional action instantons. The fact that such configurations exist is a mathematical statement about classification of the classical configurations, determined by the properties by the $\mathrm{SU}(N) / \mathbb{Z}_{N}$ bundle. However, one may ask, why such a statement, is not in apparent contradiction with the global chiral symmetry, ABJ anomaly or quantization of topological charge in $\mathrm{SU}(N)$ theories on orientable 4-manifolds $M_{4}$ ?

Let us consider $\mathcal{N}=1$ supersymmetric Yang-Mills theory with $G=\mathrm{SU}(N)$ gauge group. This model has a $\mathbb{Z}_{2 N}$ chiral symmetry which is broken dynamically to $\mathbb{Z}_{2}$ by the formation of fermion-bilinear condensate.

$$
\langle k|\operatorname{tr} \lambda \lambda| k\rangle=N \Lambda^{3} \mathrm{e}^{\mathrm{i} \frac{2 \pi k+\theta}{N}}, \quad k=0,1, \ldots, N-1
$$

leading to $N$ isolated supersymmetric vacua [29]. However, we claim that the classical configurations in this theory should be determined by using $\mathrm{SU}(N) / \mathbb{Z}_{N}$ bundle. But in a non-trivial flux background (6.11), the chiral symmetry is reduced to $\mathbb{Z}_{2}$, instead of $\mathbb{Z}_{2 N}$. Each of these configurations has two exact fermion zero modes. One may ask why the existence of objects with just 2 fermion zero modes is not in contradiction with the ABJ anomaly [146] in the $\mathrm{SU}(N)$ theory (with no background turned on). In particular, we expect

$$
\mathcal{I}_{a} \sim \mathrm{e}^{-\frac{S_{I}}{N}} \operatorname{tr} \lambda \lambda
$$

to be present in the Euclidean vacuum, even when the description of the vacuum is nonsemiclassical. Of course, the answer is tied with the fact that only integer topological charge configurations are in the $\mathrm{SU}(N)$ bundle, hence, only such configurations are part of the $\mathrm{SU}(N)$ theory. However, the key point is we can construct $\mathrm{SU}(N)$ configurations out of $\operatorname{PSU}(N)$ bundle in different ways. For example,

$$
\begin{aligned}
& I_{W=0}=\mathcal{I}_{a}\left(x_{a}\right) \overline{\mathcal{I}}_{a}\left(\bar{x}_{a}\right) \\
& I_{W=1}=\prod_{a=1}^{N} \mathcal{I}_{a}\left(x_{a}\right) \sim \mathrm{e}^{-S_{I}+i \theta} \prod_{a=1}^{N}(\operatorname{tr} \lambda \lambda)\left(x_{a}\right)
\end{aligned}
$$

are both in the $\mathrm{SU}(N)$ bundle, but not $\mathcal{I}_{a}\left(x_{a}\right)$ itself. As long as one fractional instanton here (at this spacetime point) has a friend fractional anti-instanton on the dark side of the 
Moon, the combination lives happily in the $\mathrm{SU}(N)$ bundle. The right hand side in (6.42) is the first configuration to reduce the chiral symmetry down to $\mathbb{Z}_{2 N}$ in $\mathrm{SU}(N)$ gauge theory. The proliferation of fractional instantons with the global constraint (5.10) is also capable of breaking the discrete chiral symmetry $[12,131]$ at weak coupling.

To see the presence of the fractional events on $\mathbb{R}^{4}$, let us reverse engineer the fractional instanton sum. Consider a soft mass deformation, $\Delta \mathcal{L}_{m}=\frac{m}{g^{2}} \operatorname{tr} \lambda \lambda+$ h.c. of $\mathcal{N}=1$ SYM. In this case, the vacuum energy density is modified into $\mathcal{E}_{k}=-\frac{m}{g^{2}}\langle k|\operatorname{tr} \lambda \lambda| k\rangle+$ c.c. at leading order in $m$. The partition function on a four manifold can be written as:

$$
Z(\theta)=\sum_{k=0}^{N-1} \mathrm{e}^{2 m N^{2} \Lambda^{3} V_{4} \cos \frac{\theta+2 \pi k}{N}}
$$

where $\Lambda^{3}=\mu^{3} \mathrm{e}^{-S_{I} / N}=\mu^{3} \mathrm{e}^{-\frac{8 \pi^{2}}{g^{2}(\mu) N}}$ is the strong scale and $V_{4}$ is the volume of the 4manifold that the theory is defined. Based on our quantum mechanical example, we can rewrite the partition function as

$$
\begin{aligned}
Z(\theta) & =N \sum_{W \in \mathbb{Z}} \sum_{n=0}^{\infty} \sum_{\bar{n}=0}^{\infty} \frac{1}{n !} \frac{1}{\bar{n} !}\left(m N^{2} \mu^{3} V_{4} \mathrm{e}^{-\frac{S_{I}}{N}+\mathrm{i} \frac{\theta}{N}}\right)^{n}\left(m N^{2} \mu^{3} V_{4} \mathrm{e}^{-\frac{S_{I}}{N}-\mathrm{i} \frac{\theta}{N}}\right)^{\bar{n}} \delta_{n-\bar{n}-W N, 0} \\
& =N \sum_{W \in \mathbb{Z}}\left[I_{N W}\left(2 m N^{2} \mu^{3} V_{4} \mathrm{e}^{-\frac{S_{I}}{N}}\right)\right] \mathrm{e}^{\mathrm{i} W \theta} \quad \text { (strong coupling, no 't Hooft flux) }
\end{aligned}
$$

A few remarks are in order:

- Individual terms in the sum are sourced by the solution of self-duality equation (6.14) in the $\mathrm{SU}(N) / \mathbb{Z}_{N}$ bundle. Minimal configurations have action $S_{I} / N$ and topological charge $\frac{1}{N}$, and they do not contribute to the partition function, but they contribute to physical observables.

- The constraint $\delta_{n-\bar{n}-W N, 0}$ guarantees that the sum is over integer topological charge configurations $W \in \mathbb{Z}$ which belong to $\mathrm{SU}(N)$. This is ultimately the reason why the proliferation of fractional instantons with the global constraint (6.44) is in agreement with ABJ anomaly, and integer quantization of topological charge on 4-manifolds, and is also capable of breaking chiral symmetry dynamically.

- The solutions in $\operatorname{PSU}(N)$ bundle has 4 bosonic zero modes, which may perhaps interpreted as the position moduli. $V_{4}$ may be viewed as the volume of the bosonic moduli. $\mu$ us Pauli-Villars renormalization scale. It appears with the combination $\mu^{n_{b}-n_{f} / 2}=\mu^{3}$ where $n_{b}=4, n_{f}=2$ are the numbers of bosonic and fermionic zero modes. The moduli space of an instanton in $\mathrm{SU}(N)$ theory, which has $N$ constituents, can be parametrized in terms of $N$-position, $a_{i} \in \mathbb{R}^{4}, i=1, \ldots, N$.

\section{Prospects and comments}

We developed three parallel constructions, in $d=1,2,4 \mathrm{QM}$ and QFTs. The analogous quantities are listed in table 1. The classical $N$-fold degenerate minima in QM is a consequence of the potential $V(N q)$. In $\mathbb{C P}^{N-1}$ it is induced by turning on $\Omega_{F}$ background for a 


\begin{tabular}{|c|c|c|c|c|}
\hline Theory & $\begin{array}{c}N \text {-fold classical } \\
\text { degeneracy }\end{array}$ & $\begin{array}{c}\text { Partially twisted } \\
\text { partition function }\end{array}$ & $\begin{array}{c}\text { Twisted } \\
\text { partition function }\end{array}$ & $\begin{array}{c}\text { Minimal topological charge } \\
\text { in TQFT background }\end{array}$ \\
\hline$T_{N}$ & $V(N q)$ & $Z=\operatorname{tr}\left[\mathrm{e}^{-\beta H}\right]$ & $Z_{\ell}=\operatorname{tr}\left[\mathrm{e}^{-\beta H} \mathrm{U}^{\ell}\right]$ & $\frac{1}{2 \pi} \int A^{(1)}=\frac{1}{N}$ \\
\hline $\mathbb{C P}^{N-1}$ & $\Omega_{F}$-twist & $Z_{\Omega_{F}}=\operatorname{tr}\left[\mathrm{e}^{-\beta H_{\Omega_{F}}}\right]$ & $Z_{\Omega_{F}, \ell}=\operatorname{tr}\left[\mathrm{e}^{-\beta H_{\Omega_{F}}} \mathrm{U}^{\ell}\right]$ & $\frac{1}{2 \pi} \int B^{(2)}=\frac{1}{N}$ \\
\hline $\mathrm{SU}(N) \mathrm{YM}$ & $\ell_{12}$ flux & $Z_{\ell_{12}}=\operatorname{tr}\left[\mathrm{e}^{-\beta H_{\ell_{12}}}\right]$ & $Z_{\ell_{12} \ell_{34}}=\operatorname{tr}\left[\mathrm{e}^{-\beta H_{\ell_{12}}} \mathrm{U}^{\ell_{34}}\right]$ & $\frac{N}{8 \pi^{2}} \int B^{(2)} \wedge B^{(2)}=\frac{1}{N}$ \\
\hline
\end{tabular}

Table 1. The correspondence between the $T_{N}$ quantum mechanics, $\mathbb{C} P^{N-1}$ with flavor twisted background, and Yang-Mills with 't Hooft flux background.

global symmetry, and in the YM theory, it is induced by turning on $B^{(2)}=\ell_{12}$ background in the 12-plane. The simplest way to realize that these $N$-fold classical degeneracy is not a fiction is to recall that in $\mathbb{C P}^{N-1}$ and YM theory, the vacua at the quantum level is $N$-branched. The classical (as well as all orders perturbative minima) that emerges in the second column is the precursor of that well-know fact. In Yang-Mills, in the 3-direction associated with circle $S_{L}^{1}$, we may or may not have a center-symmetric holonomy field $U_{3}$. Our main application is in center-symmetric background, but the application in the appendix A is in classically center-broken background. The $N$-fold perturbative degeneracy is independent of that. The generalized partition function in third column is periodic in $\beta$ and it receives contribution only from $W \in \mathbb{Z}$ integer topological charge configurations. The important point is that these configurations can and do have fractional action, which starts with $\frac{2 S_{I}}{N}$, in the $W=0$ sector. Observables are controlled by $\frac{S_{I}}{N}$. To see these configurations more explicitly, we inspect the fully twisted partition functions, also involving a twist associated with $S_{\beta}^{1}$ circle (say, the 't Hooft flux in 34-plane in gauge theory and 12 -plane in $\mathbb{C P}^{N-1}$ ). We are free to put all twists as boundary conditions. Alternatively, we can use periodic boundary conditions everywhere, and turn on classical background fields. This is equivalent to coupling the QFT to $\mathbb{Z}_{N} \mathrm{TQFT}$, and reveals the true nature of non-perturbative configurations in the original theory without any backgrounds.

Pure Yang-Mills theory on $\mathbb{R}^{4}$. Coupling YM theory to a $\mathbb{Z}_{N}$ TQFT tells us that we need to use $\operatorname{PSU}(N)$ bundle to build up $W \in \mathbb{Z}$ configurations in the $\mathrm{SU}(N)$, which generically possess fractional action. The construction instructs us that non-perturbative expansion parameter in pure $\mathrm{SU}(N)$ Yang-Mills theory is

$$
\mathrm{e}^{-\frac{S_{I}}{N}+\mathrm{i} \frac{\theta}{N}}=\mathrm{e}^{-\frac{8 \pi^{2}}{g^{2}(\mu) N}+\mathrm{i} \frac{\theta}{N}}
$$

This is exactly like the monopole-instanton factor in deformed Yang-Mills theory on $\mathbb{R}^{3} \times$ $S^{1}[14]$ where $\mu$ is Pauli-Villars scale. This parameter is exponentially more important than the $4 \mathrm{~d}$ instanton amplitude [9].

QCD and general SU $(N)$ gauge theory, NP expansion parameter. In his original works, 't Hooft argues that the twisted boundary conditions cannot be applied to QCD with fundamental quarks as there is no longer a center symmetry. Though the absence of center symmetry is a correct statement, his negative conclusion can be avoided in two different ways. 
- If $\operatorname{gcd}\left(N_{f}, N\right) \neq 1$, we can impose 't Hooft twisted boundary conditions by turning on first an $\mathrm{SU}\left(N_{f}\right)$ background. This becomes most efficient for $N_{f}=N$, where we can show that expansion parameter becomes $\exp \left[-S_{I} / N+\mathrm{i} \theta / N\right]$.

- If we turn on $\mathrm{U}(1)_{V} / \mathbb{Z}_{N}$ background [147] (which is part of genuine vector-like symmetry of the theory), then, we can impose twisted boundary conditions even for $N_{f}=1$. In fact, our construction in $\mathbb{C P}^{N-1}$ is the dual of this idea, where we had $\mathrm{U}(1)$ gauge structure, and $\mathrm{SU}(N) / \mathbb{Z}_{N}$ global symmetry, see section 3.1. In 1-flavor QCD, we have $\mathrm{SU}(N)$ gauge structure and $\mathrm{U}(1) / \mathbb{Z}_{N}$ global symmetry. In either case, we can impose the same twists. As a result, we were able to turn on 't Hooft flux in a theory with quarks. Therefore, the expansion parameter is always $\exp \left[-8 \pi^{2} / g^{2} N\right]$ regardless of matter content of $\mathrm{SU}(N)$ gauge theory.

Renormalization group for topological defects. If $\exp \left[-8 \pi^{2} / g^{2} N\right]$ is expansion parameter in all $\mathrm{SU}(N)$ QCD-like theories, what distinguishes an asymptotically free IR-CFT from a confining theory which exhibit chiral symmetry breaking and confinement? We need to formulate a renormalization group, similar to $[18,86]$, which tells us relevance vs. irrelevance of defect operators. Indeed, we used such a method at $\theta=\pi$ of $\mathbb{C P}^{1}$ model to exhibit the irrelevance of doubly-charged vertex operators and show conformality, and to prove the relevance of vertex operators at $\theta=0$, and mass gap.

Rethinking deformed Yang-Mills theory on $\mathbb{R}^{\mathbf{3}} \times \boldsymbol{S}^{\mathbf{1}}$. On $\mathbb{R}^{3} \times S^{1}$, a monopoleinstanton possesses four bosonic zero mode. This introduces the Pauli-Villars $\mu$ dependence of the form $\mu^{4}$. The fluctuation operator over the non-zero modes in the background of a monopole gives $\left[\operatorname{Det}\left(-D^{2}\right)\right]^{-1}=\left(\mu r_{\text {mon }}\right)^{-1 / 3}$ where $r_{\text {mon }}=m_{W}^{-1}=\frac{L N}{2 \pi}$ is the characteristic size of monopole-instantons, which is the inverse $W$-boson mass, determined by the adjoint Higgsing. The combination of the zero and non-zero modes just gives the expected result, $\mu^{4-(1 / 3)}=\mu^{11 / 3}$ which combines with the exponential to give the 1-loop renormalization group invariant scale,

$$
\mu^{11 / 3} \mathrm{e}^{-\frac{8 \pi^{2}}{g^{2}(\mu) N}+\mathrm{i} \frac{\theta}{N}}=\Lambda^{11 / 3} \mathrm{e}^{\mathrm{i} \frac{\theta}{N}}
$$

The mass gap on semi-classical domain on $\mathbb{R}^{3} \times S^{1}$ is given by:

$$
m^{2}(\theta)=\Lambda^{2}(\Lambda L N)^{5 / 3}\left[\operatorname{Max}_{k} \cos \left(\frac{\theta+2 \pi k}{N}\right)\right], \quad L N \Lambda \ll 1, \text { semiclassical domain }
$$

This result is based on a justified effective field theory based on proliferation of monopoleinstantons. The merit of this formula is that it captures the $\theta$ angle dependence and existence of mass gap correctly. As mentioned above, $L N$ is the monopole-instanton size $r_{\text {mon }}$, and semi-classics is reliable provided $r_{\text {mon }} \Lambda \lesssim 1$.

One of the new insights of this work is to recognize that the monopole-instantons can also be interpreted as tunneling events in the $\operatorname{PSU}(N)$ bundle, in a fixed 't Hooft flux sector. See figure 6. This may seem a trivial observation, but this interpretation is important, because 't Hooft himself, in 1981, was only capable of finding constant solutions on $T^{4}$ on $\operatorname{PSU}(N)$ bundle and these solutions required certain ratio of scales in $T^{4}$ geometry 
to achieve BPS bound. (He says in his paper, "Considering the difficulty we had in finding them it looked worth-while to publish the result." [27]). After this work, the only nontrivial solutions that has been obtained were via numerical lattice simulations. See [38] for an up to date report.

The fact that monopole-instantons are in $\operatorname{PSU}(N)$ bundle makes one wonder if they may be weak coupling reincarnation of configurations with action $S_{I} / N$ on arbitrarily large $T_{\text {large }}^{4} \sim \mathbb{R}^{4}$ down to $T_{\text {large }}^{3} \times S_{\text {small }}^{1} \sim \mathbb{R}^{3} \times S_{\text {small }}^{1}$. It is tempting to speculate that on $\mathbb{R}^{4}$, the fractional instantons can produce a gap of the form $m^{2}(\theta)=$ $\Lambda^{11 / 6}(\rho)^{5 / 6}\left[\operatorname{Max}_{k} f\left(\frac{\theta+2 \pi k}{N}\right)\right]^{p}+\ldots$ where $\rho$ is a size moduli of instanton, and $p$ can be a real number (not necessarily one as in semi-classical domain) of order one. (Recall that in $\mathbb{C P}^{1}$, we showed that mass gap is of the form $m(\theta)=\Lambda|\cos (\theta / 2)|^{2 / 3}$.) Very likely, similar to $\mathbb{C P}^{N-1}$ where Debye length provides a self-consistent cut-off over the instanton size moduli (4.58), there may be a similar mechanism on $\mathbb{R}^{4}$ where $\rho$ is cut-off at order $\Lambda^{-1}$.

k-strings and center-vortices. In pure Yang-Mills, there is only one fundamental string, and $k$-string tensions are determined by $N$-ality of representation of sources in the strong coupling domain. However, in Polyakov model on $\mathbb{R}^{3}[66]$ and Seiberg-Witten theory on $\mathbb{R}^{4}$ [148], there are $N-1$ types of fundamental strings, with $N-1$ different string tensions, astray from the pure Yang-Mills theory (but an unavoidable property of these theories, see e.g. [149]). In deformed YM, $\mathcal{N}=1 \mathrm{SYM}$ or $\mathrm{QCD}(\operatorname{adj})$ on $\mathbb{R}^{3} \times S^{1}$, there is again only one fundamental string tension, just like pure Yang-Mills, thanks to unbroken $\mathbb{Z}_{N}$ center symmetry and the absence of elementary adjoint Higgs field [150].

Despite this remarkable fact, it is important to notice that the infrared physics of deformed Yang-Mills theory (as well as Polyakov model [70]) cannot be described by just writing an EFT based on proliferation of monopole-instantons, one should keep in mind the existence of $W$-bosons as emphasized $[138,151]$. If one forgets $W$-bosons, this would lead to non-zero tension for adjoint or $k=N$ string, which is incorrect, as well as quantitatively incorrect string tensions for $k>N / 2$.

But fortunately, dynamical $W$-bosons are in the full microscopic theory, and they can easily screen $N$-ality zero sources. There is no doubt that:

i) In the weak coupling semiclassical regime on $\mathbb{R}^{3} \times S^{1}$ center-symmetric background, there exists an EFT description in terms monopole-instanton and bion local fields (ie, fields which create local excitations with the quantum numbers of the fractionalinstanton along with their fermionic zero modes, and local bion fields). A number of low-energy observables, mass gap, topological susceptibilities, chiral condensates, and $N$-ality $k \lesssim N / 2$ (single winding) Wilson loop expectation values are accurately (and sometimes exactly) described by the local EFT based on these topological defects in the semi-classical domain. This is as explicit as it can ever be [12, 14, 131, 152], and there is no doubt of its validity either in supersymmetric or non-supersymmetric theories.

ii) However, to screen the adjoint probes, and to get the right string tensions for $k \gtrsim$ $N / 2$, one must keep $W$-bosons in the description, (which we should not call EFT 
anymore), otherwise these observables will come out to be wrong. In other words, Wilson loops with $k \gtrsim N / 2$ is not in the set of low-enegy observable that EFT can address correctly.

iii) We do not know a semi-classical effective field theory describing the role of centervortices, even when a large subset of non-perturbative observables are completely describable in terms of semi-classics and EFT, as in $\mathcal{N}=1 \mathrm{SYM}$ or $\mathrm{QCD}$ (adj) on $\mathbb{R}^{3} \times S^{1}$, or Polyakov model on $\mathbb{R}^{3}$.

The EFT for deformed YM on small $\mathbb{R}^{3} \times S^{1}$ center-symmetric regime is based on the parametric separation of scales between the non-perturbative dual photon mass $m_{\gamma} \sim$ $\Lambda(\Lambda L N)^{5 / 6}$ and the $W$-boson mass, $\frac{2 \pi}{L N} \sim \Lambda(\Lambda L N)^{-1}$, and EFT is valid provided

$$
\frac{m_{\gamma}}{m_{W}} \sim(\Lambda L N)^{11 / 6} \lesssim 1
$$

But as we emphasized above, the EFT can be used to determine some observables, but not all observables. For example, it is not capable of producing $k \gtrsim N / 2$ string tensions or multi-winding loops for some $k \lesssim N / 2$ probes. To produce those correctly, we must incorporate into EFT $W$-bosons, the heavy degrees of freedom, which are not part of EFT. Once this is done, one cannot call the combined theory as EFT, better call it microscopic theory. But it has an EFT subsector.

Perhaps, a reasonable proposal is to aim to demonstrate that monopole-instanton + bions $+W$ bosons $\}$ system is equivalent to center-vortices in weak coupling domain. In some ways, it seems like one needs to incorporate $W$-bosons to the description and then, integrate it out. But then, very likely, there will not be a local description. In other words, we suspect that there may not be a local semi-classical EFT description for center-vortices at all.

But we still think that center vortices is a major part of the story, very likely not more or less important than fractional instantons, and the two are possibly intimately connected. There are some other interesting facts about the center-vortices that support this perspective. 't Hooft magnetic flux background itself is actually a (non-dynamical) center vortex, and dynamical monopole-instanton tunneling events can be viewed as junction on them (See figure 6) and do not change the charge of 't Hooft background as it has zero 't Hooft charge. On $T^{4}$, the intersection point of center-vortices acts as sourcing topological charge $W=1 / N$ [153], which naturally lives in $\operatorname{PSU}(N)$ bundle, and smoothed centervortex configurations are related to instantons [154], which we proved to be composites of fractional instantons with $W=1 / N$ even on large $T^{4}$ strong coupling domain.

Finally, probably most importantly, when we couple $\mathrm{SU}(N)$ Wilson lattice gauge theory [155] to $\mathbb{Z}_{N}$ TQFT, and gauge it, vortices become local minima of the action for any $N$, obviating various restrictions concerning stability of center-vortex configurations $[151,156]$. However, one should be careful with the fact that there are multiple lattice $\operatorname{PSU}(N)_{0}$ theories (with or without magnetic matter), and the one which is locally same as $\mathrm{SU}(N)$ theory should not have magnetic matter charged under magnetic center $N$-ality. We are currently investigating $\operatorname{PSU}(N)_{0}$ lattice gauge theories (with or without magnetic matter) in order to reveal these connections precisely. 
The importance of reparametrization of instanton moduli space. The instanton in $\mathrm{SU}(N)$ gauge theory on $\mathbb{R}^{4}$ has $4 N$ bosonic zero modes and $q$-instantons have $4 N q$ moduli parameters. The analogy with the analysis of refs. [16, 17] suggest that perhaps, we should parametrize the moduli space of instanton as

$$
\left\{a_{j}^{m}\right\}, \quad m=1, \ldots q, j=1, \ldots, N
$$

These are $N q 4$-positions (or quaternions) that we may think as fractional instanton positions.

An intriguing fact that we learned in the course of this work is that the monopoleinstantons on $\mathbb{R}^{3} \times S^{1}$ are in $\operatorname{PSU}(N)$ bundle and come exactly as $\mu^{11 / 3} \exp \left[-\frac{8 \pi^{2}}{g^{2} N}+\mathrm{i} \frac{\theta}{N}\right]$ as we already knew from [14].

- The non-perturbative expansion parameter in all center symmetric theories in weak coupling domain on $\mathbb{R}^{3} \times S^{1}$ is this factor.

- The non-perturbative expansion parameter in the strongly coupled domain on $\mathbb{R}^{4}$ for all theories that admit a coupling to $\mathbb{Z}_{N}$ TQFT is the same non-perturbative factor.

It is hard to believe that this is an accident.

The picture we obtain from $\mathbb{R}^{2}$ and $\mathbb{R} \times S^{1}$ suggests something deeper, compliant with the previous paragraph. The instanton solutions of $[16,17]$ on $\mathbb{R}^{2}$ do not physically break an instanton into pieces. (Action density and topological density are single lumps for one instanton). It is a reparametrization of the usual solution. The brilliant thing is that the fluctuation determinant in the background of parametrization (4.3) admits an interpretation as if we are summing over fractional vortex instantons, which carry fractional charge and fractional action, as it appears in (4.6). To see the fractional instantons explicitly, we need to do an extra work, for example, couple the theory to $\Omega_{F}$ background (see [10] for a review), or 't Hooft flux background, or consider it in non-trivial holonomy background [125-129], and then, dial the moduli parameters. For small moduli, one still does not see physical separation, and only for sufficiently large moduli parameters, one sees fractionalization explicitly (see figure 4). May be, BPST instanton is far more clever than us, and it hides its deepest secrets inside in its moduli parametrization.

QCD(F) on $\mathbb{R}^{4}$ vs. $\mathbb{R}^{\mathbf{3}} \times \boldsymbol{S}^{\mathbf{1}}$. Think of QCD with fundamental quarks, on $M_{4}$. Turn on a background for the global symmetry $\mathrm{U}(1)_{V} / \mathbb{Z}_{N}$, upon which we can couple the system to $\mathbb{Z}_{N}$ TQFT. Our claim is that this system should admit topological excitations that are controlled by the action $S_{I} / N$, and these are relevant to real QCD on $\mathbb{R}^{4}$.

Consider, for example, QCD with $N_{f}$ flavors on $\mathbb{R}^{3} \times S^{1}$. For concreteness, let us take $N_{f} \leq N$, but $N_{f}$ can be made arbitrary. Then, in a center-symmetric background for gauge holonomy, we can also turn on an $\Omega_{F} \in \mathrm{SU}\left(N_{f}\right)$ as in (3.29). Then, we have $N$ monopoles with action $S_{I} / N . N-N_{f}$ of the monopoles do not have a fermi zero mode (call this set $\mathcal{S}_{1}$ ) and $N_{f}$ of them have 2 fermi zero modes each (call this set $\mathcal{S}_{2}$ ). This is the set-up introduced in $[85,157]$ to explain the transmutation of $\frac{\theta+2 \pi k}{N_{f}}$ dependence in $N_{f}$ flavor QCD to the $\frac{\theta+2 \pi k}{N_{c}}$ in pure Yang-Mills as the fermions are decoupled by increasing their masses. 
Monopole instantons on $\mathcal{S}_{1}$ each has 4 bosonic zero modes, 3 position and one angular. Perhaps, in the light of our intentions, it is wise to denote $\mathrm{d}^{3} a \mathrm{~d} \phi \equiv L^{-1} \mathrm{~d}^{4} a$. Monopole instantons on $\mathcal{S}_{2}$ each has 4 bosonic zero modes, 3 position and one angular, and 2 fermi zero modes.

Then, the measure, in the background of the monopole instantons, which amounts to the calculation of pre-exponential factor, takes the form:

$$
\mathrm{d} \mu_{\text {mon }, j} \sim \begin{cases}\mu^{4} d^{4} a \mathrm{e}^{-\frac{8 \pi^{2}}{g^{2} N}+\mathrm{i} \frac{\theta}{N}} \underbrace{\left(\operatorname{det}^{\prime}\left[-D^{2}\right](\mu)\right)^{-1},}_{\left(r_{\text {mon }} \mu\right)^{-1 / 3}} & \\ =\mu^{4-\frac{1}{3}} \mathrm{e}^{-\frac{8 \pi^{2}}{g^{2} N}+\mathrm{i} \frac{\theta}{N}} d^{4} a \ldots & j \in \mathcal{S}_{1} \\ \mu^{4} d^{4} a \mu^{-1} d^{2} \xi_{f} \mathrm{e}^{-\frac{8 \pi^{2}}{g^{2} N}+\mathrm{i} \frac{\theta}{N}} \underbrace{\left(\operatorname{det}^{\prime}\left[-D^{2}\right](\mu)\right)^{-1}}_{\left(r_{\text {mon }} \mu\right)^{-1 / 3}} \underbrace{\left(\operatorname{det}_{\mathcal{R}}^{\prime}\left[\gamma_{\mu} D_{\mu}\right](\mu)\right)}_{\left(r_{\text {mon }} \mu\right)^{1 / 3}}, & j \in \mathcal{S}_{2} \\ =\mu^{4-\frac{1}{3}} \mu^{-1+\frac{1}{3}} \mathrm{e}^{-\frac{8 \pi^{2}}{g^{2} N}+\mathrm{i} \frac{\theta}{N}} d^{4} a d^{2} \xi_{f} \ldots & j\end{cases}
$$

where det ${ }^{\prime}$ is the fluctuation operator over non-zero modes, which also induces $\mu$-scale dependence.

$$
\begin{aligned}
\mathcal{I}_{\mathrm{BPST}} \sim \prod_{j \in \mathcal{S}_{1}} \mathcal{M}_{j} \prod_{j \in \mathcal{S}_{2}} \mathcal{M}_{j} & \sim\left(\mu^{4-\frac{1}{3}} \mathrm{e}^{-\frac{8 \pi^{2}}{g^{2} N}+\mathrm{i} \frac{\theta}{N}}\right)^{N-N_{f}}\left(\mu^{4-\frac{1}{3}} \mu^{-1+\frac{1}{3}} \mathrm{e}^{-\frac{8 \pi^{2}}{g^{2} N}+\mathrm{i} \frac{\theta}{N}}\right)^{N_{f}} \\
& =\mu^{\frac{11}{3} N-\frac{2}{3} N_{f}} \mathrm{e}^{-\frac{8 \pi^{2}}{g^{2}}+\mathrm{i} \theta}
\end{aligned}
$$

The part of this expression involving Pauli-Villars scales and how it combines to produce renormalization group invariant strong scale by combining $N$ constituent looks like an elegant conspiracy. The standard calculations of the pre-exponential factor for a BPST instanton (in the standard parametrization) can be found in NSVZ review [21].

It is very tempting that the structure above arises in $\mathbb{R}^{4}$ when $\mathrm{QCD}(\mathrm{F})$ is considered on $\mathrm{U}(1)_{V} / \mathbb{Z}_{N}$ background and the theory is coupled to a $\mathbb{Z}_{N}$ TQFT. We suspect that in the decompactification to $\mathbb{R}^{4},\left(r_{\text {mon }} \mu\right)$ type factors on $\mathbb{R}^{3} \times S^{1}$ will be replaced by combinations such as $\left(\left|a_{j}^{m}-a_{k}^{n}\right| \mu\right),\left(\left|\bar{a}_{j}^{m}-\bar{a}_{k}^{n}\right| \mu\right)$, or $\left(\left|a_{j}^{m}-\bar{a}_{k}^{n}\right| \mu\right)$. Once we consider original QCD on $\mathbb{R}^{4}$, we should probably inspect proliferation of the fractional instantons which should appear in a way similar to monopole-instantons do on $\mathbb{R}^{3} \times S^{1}$. They must obey the global constraints similar to monopole-instantons, and we should see if one can obtain a self-consistent construction of QCD vacuum for general $N_{f}$.

\section{Acknowledgments}

I am deeply grateful to Aleksey Cherman, Yuya Tanizaki, Tin Sulejmanpasic for many discussions at odd hours. I am also thankful to Margarita Garcia Perez, Thomas Schäfer, Patrick Draper, Mohamed Anber, Gerald Dunne, Tony Gonzalez-Arroyo, Mikhail Shifman, David Gross, Philip Argyres, Rob Pisarski, Pierre van Baal for past discussions. Although I have not met with Polyakov in person, I am thankful to him for his unpretentious beautiful book. My work is supported by the U.S. Department of Energy, Office of Science, Division of Nuclear Physics under Award DE-SC0013036. 


\section{A Yang-Mills with flux on $1^{3} \times S_{\beta}^{1}$ lattice vs. $T_{N}$ model}

Let us consider a $4 \mathrm{~d}$ lattice gauge theory with discrete space and continuous time, $N_{s}^{3} \times \mathbb{R}$. Then, to reduce the model to simple quantum mechanics, we take $N_{s}=1$ in the same spirit with the twisted Eguchi-Kawai model [45, 158, 159], by employing twisted boundary conditions, but we do not take the large- $N$ limit.

We consider 1-site theory with Lagrangian

$$
g^{2} \mathcal{L}=\operatorname{tr}\left|\mathrm{d} \widetilde{U}_{i}\right|^{2}+\operatorname{tr}\left|\left[\widetilde{U}_{i}, \widetilde{U}_{j}\right]\right|^{2}
$$

in the $A_{0}=0$ gauge. In path integral, we assume that $\widetilde{U}_{i}$ obey 't Hooft twisted boundary conditions on the $1^{3}$ lattice.

$$
\widetilde{U}_{i, \mathbf{n}+N_{j} \mathbf{e}_{\mathbf{j}}}=\Omega_{j} \widetilde{U}_{i, \mathbf{n}} \Omega_{j}^{\dagger}
$$

Consistency at, e.g. $N_{1} \mathbf{e}_{1}+N_{2} \mathbf{e}_{2}$ requires (recall $N_{1}=N_{2}=1$ ):

$$
\Omega_{1} \Omega_{2}=\Omega_{2} \Omega_{1} \mathrm{e}^{\mathrm{i} \frac{2 \pi}{N} \ell_{12}}
$$

where $\ell_{12}$ is $B^{(2)}$ flux $\bmod N$. Since lattice is only 1-site, this forces the transitions matrices to be independent of space coordinates. This actually leads to subtle and important differences between an arbitrarily small continuum $T^{3} \times S_{\beta}^{1}$ theory and $1^{3} \times S_{\beta}^{1}$ theory. In the former, the transition matrices are allowed to depend on spacetime, but not in the latter, and this has consequences.

Undoing the twisted boundary conditions in favor of a classical background field, we end up with the flux $\ell_{12}$ in the 12 face and the matrix model becomes

$$
g^{2} \mathcal{L}=\operatorname{tr}\left|\mathrm{d} U_{i}\right|^{2}+\operatorname{tr}\left|\left[U_{1} U_{2}-\mathrm{e}^{\mathrm{i} \frac{2 \pi}{N} \ell_{12}} U_{2} U_{1}\right]\right|^{2}+\operatorname{tr}\left|\left[U_{1}, U_{3}\right]\right|^{2}+\operatorname{tr}\left|\left[U_{2}, U_{3}\right]\right|^{2}
$$

where path integral over the $U_{i}(\tau)$ field is periodic both in time and space direction (which is reduced to 1 -site).

Let us describe the classical minima of this system. The minimum of the modified commutator (take $\ell_{12}=1$ ) is $U_{1}=C, U_{2}=S$, where $C$ and $S$ are clock and shift matrices. The minima of the action correspond to the values of $U_{3}$ which commutes both with $C$ and $S$. Since $C$ and $S$ are generators of $\mathrm{GL}(N, \mathbb{C})$ algebra, the only matrix that commutes with both is proportional to identity. Since $U_{3}$ lives in $\mathrm{SU}(N)$, the minima are

$$
U_{3}=\mathrm{e}^{\mathrm{i} \frac{2 \pi}{N} j} \mathbb{1}, \quad j=0, \ldots, N-1
$$

Therefore, the classical system for the $\ell_{12}=1$ sector has $N$-minima, corresponding to $N$ center broken configurations. This is analogous to our discussion on large- $T^{3} \times S_{\beta}^{1}$ where we showed that the $\ell_{12}=1$ sector was also $N$-fold degenerate, see (6.25). Of course, in quantum mechanics, center symmetry cannot break spontaneously due to tunneling effects at any finite- $N$.

How do we describe the tunneling between these minima? Let us take $U_{3}(\tau)$ of the form

$$
U_{3}=\operatorname{Diag}\left(\mathrm{e}^{\mathrm{i} \Theta_{1}}, \ldots, \mathrm{e}^{\mathrm{i} \Theta_{N}}\right), \quad \text { where } \quad \Theta_{N}=-\left(\Theta_{1}+\ldots+\Theta_{N-1}\right)
$$


Then, the (classical) potential can be expressed as

$$
V\left(\Theta_{i}\right)=-\sum_{i=1}^{N} \cos \left(\Theta_{i}-\Theta_{i+1}\right)
$$

Despite the fact that this potential looks like Gross-Pisarski-Yaffe (GPY) type potentials for gauge holonomy [124], this is superficial and they are fundamentally different. The GPY type-potentials is a quantum effect, starting at 1-loop order as $O\left(g^{2}\right)$. However, (A.7) is classical, not quantum. It is induced by the presence of classical 't Hooft flux background. As a result, for example, if one examines instantons in the theory with flux, their action is $\sim \frac{1}{g^{2}}$ since they arise from classical action. In contrast, if one examines tunneling on small $T^{3} \times \mathbb{R}$ without flux, the action is of the form $\sim \frac{1}{g}$, because these configurations arise from the balancing of classical actions against quantum induced potential, see eg. [39, 40].

To describe these tunnelings, one can take simple ansatz for $U_{3}(\tau)$

$$
U_{3}(\tau)=\operatorname{Diag}\left(\mathrm{e}^{\mathrm{i} \Theta}, \ldots, \mathrm{e}^{\mathrm{i} \Theta}, \mathrm{e}^{-\mathrm{i}(N-1) \Theta}\right)
$$

Then, the Lagrangian becomes

$$
g^{2} \mathcal{L}=(N-1) N(\mathrm{~d} \Theta)^{2}-\cos (N \Theta)
$$

where $\Theta \sim \Theta+2 \pi$. This is nothing but our $T_{N}$ model, with the distinction that the mass of the particle, instead of being set to one, is now $(N-1) N$.

The remnant of the center-symmetry (which becomes $\left(\mathbb{Z}_{N}^{[0]}\right)^{4} 0$-form symmetry on $\left.T^{3} \times S_{\beta}^{1}\right)$ is just $U_{3} \rightarrow \mathrm{e}^{\mathrm{i} \frac{2 \pi}{N}} U_{3}$ or equivalently, acting on coordinate $\Theta$ as a shift symmetry $\Theta \rightarrow \Theta+\frac{2 \pi}{N}$, i.e., the part of center-symmetry which acts on $U_{3}$ non-trivially becomes the $\mathbb{Z}_{N}$ shift symmetry of the $T_{N}$ model. To reveal the fractional instantons in the background of 't Hooft flux, we can proceed exactly as in our QM example.

We can build the fractional instanton events by considering the transition amplitudes between the minima which are $\ell_{34}$ units apart. Hamiltonian now is the one corresponding to (A.9) or more microscopically, (A.4), where $H$ already has the magnetic flux data, so we call it $H_{\ell_{12}}$. So, the quantity we are calculating is

$$
Z_{\ell_{12} \ell_{34}}=\operatorname{tr}\left[\mathrm{e}^{-\beta H_{\ell_{12}}}\left(\mathrm{U}_{\mathrm{c}}\right)^{\ell_{34}}\right]
$$

which forces twisted boundary condition in $U_{3}$ by $\ell_{34}$ unit, $U_{3}\left(\beta=L_{4}\right)=\mathrm{e}^{\mathrm{i} \frac{2 \pi}{N} \ell_{34}} U_{3}(0)$.

These fractional instantons are the ones in the theory with 't Hooft flux, $\left(\ell_{12}, \ell_{34}\right)$. They describe tunneling events in our matrix model. Roughly, $\dot{\theta} \sim E_{3}(\tau)$ and $\cos (N \theta(\tau)) \sim$ $B_{3}(\tau)$. Despite the fact that these are reasonable fractional instanton events in the $1-$ site model, they do not provide a description of the fractional instantons in continuum theory on small $T^{3} \times \mathbb{R}$ in the presence of 't Hooft flux. For once, their action is $S_{a} \sim$ $\frac{\sqrt{(N(N-1)}}{g^{2} N}$. In fact, at this stage, we are facing a problem that 't Hooft faced in a time independent continuum version of this problem [27] where the simple solutions he found did have an action which did not descend below $S_{a}=\frac{(N-1)}{N} \frac{8 \pi^{2}}{g^{2}}$. (The problem in [27] is fixed by introducing space-time dependent transition matrices.) In our case as well, this 

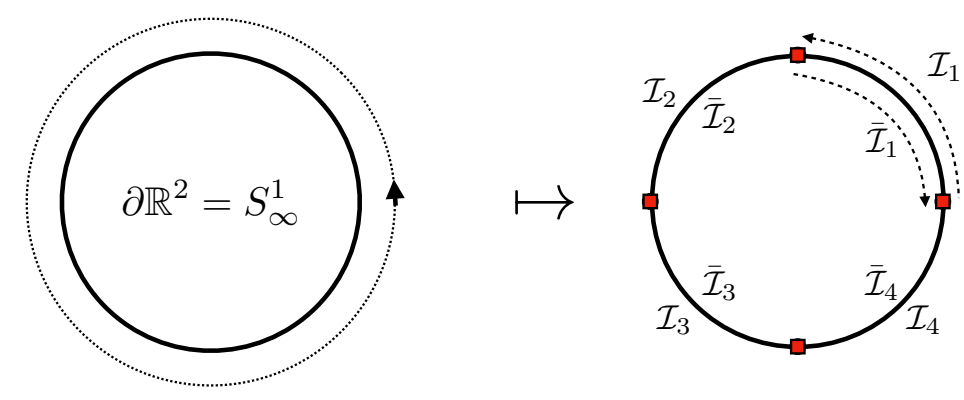

Figure 7. Mapping from $S_{\infty}^{1}$ to field space. $Q=\frac{1}{2 \pi} \oint a=\frac{1}{2 \pi} \oint \mathrm{d} \beta \in \frac{1}{N} \mathbb{Z}$, because $\mathrm{e}^{\mathrm{i} \beta(\phi)}$ is in $\mathrm{U}(1) / \mathbb{Z}_{N}$ bundle. Figure is for $\mathbb{C P}^{3}$ model.
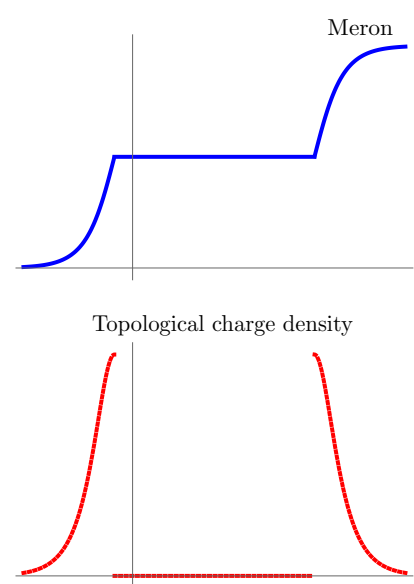

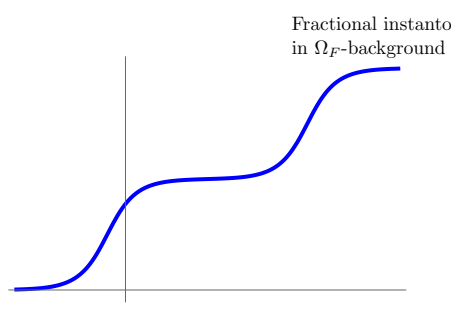

Topological charge density

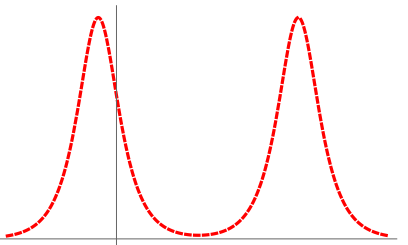

Figure 8. Horizontal axis is the angular coordinate on $S_{\infty}^{1}$ in figure 7. a) Meron data is piecewise continuous or discontinuous. Action is asserted to be log divergent [25], but that needs a reinterpretation, see text. b) Instanton solution in the $\Omega_{F}$ background. A fractional instanton pair is a critical point at infinity. Above configuration is on the Lefschetz thimble of this exact critical point. Insertion of $B^{(2)}$ 't Hooft flux, one can isolate each smooth configuration with topological charge $\frac{1}{2}$.

is an artifact of 1-site model, and the fact that we are restricting to space-independent transition functions. Our continuum formulations tells us that even at arbitrarily small volume $T^{3} \times S_{\beta}^{1}$, there exist configurations which saturate BPS bound, and this is consistent with lattice simulations on $N_{s}^{3} \times S_{\beta}^{1}$ where $N_{s} \geq 2[36,38]$.

\section{B Aside: how to fix merons in $\mathbb{C P}^{1}$ ?}

Both instanton [8] and smooth fractional instanton solutions (in the $\Omega_{F}$ or $B^{(2)}$ flux background) [11,62] have aspects in sharp contrast with meron configurations [25]. But clearly they look quite alike as shown in figure 8 , where horizontal axis is the angular coordinate on $S_{\infty}^{1}$ in figure 7 . Let us try to make some sense out of this situation for $N=2$. 
1) For merons, configurations, action densities, and topological charge densities are either piece-wise continuous or discontinuous, and they possess logarithmically divergent action [24-26].

2) Instantons are smooth single lump solutions [8]. On the other hand, the parametrization of refs. $[16,17]$, the determinant of fluctuation operator gives a precise mathematical description as if these single lumps ought to be interpreted as composites of fractional vortex instantons, despite the fact that an instanton does not physically fractionate to two pieces. Gross uses this parametrization even earlier and shows that the interaction between the instanton and anti-instanton is a dipole-dipole interaction in $2 d$ in [25]. The two are consistent.

3) The solutions in $[11,62]$ are generalization of ref. [8] to the $\Omega_{F}$ background, and it physically fractionates an instanton into pieces. If we want to single out a fractional instanton, we need to insert a $B^{(2)}$ flux, and this will guarantee a $W=1 / N$ configuration with $S=S_{I} / N$. These configurations are smooth and finite action, unlike merons.

If we superficially smoothen the meron, as suggested by Gross [25], that is close enough to exact analytic solutions in the $\Omega_{F}$ background [11,62]. What is called the logarithmically divergent action of meron pair is the logarithmic Coulomb interaction between the fractional vortex instantons. This also arise from exact computation of the determinant of the fluctuation operator $[16,17]$.

Meron idea is not generalized correctly to $\mathrm{SU}(N)$ gauge theories and $\mathbb{C P}^{N-1}$ with $N \geq 3[24-26]$. The difficulty is in the motto "meron= half-instanton". For a given $N$, the fractionalization (in center-symmetric holonomy background, or $\Omega_{F}$ or $\mathbb{Z}_{N}$ TQFT background) is always into $N$ constituents, not more, not less! Given this fact, it seems very worthwhile to revisit the idea.

't Hooft long time ago came up with a $\operatorname{PSU}(N)$ bundle construction, to figure out configurations with topological charge and action $\frac{1}{N}$. He was only able to find some exact, but uninteresting constant configurations on $T^{4}$. To be fair to him, one should state that no one was able to find non-trivial (space-time dependent) solutions in $\operatorname{PSU}(N)$ bundle up to date analytically! Numerically, they are proven to exist in $[30,31,36]$. In this work, we interpreted monopole-instanton solutions as configurations in the $\operatorname{PSU}(N)$ bundle with action $S_{I} / N$ on $\mathbb{R}^{3} \times S^{1}$ and this is a concrete realization of (another) long sought dream of 't Hooft.

't Hooft asserts that his idea of configurations with $\mathbb{Z}_{N}$ topological charge are important for the confinement and mass gap problem, but not merons or instantons [7]. But at least for $\mathrm{SU}(2)$ or $\mathbb{C P}^{1}$, these two ideas (with enough cosmetic changes) are actually the same. (I suspect both of them would think otherwise, hopefully up until today.) The correct part of the meron idea in $\mathbb{C P}^{1}$ is the "constituents" that interact with each other via long range logarithmic interactions.

Probably, the strangest insight that our work brings is following. Despite the naming "fractionalization", a physical fractionalization of action density may or may not take place. Physical fractionalization of a lump is only achieved by turning on appropriate background field. Yet, the clever parametrization of moduli space (4.3) tells us that mathematically, 
the moduli parameters acts as if they are the positions of fractional vortex instantons. This is in some sense, can be viewed as fractionalization without fractionalization.

Sometimes, it takes long time to figure out truth in a wild dream [25], and utility of a formal construction [7], perhaps, because the truth lives somewhere in between.

Open Access. This article is distributed under the terms of the Creative Commons Attribution License (CC-BY 4.0), which permits any use, distribution and reproduction in any medium, provided the original author(s) and source are credited.

\section{References}

[1] S. Gukov and A. Kapustin, Topological Quantum Field Theory, Nonlocal Operators, and Gapped Phases of Gauge Theories, arXiv:1307.4793 [INSPIRE].

[2] A. Kapustin and N. Seiberg, Coupling a QFT to a TQFT and Duality, JHEP 04 (2014) 001 [arXiv: 1401.0740] [INSPIRE].

[3] D. Gaiotto, A. Kapustin, Z. Komargodski and N. Seiberg, Theta, Time Reversal, and Temperature, JHEP 05 (2017) 091 [arXiv:1703.00501] [INSPIRE].

[4] S. Elitzur, Impossibility of Spontaneously Breaking Local Symmetries, Phys. Rev. D 12 (1975) 3978 [INSPIRE].

[5] A. Behtash, G.V. Dunne, T. Schaefer, T. Sulejmanpasic and M. Ünsal, Critical Points at Infinity, Non-Gaussian Saddles, and Bions, JHEP 06 (2018) 068 [arXiv:1803.11533] [INSPIRE].

[6] G. 't Hooft, A Property of Electric and Magnetic Flux in Nonabelian Gauge Theories, Nucl. Phys. B 153 (1979) 141 [inSPIRE].

[7] G. 't Hooft, On the Phase Transition Towards Permanent Quark Confinement, Nucl. Phys. $B 138$ (1978) 1 [INSPIRE].

[8] A.M. Polyakov and A.A. Belavin, Metastable States of Two-Dimensional Isotropic Ferromagnets, JETP Lett. 22 (1975) 245 [INSPIRE].

[9] A.A. Belavin, A.M. Polyakov, A.S. Schwartz and Y.S. Tyupkin, Pseudoparticle Solutions of the Yang-Mills Equations, Phys. Lett. B 59 (1975) 85 [INSPIRE].

[10] G.V. Dunne and M. Ünsal, New Nonperturbative Methods in Quantum Field Theory: From Large-N Orbifold Equivalence to Bions and Resurgence, Ann. Rev. Nucl. Part. Sci. 66 (2016) 245 [arXiv: 1601.03414] [inSPIRE].

[11] G.V. Dunne and M. Ünsal, Resurgence and Trans-series in Quantum Field Theory: The CP(N-1) Model, JHEP 11 (2012) 170 [arXiv:1210.2423] [INSPIRE].

[12] M. Ünsal, Magnetic bion condensation: A New mechanism of confinement and mass gap in four dimensions, Phys. Rev. D 80 (2009) 065001 [arXiv:0709.3269] [INSPIRE].

[13] M. Ünsal, Abelian duality, confinement, and chiral symmetry breaking in QCD(adj), Phys. Rev. Lett. 100 (2008) 032005 [arXiv:0708.1772] [INSPIRE].

[14] M. Ünsal and L.G. Yaffe, Center-stabilized Yang-Mills theory: Confinement and large N volume independence, Phys. Rev. D 78 (2008) 065035 [arXiv:0803.0344] [INSPIRE]. 
[15] M. Shifman and M. Ünsal, QCD-like Theories on $R_{3} \times S_{1}$ : A Smooth Journey from Small to Large $r\left(S_{1}\right)$ with Double-Trace Deformations, Phys. Rev. D 78 (2008) 065004 [arXiv:0802.1232] [INSPIRE].

[16] B. Berg and M. Lüscher, Computation of Quantum Fluctuations Around Multi-Instanton Fields from Exact Green's Functions: The $C P^{n-1}$ Case, Commun. Math. Phys. 69 (1979) 57 [INSPIRE].

[17] V.A. Fateev, I.V. Frolov and A.S. Shvarts, Quantum Fluctuations of Instantons in the Nonlinear Sigma Model, Nucl. Phys. B 154 (1979) 1 [INSPIRE].

[18] J.M. Kosterlitz, The Critical properties of the two-dimensional x y model, J. Phys. $C \mathbf{7}$ (1974) 1046 [INSPIRE].

[19] J.M. Kosterlitz and D.J. Thouless, Ordering, metastability and phase transitions in two-dimensional systems, J. Phys. C 6 (1973) 1181 [INSPIRE].

[20] S. Coleman, Aspects of Symmetry: Selected Erice Lectures, Cambridge University Press, Cambridge U.K. (1988).

[21] A.I. Vainshtein, V.I. Zakharov, V.A. Novikov and M.A. Shifman, ABC's of Instantons, Sov. Phys. Usp. 25 (1982) 195 [INSPIRE].

[22] T. Schäfer and E.V. Shuryak, Instantons in QCD, Rev. Mod. Phys. 70 (1998) 323 [hep-ph/9610451] [INSPIRE].

[23] M. Mariño, Instantons and Large N: An Introduction to Non-Perturbative Methods in Quantum Field Theory, Cambridge University Press, Cambridge U.K. (2015).

[24] C.G. Callan, Jr., R.F. Dashen and D.J. Gross, Toward a Theory of the Strong Interactions, Phys. Rev. D 17 (1978) 2717 [inSPIRE].

[25] D.J. Gross, Meron Configurations in the Two-Dimensional O(3) Sigma Model, Nucl. Phys. B 132 (1978) 439 [INSPIRE].

[26] C.G. Callan, Jr., R.F. Dashen and D.J. Gross, A Mechanism for Quark Confinement, Phys. Lett. B 66 (1977) 375 [inSPIRE].

[27] G. 't Hooft, Some Twisted Selfdual Solutions for the Yang-Mills Equations on a Hypertorus, Commun. Math. Phys. 81 (1981) 267 [InSPIRE].

[28] P. van Baal, Some Results for SU(N) Gauge Fields on the Hypertorus, Commun. Math. Phys. 85 (1982) 529 [INSPIRE].

[29] E. Witten, Constraints on Supersymmetry Breaking, Nucl. Phys. B 202 (1982) 253 [INSPIRE].

[30] M. Garcia Perez, A. Gonzalez-Arroyo and B. Soderberg, Minimum Action Solutions for SU(2) Gauge Theory on the Torus With Nonorthogonal Twist, Phys. Lett. B 235 (1990) 117 [INSPIRE].

[31] M. Garcia Perez and A. Gonzalez-Arroyo, Numerical study of Yang-Mills classical solutions on the twisted torus, J. Phys. A 26 (1993) 2667 [hep-lat/9206016] [INSPIRE].

[32] M. Garcia Perez, A. Gonzalez-Arroyo and P. Martinez, From perturbation theory to confinement: How the string tension is built up, Nucl. Phys. B Proc. Suppl. 34 (1994) 228 [hep-lat/9312066] [INSPIRE].

[33] A. Gonzalez-Arroyo and P. Martinez, Investigating Yang-Mills theory and confinement as a function of the spatial volume, Nucl. Phys. B 459 (1996) 337 [hep-lat/9507001] [INSPIRE]. 
[34] A. Gonzalez-Arroyo, P. Martinez and A. Montero, Gauge invariant structures and confinement, Phys. Lett. B 359 (1995) 159 [hep-lat/9507006] [INSPIRE].

[35] A. Gonzalez-Arroyo and A. Montero, Do classical configurations produce confinement?, Phys. Lett. B 387 (1996) 823 [hep-th/9604017] [INSPIRE].

[36] A. Montero, Numerical analysis of fractional charge solutions on the torus, JHEP 05 (2000) 022 [hep-lat/0004009] [INSPIRE].

[37] A. Gonzalez-Arroyo, Yang-Mills fields on the four-dimensional torus. Part 1. Classical theory, in Advanced Summer School on Nonperturbative Quantum Field Physics, Peniscola Spain (1977), pg. 57 [hep-th/9807108] [INSPIRE].

[38] A. González-Arroyo, Constructing SU(N) fractional instantons, JHEP 02 (2020) 137 [arXiv: 1910.12565] [INSPIRE].

[39] M. Lüscher, Some Analytic Results Concerning the Mass Spectrum of Yang-Mills Gauge Theories on a Torus, Nucl. Phys. B 219 (1983) 233 [InSPIRE].

[40] P. van Baal and J. Koller, QCD on a Torus and Electric Flux Energies From Tunneling, Annals Phys. 174 (1987) 299 [INSPIRE].

[41] P. van Baal, QCD in a finite volume, hep-ph/0008206 [InSPIRE].

[42] T. Eguchi and H. Kawai, Reduction of Dynamical Degrees of Freedom in the Large $N$ Gauge Theory, Phys. Rev. Lett. 48 (1982) 1063 [InSPIRE].

[43] G. Bhanot, U.M. Heller and H. Neuberger, The Quenched Eguchi-Kawai Model, Phys. Lett. B 113 (1982) 47 [INSPIRE].

[44] J. Kiskis, R. Narayanan and H. Neuberger, Does the crossover from perturbative to nonperturbative physics in $Q C D$ become a phase transition at infinite N?, Phys. Lett. B $\mathbf{5 7 4}$ (2003) 65 [hep-lat/0308033] [INSPIRE].

[45] A. Gonzalez-Arroyo and M. Okawa, Large $N$ reduction with the Twisted Eguchi-Kawai model, JHEP 07 (2010) 043 [arXiv: 1005.1981] [INSPIRE].

[46] E. Witten, Large N Chiral Dynamics, Annals Phys. 128 (1980) 363 [INSPIRE].

[47] M. Ünsal, TQFT at work for IR-renormalons, resurgence and Lefschetz decomposition, arXiv:2106.14971 [INSPIRE].

[48] M. Ünsal, Theta dependence, sign problems and topological interference, Phys. Rev. D 86 (2012) 105012 [arXiv:1201.6426] [INSPIRE].

[49] Y. Kikuchi and Y. Tanizaki, Global inconsistency, 't Hooft anomaly, and level crossing in quantum mechanics, PTEP 2017 (2017) 113B05 [arXiv:1708.01962] [INSPIRE].

[50] A. Cherman, D. Dorigoni and M. Ünsal, Decoding perturbation theory using resurgence: Stokes phenomena, new saddle points and Lefschetz thimbles, JHEP 10 (2015) 056 [arXiv: 1403.1277] [INSPIRE].

[51] J. Zinn-Justin, Quantum field theory and critical phenomena, Int. Ser. Monogr. Phys. 113 (2002) 1.

[52] Y. Tanizaki, T. Misumi and N. Sakai, Circle compactification and 't Hooft anomaly, JHEP 12 (2017) 056 [arXiv: 1710.08923] [INSPIRE]. 
[53] T. Fujimori, S. Kamata, T. Misumi, M. Nitta and N. Sakai, Exact resurgent trans-series and multibion contributions to all orders, Phys. Rev. D 95 (2017) 105001 [arXiv: 1702.00589] [INSPIRE].

[54] T. Fujimori, S. Kamata, T. Misumi, M. Nitta and N. Sakai, Bion non-perturbative contributions versus infrared renormalons in two-dimensional $\mathbb{C} P^{N-1}$ models, JHEP 02 (2019) 190 [arXiv:1810.03768] [INSPIRE].

[55] K. Ishikawa, O. Morikawa, A. Nakayama, K. Shibata, H. Suzuki and H. Takaura, Infrared renormalon in the supersymmetric $\mathbb{C} P^{N-1}$ model on $\mathbb{R} \times S^{1}$, PTEP 2020 (2020) 023B10 [arXiv: 1908.00373] [INSPIRE].

[56] T. Misumi, M. Nitta and N. Sakai, Neutral bions in the $\mathbb{C} P^{N-1}$ model, JHEP 06 (2014) 164 [arXiv: 1404.7225] [INSPIRE].

[57] T. Misumi, T. Fujimori, E. Itou, M. Nitta and N. Sakai, Lattice study on the twisted $\mathbb{C} P^{N-1}$ models on $\mathbb{R} \times S^{1}$, PoS LATTICE2019 (2019) 015 [arXiv: 1911.07398] [INSPIRE].

[58] M. Eto, Y. Isozumi, M. Nitta, K. Ohashi and N. Sakai, Instantons in the Higgs phase, Phys. Rev. D 72 (2005) 025011 [hep-th/0412048] [INSPIRE].

[59] M. Eto, Y. Isozumi, M. Nitta, K. Ohashi and N. Sakai, Solitons in the Higgs phase: The Moduli matrix approach, J. Phys. A 39 (2006) R315 [hep-th/0602170] [InSPIRE].

[60] M. Eto et al., Non-Abelian vortices on cylinder: Duality between vortices and walls, Phys. Rev. D 73 (2006) 085008 [hep-th/0601181] [INSPIRE].

[61] W. Brendel, F. Bruckmann, L. Janssen, A. Wipf and C. Wozar, Instanton constituents and fermionic zero modes in twisted $C P^{n}$ models, Phys. Lett. B 676 (2009) 116 [arXiv: 0902 .2328] [INSPIRE].

[62] F. Bruckmann, Instanton constituents in the $O(3)$ model at finite temperature, Phys. Rev. Lett. 100 (2008) 051602 [arXiv:0707.0775] [INSPIRE].

[63] E. Witten, Instantons, the Quark Model, and the 1/n Expansion, Nucl. Phys. B 149 (1979) 285 [INSPIRE].

[64] G.V. Dunne, Y. Tanizaki and M. Ünsal, Quantum Distillation of Hilbert Spaces, Semi-classics and Anomaly Matching, JHEP 08 (2018) 068 [arXiv:1803.02430] [INSPIRE].

[65] A. Cherman, S. Sen, M. Ünsal, M.L. Wagman and L.G. Yaffe, Order parameters and color-flavor center symmetry in QCD, Phys. Rev. Lett. 119 (2017) 222001 [arXiv: 1706.05385] [INSPIRE].

[66] A. Polyakov, Mathematical Reports. Vol. 3: Gauge Fields and Strings, first edition, CRC Press, Boca Raton U.S.A. (1987).

[67] T. Sulejmanpasic, Global Symmetries, Volume Independence, and Continuity in Quantum Field Theories, Phys. Rev. Lett. 118 (2017) 011601 [arXiv:1610.04009] [InSPIRE].

[68] A. D'Adda, M. Lüscher and P. Di Vecchia, A 1/n Expandable Series of Nonlinear Sigma Models with Instantons, Nucl. Phys. B 146 (1978) 63 [InSPIRE].

[69] E. Vicari and H. Panagopoulos, Theta dependence of $\mathrm{SU}(N)$ gauge theories in the presence of a topological term, Phys. Rept. 470 (2009) 93 [arXiv:0803.1593] [InSPIRE].

[70] A.M. Polyakov, Compact Gauge Fields and the Infrared Catastrophe, Phys. Lett. B 59 (1975) 82 [INSPIRE]. 
[71] S.R. Coleman, More About the Massive Schwinger Model, Annals Phys. 101 (1976) 239 [INSPIRE].

[72] G. 't Hooft, Can We Make Sense Out of Quantum Chromodynamics?, Subnucl. Ser. 15 (1979) 943 [INSPIRE].

[73] G. Munster, On the Statistical Mechanics of Dense Instanton Gases, Z. Phys. C 12 (1982) 43 [INSPIRE].

[74] A. Bukhvostov and L. Lipatov, Instanton-Anti-instanton Interaction in the O(3) Nonlinear $\Sigma$ Model and an Exactly Soluble Fermion Theory, Pisma Zh. Eksp. Teor. Fiz. 31 (1980) 138.

[75] T. Misumi, Y. Tanizaki and M. Ünsal, Fractional $\theta$ angle, 't Hooft anomaly, and quantum instantons in charge-q multi-flavor Schwinger model, JHEP 07 (2019) 018 [arXiv: 1905. 05781] [INSPIRE].

[76] S.R. Coleman, The Quantum sine-Gordon Equation as the Massive Thirring Model, Phys. Rev. D 11 (1975) 2088 [INSPIRE].

[77] A.V. Smilga, On the fermion condensate in Schwinger model, Phys. Lett. B 278 (1992) 371 [INSPIRE].

[78] J.E. Hetrick, Y. Hosotani and S. Iso, The Massive multi-flavor Schwinger model, Phys. Lett. $B 350$ (1995) 92 [hep-th/9502113] [INSPIRE].

[79] Y. Hosotani and R. Rodriguez, Bosonized massive N flavor Schwinger model, J. Phys. A 31 (1998) 9925 [hep-th/9804205] [INSPIRE].

[80] K. Wamer and I. Affleck, Mass generation by fractional instantons in $\mathrm{SU}(n)$ chains, Phys. Rev. B 101 (2020) 245143 [arXiv: 2001.09114] [INSPIRE].

[81] Y. Tanizaki and T. Sulejmanpasic, Anomaly and global inconsistency matching: $\theta$-angles, $\mathrm{SU}(3) / \mathrm{U}(1)^{2}$ nonlinear sigma model, $\mathrm{SU}(3)$ chains and its generalizations, Phys. Rev. $B \mathbf{9 8}$ (2018) 115126 [arXiv:1805.11423] [InSPIRE].

[82] E. Witten, Nonabelian Bosonization in Two-Dimensions, Commun. Math. Phys. 92 (1984) 455 [INSPIRE].

[83] A.M. Polyakov and P.B. Wiegmann, Theory of Nonabelian Goldstone Bosons, Phys. Lett. B 131 (1983) 121 [INSPIRE].

[84] A.M. Polyakov and P.B. Wiegmann, Goldstone Fields in Two-Dimensions with Multivalued Actions, Phys. Lett. B 141 (1984) 223 [INSPIRE].

[85] A. Cherman and M. Ünsal, Critical behavior of gauge theories and Coulomb gases in three and four dimensions, arXiv:1711.10567 [INSPIRE].

[86] E. Fradkin, Field Theories of Condensed Matter Physics, second edition, Cambridge University Press, Cambridge U.K. (2013).

[87] F.D.M. Haldane, Nonlinear field theory of large spin Heisenberg antiferromagnets. Semiclassically quantized solitons of the one-dimensional easy Axis Neel state, Phys. Rev. Lett. 50 (1983) 1153 [INSPIRE].

[88] I. Affleck and F.D.M. Haldane, Critical Theory of Quantum Spin Chains, Phys. Rev. B 36 (1987) 5291 [INSPIRE].

[89] A. Behtash, T. Sulejmanpasic, T. Schäfer and M. Ünsal, Hidden topological angles and Lefschetz thimbles, Phys. Rev. Lett. 115 (2015) 041601 [arXiv: 1502.06624] [INSPIRE]. 
[90] I. Affleck, Critical Behavior of SU(n) Quantum Chains and Topological Nonlinear $\sigma$ Models, Nucl. Phys. B 305 (1988) 582 [INSPIRE].

[91] E. Poppitz and M. Ünsal, Seiberg-Witten and 'Polyakov-like' magnetic bion confinements are continuously connected, JHEP 07 (2011) 082 [arXiv:1105.3969] [INSPIRE].

[92] E. Poppitz, T. Schäfer and M. Ünsal, Continuity, Deconfinement, and (Super) Yang-Mills Theory, JHEP 10 (2012) 115 [arXiv:1205.0290] [INSPIRE].

[93] E. Poppitz, T. Schäfer and M. Ünsal, Universal mechanism of (semi-classical) deconfinement and theta-dependence for all simple groups, JHEP 03 (2013) 087 [arXiv: 1212.1238] [INSPIRE].

[94] P. Argyres and M. Ünsal, A semiclassical realization of infrared renormalons, Phys. Rev. Lett. 109 (2012) 121601 [arXiv:1204.1661] [INSPIRE].

[95] P.C. Argyres and M. Ünsal, The semi-classical expansion and resurgence in gauge theories: new perturbative, instanton, bion, and renormalon effects, JHEP 08 (2012) 063 [arXiv: 1206.1890] [INSPIRE].

[96] M.M. Anber, E. Poppitz and M. Ünsal, $2 d$ affine XY-spin model/4d gauge theory duality and deconfinement, JHEP 04 (2012) 040 [arXiv:1112.6389] [INSPIRE].

[97] M.M. Anber and E. Poppitz, On the global structure of deformed Yang-Mills theory and $Q C D(a d j)$ on $\mathbb{R}^{3} \times \mathbb{S}^{1}, J H E P 10(2015) 051$ [arXiv: 1508.00910] [INSPIRE].

[98] T. Misumi and T. Kanazawa, Adjoint $Q C D$ on $\mathbb{R}^{3} \times S^{1}$ with twisted fermionic boundary conditions, JHEP 06 (2014) 181 [arXiv:1405.3113] [INSPIRE].

[99] E. Poppitz and M. Ünsal, Index theorem for topological excitations on $R^{3} \times S^{1}$ and Chern-Simons theory, JHEP 03 (2009) 027 [arXiv: 0812.2085] [INSPIRE].

[100] K. Aitken, A. Cherman, E. Poppitz and L.G. Yaffe, QCD on a small circle, Phys. Rev. D 96 (2017) 096022 [arXiv: 1707.08971] [INSPIRE].

[101] E. Thomas and A.R. Zhitnitsky, Topological Susceptibility and Contact Term in QCD. A Toy Model, Phys. Rev. D 85 (2012) 044039 [arXiv:1109.2608] [INSPIRE].

[102] A.R. Zhitnitsky, QCD as a topologically ordered system, Annals Phys. 336 (2013) 462 [arXiv:1301.7072] [INSPIRE].

[103] M.M. Anber and A.R. Zhitnitsky, Oblique Confinement at $\theta \neq 0$ in weakly coupled gauge theories with deformations, Phys. Rev. D 96 (2017) 074022 [arXiv:1708.07520] [InSPIRE].

[104] E. Itou, Resurgence and fractional instanton of the SU(3) gauge theory in weak coupling regime, PoS LATTICE2019 (2019) 014 [arXiv: 1910.12425] [INSPIRE].

[105] M.M. Anber, $\Theta$ dependence of the deconfining phase transition in pure $\mathrm{SU}\left(N_{c}\right)$ Yang-Mills theories, Phys. Rev. D 88 (2013) 085003 [arXiv: 1302.2641] [InSPIRE].

[106] R. Kitano, T. Suyama and N. Yamada, $\theta=\pi$ in $\mathrm{SU}(N) / \mathbb{Z}_{N}$ gauge theories, JHEP 09 (2017) 137 [arXiv: 1709. 04225] [inSPIRE].

[107] N. Kan, R. Kitano, S. Yankielowicz and R. Yokokura, From 3d dualities to hadron physics, Phys. Rev. D 102 (2020) 125034 [arXiv:1909.04082] [INSPIRE].

[108] Y. Liu, E. Shuryak and I. Zahed, Light quarks in the screened dyon-antidyon Coulomb liquid model. II, Phys. Rev. D 92 (2015) 085007 [arXiv: 1503.09148] [InSPIRE]. 
[109] Y. Liu, E. Shuryak and I. Zahed, Confining dyon-antidyon Coulomb liquid model. I, Phys. Rev. D 92 (2015) 085006 [arXiv: 1503.03058] [INSPIRE].

[110] R.N. Larsen, S. Sharma and E. Shuryak, The topological objects near the chiral crossover transition in QCD, Phys. Lett. B $\mathbf{7 9 4}$ (2019) 14 [arXiv:1811.07914] [INSPIRE].

[111] C. Bonati, M. Cardinali and M. D'Elia, $\theta$ dependence in trace deformed SU(3) Yang-Mills theory: a lattice study, Phys. Rev. D 98 (2018) 054508 [arXiv:1807.06558] [InSPIRE].

[112] C. Bonati, M. Cardinali, M. D'Elia and F. Mazziotti, $\theta$-dependence and center symmetry in Yang-Mills theories, Phys. Rev. D 101 (2020) 034508 [arXiv:1912.02662] [inSPIRE].

[113] A.R. Zhitnitsky, Once more on theta vacua in $(2+1)$-dimensional QED and (3+1)-dimensional gluodynamics, Phys. Lett. B 291 (1992) 465 [hep-ph/9207250] [INSPIRE].

[114] F. Bruckmann, D. Nogradi and P. van Baal, Constituent monopoles through the eyes of fermion zero modes, Nucl. Phys. B 6666 (2003) 197 [hep-th/0305063] [INSPIRE].

[115] M. Garcia Perez, A. Gonzalez-Arroyo, C. Pena and P. van Baal, Nahm dualities on the torus: A Synthesis, Nucl. Phys. B 564 (2000) 159 [hep-th/9905138] [INSPIRE].

[116] M. Garcia Perez, A. Gonzalez-Arroyo and A. Sastre, Adjoint zero-modes as a tool to understand the Yang-Mills vacuum, PoS LATTICE2007 (2007) 328 [arXiv:0710.0455] [INSPIRE].

[117] M. Garcia Perez, A. Gonzalez-Arroyo and A. Sastre, From confinement to adjoint zero-modes, eCONF C 0906083 (2009) 06 [arXiv: 1003.5022] [INSPIRE].

[118] RTN collaboration, Instanton like contributions to the dynamics of Yang-Mills fields on the twisted torus, Phys. Lett. B 305 (1993) 366 [hep-lat/9302007] [INSPIRE].

[119] D.R. Lebedev, M.I. Polikarpov and A.A. Roslyi, Gauge Fields on the Continuum and Lattice Tori, Nucl. Phys. B 325 (1989) 138 [INSPIRE].

[120] M. Garcia Perez, A. Gonzalez-Arroyo, A. Montero and P. van Baal, Calorons on the lattice: A New perspective, JHEP 06 (1999) 001 [hep-lat/9903022] [INSPIRE].

[121] E. Witten, Supersymmetric index in four-dimensional gauge theories, Adv. Theor. Math. Phys. 5 (2002) 841 [hep-th/0006010] [INSPIRE].

[122] E. Cohen and C. Gomez, Chiral Symmetry Breaking in Supersymmetric Yang-Mills, Phys. Rev. Lett. 52 (1984) 237 [inSPIRE].

[123] T. Sulejmanpasic, Y. Tanizaki and M. Ünsal, Universality between vector-like and chiral quiver gauge theories: Anomalies and domain walls, JHEP 06 (2020) 173 [arXiv:2004.10328] [INSPIRE].

[124] D.J. Gross, R.D. Pisarski and L.G. Yaffe, $Q C D$ and Instantons at Finite Temperature, Rev. Mod. Phys. 53 (1981) 43 [inSPIRE].

[125] K.-M. Lee and P. Yi, Monopoles and instantons on partially compactified D-branes, Phys. Rev. D 56 (1997) 3711 [hep-th/9702107] [INSPIRE].

[126] K.-M. Lee and C.-H. Lü, SU(2) calorons and magnetic monopoles, Phys. Rev. D 58 (1998) 025011 [hep-th/9802108] [INSPIRE].

[127] T.C. Kraan and P. van Baal, Exact T duality between calorons and Taub-NUT spaces, Phys. Lett. B 428 (1998) 268 [hep-th/9802049] [INSPIRE]. 
[128] T.C. Kraan and P. van Baal, Periodic instantons with nontrivial holonomy, Nucl. Phys. B 533 (1998) 627 [hep-th/9805168] [INSPIRE].

[129] T.C. Kraan and P. van Baal, Monopole constituents inside $\mathrm{SU}(N)$ calorons, Phys. Lett. B 435 (1998) 389 [hep-th/9806034] [INSPIRE].

[130] B. Collie and D. Tong, The Partonic Nature of Instantons, JHEP 08 (2009) 006 [arXiv: 0905.2267] [INSPIRE].

[131] N.M. Davies, T.J. Hollowood and V.V. Khoze, Monopoles, affine algebras and the gluino condensate, J. Math. Phys. 44 (2003) 3640 [hep-th/0006011] [INSPIRE].

[132] Y. Tanizaki and M. Ünsal, Modified instanton sum in QCD and higher-groups, JHEP 03 (2020) 123 [arXiv: 1912.01033] [INSPIRE].

[133] D. Diakonov and V. Petrov, Confining ensemble of dyons, Phys. Rev. D 76 (2007) 056001 [arXiv: 0704.3181] [INSPIRE].

[134] D. Diakonov and V. Petrov, Confinement and deconfinement for any gauge group from dyons viewpoint, AIP Conf. Proc. 1343 (2011) 69 [arXiv:1011.5636] [InSPIRE].

[135] K. Aitken, A. Cherman and M. Ünsal, Vacuum structure of Yang-Mills theory as a function of $\theta$, JHEP 09 (2018) 030 [arXiv: 1804.06848] [INSPIRE].

[136] D. Gaiotto, A. Kapustin, N. Seiberg and B. Willett, Generalized Global Symmetries, JHEP 02 (2015) 172 [arXiv:1412.5148] [INSPIRE].

[137] Z. Wan, J. Wang and Y. Zheng, Quantum 4d Yang-Mills Theory and Time-Reversal Symmetric 5d Higher-Gauge Topological Field Theory, Phys. Rev. D 100 (2019) 085012 [arXiv: 1904.00994] [INSPIRE].

[138] J. Greensite, The Confinement problem in lattice gauge theory, Prog. Part. Nucl. Phys. 51 (2003) 1 [hep-lat/0301023] [INSPIRE].

[139] T. Banks, Modern Quantum Field Theory: A Concise Introduction, Cambridge University Press, Cambridge U.K. (2014).

[140] A. Cherman and E. Poppitz, Emergent dimensions and branes from large- $N$ confinement, Phys. Rev. D 94 (2016) 125008 [arXiv:1606.01902] [InSPIRE].

[141] M. Yamazaki and K. Yonekura, From $4 d$ Yang-Mills to $2 d \mathbb{C P}^{N-1}$ model: IR problem and confinement at weak coupling, JHEP 07 (2017) 088 [arXiv:1704.05852] [INSPIRE].

[142] M. Yamazaki, Relating 't Hooft Anomalies of $4 d$ Pure Yang-Mills and $2 d \mathbb{C P}^{N-1}$ Model, JHEP 10 (2018) 172 [arXiv:1711.04360] [INSPIRE].

[143] Z. Wan, J. Wang and Y. Zheng, New higher anomalies, SU(N) Yang-Mills gauge theory and $\mathbb{C P}^{\mathrm{N}-1}$ sigma model, Annals Phys. 414 (2020) 168074 [arXiv: 1812.11968] [INSPIRE].

[144] G. 't Hooft, Magnetic Monopoles in Unified Gauge Theories, Nucl. Phys. B 79 (1974) 276 [INSPIRE].

[145] A.M. Polyakov, Particle Spectrum in Quantum Field Theory, JETP Lett. 20 (1974) 194 [INSPIRE].

[146] J.S. Bell and R. Jackiw, A PCAC puzzle: $\pi^{0} \rightarrow \gamma \gamma$ in the $\sigma$ model, Nuovo Cim. A 60 (1969) 47 [INSPIRE].

[147] A. Roberge and N. Weiss, Gauge Theories With Imaginary Chemical Potential and the Phases of QCD, Nucl. Phys. B 275 (1986) 734 [inSPIRE]. 
[148] N. Seiberg and E. Witten, Electric-magnetic duality, monopole condensation, and confinement in $N=2$ supersymmetric Yang-Mills theory, Nucl. Phys. B 426 (1994) 19 [Erratum ibid. 430 (1994) 485] [hep-th/9407087] [INSPIRE].

[149] M.R. Douglas and S.H. Shenker, Dynamics of SU(N) supersymmetric gauge theory, Nucl. Phys. B 447 (1995) 271 [hep-th/9503163] [INSPIRE].

[150] E. Poppitz and M.E. Shalchian T., String tensions in deformed Yang-Mills theory, JHEP 01 (2018) 029 [arXiv:1708.08821] [InSPIRE].

[151] J. Greensite, Confinement from Center Vortices: A review of old and new results, EPJ Web Conf. 137 (2017) 01009 [arXiv: 1610.06221] [INSPIRE].

[152] O. Aharony, A. Hanany, K.A. Intriligator, N. Seiberg and M.J. Strassler, Aspects of $N=2$ supersymmetric gauge theories in three-dimensions, Nucl. Phys. B 499 (1997) 67 [hep-th/9703110] [INSPIRE].

[153] M. Engelhardt, Center vortex model for the infrared sector of SU(3) Yang-Mills theory: Topological susceptibility, Phys. Rev. D 83 (2011) 025015 [arXiv: 1008.4953] [InSPIRE].

[154] D. Trewartha, W. Kamleh and D. Leinweber, Connection between center vortices and instantons through gauge-field smoothing, Phys. Rev. D 92 (2015) 074507 [arXiv: 1509.05518] [INSPIRE].

[155] K.G. Wilson, Confinement of Quarks, Phys. Rev. D 10 (1974) 2445 [inSPIRE].

[156] C.P. Bachas and R.F. Dashen, Phase Transitions and Local Minima of the Action, Nucl. Phys. B 210 (1982) 583 [INSPIRE].

[157] A. Cherman, T. Schäfer and M. Ünsal, Chiral Lagrangian from Duality and Monopole Operators in Compactified QCD, Phys. Rev. Lett. 117 (2016) 081601 [arXiv:1604.06108] [INSPIRE].

[158] A. Gonzalez-Arroyo and M. Okawa, A Twisted Model for Large N Lattice Gauge Theory, Phys. Lett. B 120 (1983) 174 [INSPIRE].

[159] A. Gonzalez-Arroyo and M. Okawa, The Twisted Eguchi-Kawai Model: A Reduced Model for Large N Lattice Gauge Theory, Phys. Rev. D 27 (1983) 2397 [InSPIRE]. 